JOURNAL OF

SYMPLECTIC GEOMETRY

Volume 6, Number 3, 267-351, 2008

\title{
FIBERED SYMPLECTIC COHOMOLOGY AND THE LERAY-SERRE SPECTRAL SEQUENCE
}

\author{
AleXANDRu OANCEA
}

We define symplectic cohomology groups $F H_{[a, b]}^{*}(E),-\infty \leq a<$ $b \leq \infty$ for a class of symplectic fibrations $F \hookrightarrow E \longrightarrow B$ with closed symplectic base and convex at infinity fiber. The crucial geometric assumption on the fibration is a negativity property reminiscent of negative curvature in complex vector bundles. When $B$ is symplectically aspherical, we construct a spectral sequence of Leray-Serre type converging to $F H_{[a, b]}^{*}(E)$, and we use it to prove new cases of the Weinstein conjecture.

\section{Contents}

1. Introduction 267

2. Negative symplectic fibrations 273

3. Examples $\quad 277$

4. Fibered symplectic cohomology groups 285

5. Pseudo-gradient vector fields 307

6. Transversality for split almost complex structures 319

$\begin{array}{ll}\text { 7. The spectral sequence } & 329\end{array}$

8. Appendix A. On Symplectic Forms and the Taming Property 348

$\begin{array}{ll}\text { References } & 349\end{array}$

\section{Introduction}

This paper investigates the behavior of symplectic cohomology in a fibered context. The foundational papers on symplectic (co)homology are $[\mathbf{6}, \mathbf{1 1}$, 31], and recent developments are presented in [29]. The symplectic fibrations $F \hookrightarrow E \stackrel{\pi}{\longrightarrow} B$ that we consider have a closed symplectic base $(B, \beta)$, a fiber with contact type boundary and possess a coupling form $\Omega$. In this 
sense, they are very close to being Hamiltonian [22]. Moreover, they must satisfy a negativity property reminiscent of negative curvature in Hermitian vector bundles, as well as a monodromy condition allowing to define parallel transport along the boundary. We call them in the sequel negative symplectic fibrations (see Definition 2.1). The total space $E$ is a symplectic manifold with symplectic form $\omega_{\epsilon}=\pi^{*} \beta+\epsilon \Omega, \epsilon>0$ but the boundary is not necessarily of contact type. It only satisfies a fiberwise convexity condition which is nevertheless sufficient for us to define symplectic homology and cohomology groups $F H_{*}^{[a, b]}(E)$ and $F H_{[a, b]}^{*}(E),-\infty \leq a<b \leq+\infty$. Our definition is a fibered extension of the one of Viterbo [31] — which corresponds to the case $B=\{$ pt. $\}$ - and features the same functorial properties.

We call a symplectic manifold $(B, \beta)$ symplectically aspherical if we have $\int f^{*} \beta=0$ for any smooth map $f: \mathbb{S}^{2} \longrightarrow B$. We say that $(B, \beta)$ is monotone if there exists $\lambda \geq 0$ such that $\langle[\beta],[f]\rangle=\lambda\left\langle c_{1}(T B),[f]\right\rangle$ for any such $f$. Our main result is the following.

Theorem A. Let $F \hookrightarrow E \rightarrow B$ be a negative symplectic fibration, and assume $B$ to be symplectically aspherical and $E$ monotone. For any field of coefficients and any choice of real numbers $-\infty \leq a<b \leq \infty$, there exists a cohomology spectral sequence $E_{r}^{p, q}(a, b) \Longrightarrow F H_{[a, b]}^{*}(E), r \geq 2$ such that

$$
E_{2}^{p, q}(a, b) \simeq H^{n+p}\left(B ; \mathcal{F} \mathcal{H}_{[a, b]}^{q}(F)\right), \quad n=\frac{1}{2} \operatorname{dim} B .
$$

The spectral sequence is canonical and the notation $\mathcal{F H}_{[a, b]}^{*}(F)$ stands for a local system of coefficients with fiber $F H_{[a, b]}^{*}(F)$. The spectral sequence is compatible with the truncation morphisms.

The "truncation" morphisms in the statement are canonical morphisms

$$
F H_{[a, b]}^{*}(E) \longrightarrow F H_{\left[a^{\prime}, b^{\prime}\right]}^{*}(E), \quad a \geq a^{\prime}, b \geq b^{\prime}
$$

induced by the truncation of the range of the Hamiltonian action.

Let

$$
\nu=\min \left\{\left|\left\langle f^{*} c_{1}(T E),\left[\mathbb{S}^{2}\right]\right\rangle\right|: f: \mathbb{S}^{2} \longrightarrow E\right\}
$$

be the minimal Chern number of $E$. The first Chern class of $T E$ is computed with respect to an almost complex structure which is compatible with the symplectic form $\omega_{\epsilon}$. If $\nu \neq 0$, the Floer cohomology groups are only $\mathbb{Z} / 2 \nu \mathbb{Z}$ graded and the statement should be understood modulo $2 \nu$. We shall not mention anymore this grading issue in the course of the paper because it is of a purely formal nature and has no bearing on the flow of the arguments.

The cohomology spectral sequence is constructed only with field coefficients because the symplectic cohomology groups are defined as an inverse limit. We need in the proof of Theorem A that the inverse limit functor be exact, which is true if the terms involved in the limit are finite dimensional vector spaces $([\mathbf{8}]$, Chapter VIII). On the other hand, the dual homology 
spectral sequence exists with arbitrary coefficients because the symplectic homology groups are defined as a direct limit, which is an exact functor (see Remark 4.28). Unless otherwise mentioned, we will use from now on field coefficients whenever symplectic cohomology groups are involved.

Let us now introduce the following definition. Given a symplectically aspherical manifold $(M, \omega)$ with boundary of contact type, we say that $\partial M$ is of positive contact type if every positively oriented closed contractible characteristic $\gamma$ has positive action $A_{\omega}(\gamma):=\int_{D^{2}} \bar{\gamma}^{*} \omega$ bounded away from zero. Here $\bar{\gamma}: D^{2} \rightarrow M$ is any smooth extension of $\gamma: \mathbb{S}^{1} \rightarrow M$ (see Section 4.3 for a discussion of this notion). Our main class of examples is that of convex exact symplectic manifolds.

We recall that the homological structure of the fibration $(E, \pi, B, F)$ is captured by the Leray-Serre spectral sequence ${ }_{\text {LS }} E_{r}^{p, q} \Rightarrow H^{p+q}(E, \partial E)$ with ${ }_{\mathrm{LS}} E_{2}^{p, q} \simeq H^{p}\left(B ; \mathcal{H}^{q}(F, \partial F)\right)$, where $\mathcal{H}^{q}(F, \partial F)$ is the local system of coefficients on $B$ given by the locally constant presheaf $U \mapsto H^{q}\left(\pi^{-1}(U), \pi^{-1}(U) \cap\right.$ $\partial E)$.

Theorem B. Let $F \hookrightarrow E \rightarrow B$ be a negative symplectic fibration. If $B, E$ are symplectically aspherical and $\partial F$ is of positive contact type in $F$ then, for $\mu>0$ small enough and $a<0$ arbitrary, the spectral sequence $E_{r}^{p, q}(a, \mu), r \geq$ 2 is canonically isomorphic to the Leray-Serre spectral sequence ${ }_{\mathrm{LS}} E_{r}^{n+p, k+q}$, $n=\frac{1}{2} \operatorname{dim} B, k=\frac{1}{2} \operatorname{dim} F$. In particular, the local system $\mathcal{F H}_{[a, \mu]}^{*}(F)$ is canonically isomorphic to the cohomological local system $\mathcal{H}^{k+*}(F, \partial F)$.

Theorem B can be read as a description of the Leray-Serre spectral sequence in Morse homological terms. A related construction is that of Hutchings [18], with the notable difference that he views the fibration as being a family of fibers, while we view it as being an additional structure on the total space. The resulting generalizations and applications to Floer homology are very different.

The above comparison result yields applications to the Weinstein conjecture. Under the hypothesis of Theorem B, we have $F H_{]-\infty, \mu]}^{*}(E) \simeq$ $H^{*}(E, \partial E)$, and there is a canonical truncation morphism

$$
c^{*}: F H^{*}(E) \longrightarrow H^{*}(E, \partial E),
$$

where $F H^{*}(E):=F H_{]-\infty,+\infty[}^{*}(E)$. The functorial properties of the symplectic cohomology groups $F H_{[a, b]}^{*}(E)$, which are summarized in Section 4.3, force dynamical consequences from algebraic assumptions. The resulting principle concerning the Weinstein conjecture [32] is the following.

Main principle. If the morphism $c^{*}$ is not surjective in maximal degree, then any contact type hypersurface bounding a compact domain in $E$ carries a closed characteristic, i.e., the Weinstein conjecture holds in E. 
In the case $B=\{$ pt. $\}$, this was proved by Viterbo [31]. His proof carries over verbatim to our situation once the groups $F H^{*}(E)$ have been defined and their functorial properties have been established. Following [31], we call the above condition on $c^{*}$ the strong algebraic Weinstein conjecture $(S A W C)$ property.

Remark 1.1. The morphism $c^{*}$ is defined only if the manifold is aspherical and has positive contact type boundary. Thus the hypotheses of Theorem $\mathrm{B}$ are the most general ones under which one can apply the main principle stated above.

Theorem C. Let $F \hookrightarrow E \rightarrow B$ be a negative symplectic fibration. Assume $E, B$ are symplectically aspherical and $F$ has positive contact type boundary. The SAWC property is inherited from the fiber by the total space. In particular, if $F$ satisfies the $S A W C$, then the Weinstein conjecture holds in E.

Proof. Under the assumption that the morphism

$$
F H^{*}(F) \longrightarrow H^{*}(F, \partial F)
$$

is not surjective in degree $2 k=\operatorname{dim} F$, we have to prove that the morphism $F H^{*}(E) \longrightarrow H^{*}(E, \partial E)$ is not surjective in degree $2 n+2 k=\operatorname{dim} E$. Because $H^{2 n+2 k}(E, \partial E) \simeq H^{2 n}\left(B ; \mathcal{H}^{2 k}(F, \partial F)\right)$ and the morphism of spectral sequences $E_{*}^{*, *} \longrightarrow{ }_{\mathrm{LS}} E_{*}^{*, *}$ respects the bigrading, it is enough to show that the map

$$
H^{2 n}\left(B ; \mathcal{F} \mathcal{H}^{k}(F)\right) \longrightarrow H^{2 n}\left(B ; \mathcal{H}^{2 k}(F, \partial F)\right)
$$

is not surjective. We apply Poincaré duality on $B$ and we are left to show that the map $H_{0}\left(B ; \mathcal{F} \mathcal{H}^{k}(F)\right) \longrightarrow H_{0}\left(B ; \mathcal{H}^{2 k}(F, \partial F)\right)$ is not surjective. We remark now that parallel transport in $E$ is symplectic and therefore preserves the orientation of the fibers. This implies that the local system $\mathcal{H}^{2 k}(F, \partial F)$ is trivial and therefore $H_{0}\left(B ; \mathcal{H}^{2 k}(F, \partial F) \simeq H^{2 k}(F, \partial F)\right.$. On the other hand, $H_{0}\left(B ; \mathcal{F} \mathcal{H}^{k}(F)\right)$ is isomorphic to a quotient of $F H^{k}(F)$ (more precisely the quotient by the submodule $F^{\prime}$ generated by elements of the form $\Phi_{\alpha}(u)-u$, where $u \in F H^{k}(F), \alpha \in \pi_{1}(B)$ and $\Phi_{\alpha}$ is the monodromy transformation along $\alpha$ - see Section 7.2 for details on homology with values in a local system). Now the hypothesis implies that the map $F H^{k}(F) / F^{\prime} \longrightarrow H^{2 k}(F, \partial F)$, induced by $F H^{k}(F) \longrightarrow H^{2 k}(F, \partial F)$, is not surjective.

The most important class of symplectic manifolds which satisfy the SAWC condition are subcritical Stein domains. In this case, we actually know by work of Cieliebak [4] that Floer (co)homology is zero. We obtain in particular the following vanishing theorem. 
Theorem D. Let $E$ be a negative symplectic fibration with symplectically aspherical base and subcritical Stein fiber. We have

$$
F H^{*}(E)=0
$$

and the Weinstein conjecture holds in E.

In particular, the Weinstein conjecture holds if $E$ is the unit disc bundle of a Hermitian vector bundle with negative curvature over a symplectically aspherical base.

Proof. By [4] we know that, if the fiber $F$ is subcritical Stein, we have $F H^{*}(F)=0$. The local system giving the $E_{2}$-term of the spectral sequence in Theorem $\mathrm{A}$ has trivial fiber and therefore $E_{2}=0, E_{\infty}=0$ and $F H^{*}(E)=0$.

Remark 1.2. The homological analog of the conclusion in Theorem D is that $F H_{*}(E)=0$. If $E \rightarrow B$ is a negative Hermitian disc bundle as in Definition 3.1, we deduce $F H_{*}^{[\mu, \infty[}(E) \simeq H_{*+n}(E, \partial E) \simeq H_{*+n-2}(B)$ for $n=\frac{1}{2} \operatorname{dim} B$ and $\mu>0$ small enough. We have used here the tautological long exact sequence $F H_{*}(E) \rightarrow F H_{*}^{[\mu, \infty[}(E) \rightarrow H_{*+(n+1)-1}(E, \partial E) \rightarrow$ $F H_{*-1}(E)$ from $[\mathbf{3 1}]$. This fits perfectly into the long exact sequence from $[\mathbf{2}]$ which relates $F H_{*}^{[\mu, \infty[}(E)$ and linearized contact homology $H C_{*}(\partial E) \simeq$ $\bigoplus_{k \geq 0} H_{*-2 k}(B)$.

In the case of a trivial fibration, the spectral sequence degenerates at $E_{2}$ by construction, since the Floer complex on $B \times F$ can be identified with the tensor product of a Morse complex on $B$ with a Floer complex on $F$. The local system $\mathcal{F H}^{*}(F)$ is trivial and we obtain the Künneth formula (see also [25] for a related statement).

Theorem E. Let $B$ and $F$ be symplectically aspherical. We have

$$
F H^{*}(B \times F) \simeq H^{*}\left(B ; F H^{*}(F)\right) .
$$

Remark 1.3. We have $F H^{*}(B \times F)=0$ for any subcritical Stein manifold $F$, hence the Weinstein conjecture holds in $B \times F$ by the main principle stated above. This was first proved by Floer, Hofer, and Viterbo [13] for $F=\mathbb{C}^{\ell}, \ell \geq 1$, which implies the statement for any subcritical $F$ using that such an $F$ is deformation equivalent to a product with $\mathbb{C}[\mathbf{5}]$.

The construction of the spectral sequence is geometric. We choose on $B$ a $C^{2}$-small Morse function $f$ and a generic almost complex structure $J_{B}$. We work on $E$ with Hamiltonians $K$ whose 1-periodic orbits are nondegenerate and concentrated in the fibers lying over the critical points of $f$. We consider on $E$ a modification of the standard Floer equation having the form

$$
u_{s}+J u_{t}=Y \circ u
$$


where $J$ is such that the projection $\pi$ is $\left(J, J_{B}\right)$-holomorphic and $\pi_{*} Y=\nabla f$. This ensures that the map $v=\pi \circ u$ will satisfy the equation

$$
v_{s}+J_{B} v_{t}=\nabla f \circ v .
$$

The main point is that we can choose many such pairs $(K, Y)$ such that the vector field

$$
\mathcal{Y}(x)=J \dot{x}-Y \circ x
$$

defined on the space of contractible loops in $E$ is a strong pseudo-gradient for the action functional $A_{K}$, i.e.,

$$
d A_{K} \cdot \mathcal{Y} \geq \alpha\|\mathcal{Y}\|_{L^{2}}^{2}
$$

for some $\alpha>0$. This ensures that Floer cohomology can be computed by studying moduli spaces of solutions of (1.1) (see Section 4.2). Since the solutions of our pseudo-gradient Floer equation (1.1) on the total space $E$ project to solutions of the Floer equation (1.2) on the base $B$, the Floer complex on $E$ can be filtered by the index of the critical points of $f$. The resulting spectral sequence is the one in Theorem $\mathrm{A}$.

The proof of Theorem B uses that Morse homology is equal to cellular homology as defined in [24, Appendix A.4], provided that the unstable manifolds of the pseudo-gradient vector field give rise to a $\mathrm{CW}$-decomposition of the underlying manifold (see Section 7.2.1). This last fact was shown to be true by Laudenbach [20] under the mild assumption that the pseudogradient vector field is equal near its zeroes to the gradient of a quadratic form with respect to the Euclidean metric. We use Laudenbach's result also in order to construct the Floer local system $\mathcal{F H}_{[a, b]}^{*}(F)$.

Remark 1.4. In the case of a monotone basis, our method of construction of the spectral sequence runs into two kinds of difficulties. The first one is technical and concerns the proof of the pseudo-gradient property, which involves a time-independent metric on the base (see Remark 5.7). The second one is conceptual and concerns the expression of the $E_{2}$-term, which has to encode quantum homological contributions from the base.

The paper is structured as follows. We give in Section 2 the definition and first properties of negative symplectic fibrations. Section 3 contains examples: trivial fibrations, fibrations associated to loops of compactly supported Hamiltonian diffeomorphisms of the fiber, negative vector bundles and convex fibrations as defined in [7, §2.10].

We give in Section 4 the construction of symplectic cohomology groups in a fibered setting, and sketch the dual homological construction in Remark 4.28. The main difficulty of the construction is the proof of a priori $C^{0}$-bounds on Floer trajectories. We emphasize the following two distinctive features of our approach. 
1) We allow admissible Hamiltonians to be "asymptotically linear." This is a much larger class than the ones previously considered in Symplectic homology constructions for arbitrary convex manifolds $[\mathbf{6}, \mathbf{3 1}]$, and generalizes the class of asymptotically quadratic Hamiltonians in [11].

2) We consider a generalization of Floer's equation in which the zeroorder term is modified so that the resulting vector field (1.3) is a pseudo-gradient for the action functional.

We need both these degrees of freedom in order to ensure that solutions of the Floer equation on $E$ project on solutions of the Floer equation on $B$.

Sections 5 to 7 deal with the construction of the spectral sequence. We construct in Section 5 pseudo-gradient vector fields of a special form on the loop space of $E$, and we establish in Section 6 transversality within a geometrically meaningful class of almost complex structures. These technical ingredients are put together in Section 7. The proof of Theorems A and B is given in subsection 7.6. Subsection 7.2, in which we explain how local systems of coefficients can be encoded in the Morse complex, may be of independent interest.

Appendix 8 contains a proof of the (purely linear) fact that a symplectic form $\omega$ is determined, in its conformal class, by the set of $\omega$-compatible almost complex structures. This is referred to in Sections 2 and 3.3.

\section{Negative symplectic fibrations}

Definition 2.1. A locally trivial fibration $F \hookrightarrow E \stackrel{\pi}{\longrightarrow} B$ is called a negative symplectic fibration with contact type boundary fibers (or, for short, negative symplectic fibration) if the following conditions are satisfied.

1) The base $B$ is closed and the fiber $F$ has a nonempty boundary.

2) There exists a 2-form $\Omega \in \Omega^{2}(E, \mathbb{R})$ and a vertical vector field $Z$ defined in a neighborhood of $\partial E$ such that:

- (SYMPLECTIC FIBRATION) $\Omega$ is nondegenerate along the fibers and globally closed;

- (CONTACT TYPE BOUNDARY) $Z$ is outward pointing and transverse to $\partial E$, and satisfies $L_{Z} \Omega=\Omega$;

- (MONODROMY) the horizontal distribution $H=\left(\operatorname{ker} \pi_{*}\right)^{\perp_{\Omega}}$ is tangent to $\partial E$.

3) (NEGATIVITY) there is a symplectic form $\beta$ on $B$ and a nonempty open subset $\mathcal{J}^{\prime} \subset \mathcal{J}(B, \beta)$ such that, in a neighborhood of $\partial E$, we have

$$
\Omega\left(v, \widetilde{J}_{B} v\right) \geq 0
$$

for any $v \in H$ and any almost complex structure $J_{B} \in \mathcal{J}^{\prime}$. Here $\widetilde{J}_{B}$ denotes the lift of $J_{B}$ to $H$ and $\mathcal{J}(B, \beta)$ is the set of almost complex structures $J_{B}$ which are compatible with $\beta$ in the sense that $\beta\left(\cdot, J_{B} \cdot\right)$ defines a Riemannian metric. 
Notations and terminology. The fiber $\pi^{-1}(b)$ at a point $b \in B$ will be denoted either by $E_{b}$ or by $F_{b}$. We shall refer to $H=\left(\operatorname{ker} \pi_{*}\right)^{\perp_{\Omega}}$ as the horizontal distribution or the horizontal connection, while $V=\operatorname{ker} \pi_{*}$ will be called the vertical distribution. As $H$ is tangent to $\partial E$, we have a well-defined parallel transport

$$
\tau_{\gamma}: E_{\gamma(0)} \stackrel{\sim}{\longrightarrow} E_{\gamma(1)}
$$

associated to any continuous path $\gamma:[0,1] \longrightarrow B$. The form $\Omega$ will be called the connection form, while the vector field $Z$ will be called the Liouville vector field or the Liouville vector field in the fibers. We shall refer to a negative symplectic fibration as being a tuple $(E, \pi, B, F, \Omega, Z, \beta)$ or, in order to emphasize the role of $\Omega$ and $Z$, we shall simply refer to it as a triple $(E, \Omega, Z)$.

\section{Remarks.}

1. The hypothesis $d \Omega=0$ implies in particular $d \Omega\left(v_{1}, v_{2}, \cdot\right)=0$ for any $v_{1}, v_{2} \in V$. This is equivalent to the fact that $\tau_{\gamma}$ is a symplectic diffeomorphism, where the symplectic form on $E_{b}$ is $\Omega_{b}=\left.\Omega\right|_{E_{b}}[\mathbf{2 2}$, Lemma 6.11].

2. The total space of a negative symplectic fibration is itself a symplectic manifold, with symplectic form

$$
\omega_{\epsilon}=\pi^{*} \beta+\epsilon \Omega, \quad \epsilon>0 \text { small enough. }
$$

The horizontal distribution is symplectic for $\omega_{\epsilon}$.

3. The conditions $L_{Z} \Omega=\Omega$ and $d \Omega=0$ imply that $\Omega$ is exact in a neighborhood of $\partial E$. The primitive is

$$
\Theta=\iota_{Z} \Omega \text {. }
$$

The restriction of $\Theta$ to each fiber is thus a contact form for the boundary of the fiber. Moreover, the assumption that $Z$ is vertical implies

$$
\left.\Theta\right|_{H} \equiv 0 \text {. }
$$

We denote by $R_{\Theta}$ the Reeb vector field on the boundary of the fibers.

4. The boundary $\partial E$ may fail to be of contact type, as we do not suppose that $\pi^{*} \beta$ is exact in one of its neighborhoods. This phenomenon happens for example in trivial fibrations $E=B \times F$. Nevertheless, as we shall see in Section 4.1, a version of weak pseudo-convexity still holds and that will be enough in order to make use of the maximum principle.

5. Assuming conditions (SYMPLECTIC FIBRATION) and (CONTACT TYPE BOUNDARY), the (MONODROMY) condition is equivalent to the fact that the characteristic distribution for $\omega_{\epsilon}$ on $\partial E$ is tangent to the fibers, and coincides with the characteristic distribution of the restriction of $\Omega$ to the fibers. 
More precisely, let $\ell: H \rightarrow \mathbb{R}$ be the unique linear map such that $Y^{H}+\ell\left(Y^{H}\right) Z \in T(\partial E)$ for every $Y^{H} \in H$. Let $R=R^{V}+R^{H}+$ $\ell\left(R^{H}\right) Z$ be a local generator of the characteristic distribution of $\omega_{\epsilon}$ on $\partial E$, with $R^{V} \in V \cap T(\partial E)$ and $R^{H} \in H$. Then $\ell\left(R^{H}\right)=0$ since $0=\omega_{\epsilon}\left(R, R_{\Theta}\right)=\epsilon \Omega\left(\ell\left(R^{H}\right) Z, R_{\Theta}\right)$ and $\Omega\left(Z, R_{\Theta}\right) \neq 0$. Hence, $0=\omega_{\epsilon}\left(R, Y^{V}\right)=\epsilon \Omega\left(R^{V}, Y^{V}\right)$ for all $Y^{V} \in V \cap T(\partial E)$, so that $R^{V}$ is collinear to $R_{\Theta}$. On the other hand, for any $Y^{H} \in H$, we have $0=$ $\omega_{\epsilon}\left(R, Y^{H}+\ell\left(Y^{H}\right) Z\right)=\omega_{\epsilon}\left(R^{H}, Y^{H}\right)+\epsilon \Omega\left(R^{V}, \ell\left(Y^{H}\right) Z\right)$. Now $R^{V} \neq 0$ because $R \neq 0$ and $H$ is symplectic for $\omega_{\epsilon}$, hence $\Omega\left(R^{V}, Z\right) \neq 0$, which implies that $\ell \equiv 0$ if and only if $R^{H}=0$.

6. The characteristic distribution of the restriction of $\Omega$ to the fibers is preserved by parallel transport. The Reeb dynamics on the boundary of the fibrations that we consider in this paper is therefore of MorseBott type, with the meaning that one closed characteristic on $\partial E_{z}$, $z \in B$ gives rise locally to a family of closed characteristics on $\partial E$ parametrized by an open subset of the base. The reader has to keep in mind this geometric picture as a motivation for the construction of the geometric Hamiltonians in Section 5.2.

We denote by $\operatorname{Spec}(\partial E)$ the set of periods of closed characteristics on $\partial E$ normalized by the 1 -form $\Theta=\iota_{Z} \Omega$.

7. Let $\varphi_{t}$ be the flow of $Z$. We can trivialize a neighborhood $\mathcal{U}$ of $\partial E$ by the diffeomorphism

$$
\begin{gathered}
\Psi: \partial E \times[1-\delta, 1] \longrightarrow \mathcal{U}, \\
(p, S) \longmapsto \varphi \ln S(p) .
\end{gathered}
$$

The condition $L_{Z} \Omega=\Omega$ translates into $\varphi_{t}^{*} \Omega=e^{t} \Omega$. If $\Theta \mid$ denotes the restriction of $\Theta$ to $\partial E$, we have $\Psi^{*} \Theta=S \Theta \mid$ and

$$
\Psi^{*} \Omega=d(S \Theta \mid) .
$$

We can therefore complete $E$ to a fibration

$$
\widehat{E}=E \bigcup_{\Psi} \partial E \times[1, \infty[
$$

and define the connection form $\widehat{\Omega}$ on $\widehat{E}$ by

$$
\widehat{\Omega}= \begin{cases}\Omega & \text { on } E, \\ d(S \Theta \mid) & \text { on } \partial E \times[1, \infty[.\end{cases}
$$

The Liouville vector field $Z$ is transformed by $\Psi$ into $S \frac{\partial}{\partial S}$ on $\partial E \times$ $[1-\delta, 1]$. We extend it to $\partial E \times\left[1, \infty\left[\right.\right.$ as $S \frac{\partial}{\partial S}$ and we denote the extended vector field by $\widehat{Z}$.

The (MONODROMY) condition implies that the horizontal distribution on $\widehat{E}$ is tangent to every level set $S=$ ct, $S \geq 1-\delta$. This follows 
from the fact that $\varphi_{t}$ preserves $H$, hence the latter is invariant under the flow of $S \frac{\partial}{\partial S}$ (or, equivalently, of $\frac{\partial}{\partial S}$ ). We therefore have

$$
\left.d S\right|_{H} \equiv 0 .
$$

8. One must note that the construction of the manifold $\widehat{E}$ only makes use of the (SYMPLECTIC FIBRATION) and (CONTACT TYPE BOUNDARY) conditions, while the (NEGATIVITY) condition ensures that the 2 -form $\widehat{\omega}_{\epsilon}=\pi^{*} \beta+\epsilon \widehat{\Omega}$ on $\widehat{E}$ is symplectic for $\epsilon>0$ small enough.

Conversely, let us start with a fibration $\widehat{E}$ endowed with a 2-form $\widehat{\Omega}$ and a vertical vector field $\widehat{Z}$ which is complete at infinity, satisfying the (SYMPLECTIC FIBRATION), (CONTACT TYPE BOUNDARY) and (NEGATIVITY) conditions. A compact hypersurface $\Sigma \subset \widehat{E}$ that trivializes through the flow of $\widehat{Z}$ a neighborhood of infinity as $\Sigma \times[1, \infty[$ will be called a trivializing hypersurface. The choice of any such $\Sigma$ gives rise to a fibration $E=\overline{\operatorname{int} \Sigma}$ which satisfies the same three conditions above.

9. We have imposed the (MONODROMY) condition on $E$ in order for the monodromy to be well defined as a symplectic diffeomorphism of the fiber. Note however that, if one starts directly with $\widehat{E}$ as above, the natural condition under which monodromy is well defined is some uniform nonverticality assumption on $H$, strictly weaker than the requirement $\left.d S\right|_{H} \equiv 0$. Our choice is motivated by the fact that trivializing hypersurfaces $\Sigma$ such that $\left.d S\right|_{\Sigma} \equiv 0$ are a crucial ingredient in the proof of a priori $C^{0}$-bounds for the admissible Hamiltonians on $\widehat{E}$ that we define in Section 4.1.

The following result gives a geometric criterion for the (MONODROMY) condition.

Proposition 2.2. Let $(\widehat{E}, \widehat{\Omega}, \widehat{Z})$ be a fibration satisfying conditions (SYMPLECTIC FIBRATION) and (CONTACT TYPE BOUNDARY), with $\widehat{Z}$ complete at infinity. There is a choice of a trivializing hypersurface $\Sigma$ in $\widehat{E}$ such that $H$ is tangent to $\Sigma$ if and only if the monodromy of $\widehat{E}$ admits an invariant trivializing hypersurface in the fiber.

Proof. Assume first that $H$ is tangent to $\Sigma$. For any loop $\gamma$ on $B$ based at $b$, the monodromy will send $\Sigma_{b}=\Sigma \cap E_{b}$ diffeomorphically to itself and $\Sigma_{b}$ is obviously a trivializing hypersurface in $E_{b}$.

Conversely, let $\Sigma_{b} \subset E_{b}$ be an invariant trivializing hypersurface. Define $\Sigma=\bigcup_{b^{\prime} \in B} \tau_{\gamma_{b b^{\prime}}}\left(\Sigma_{b}\right)$, where $\gamma_{b b^{\prime}}$ is an arbitrary path from $b$ to $b^{\prime}$. It is enough to prove that $\tau_{\gamma_{b b^{\prime}}}\left(\Sigma_{b}\right)$ is independent of $\gamma_{b b^{\prime}}$ in order to infer that $\Sigma$ is smooth and $H \subset T \Sigma$. Let therefore $\widetilde{\gamma}_{b b^{\prime}}$ be another path running from $b$ to $b^{\prime}$. We have

$$
\tau_{\widetilde{\gamma}_{b b^{\prime}}}\left(\Sigma_{b}\right)=\tau_{\gamma_{b b^{\prime}}} \circ \tau_{\gamma_{b b^{\prime}}^{-1} \cdot \widetilde{\gamma}_{b b^{\prime}}}\left(\Sigma_{b}\right)=\tau_{\gamma_{b b^{\prime}}}\left(\Sigma_{b}\right) .
$$


The last equality makes use of the fact that $\Sigma_{b}$ is monodromy invariant.

10. One may strengthen the (NEGATIVITY) condition by requiring $\Omega$ to be nonnegative on $H$ for all $\widetilde{J}_{B}$ where $J_{B} \in \mathcal{J}(B, \beta)$. Appendix 8 shows that this is a very strong assumption: either $\Omega$ is non-degenerate on $H$ and then it is proportional to $\pi^{*} \beta$, or $H$ splits at each point into a direct sum of two subspaces which are symplectic for $\pi^{*} \beta$ and such that $\Omega$ vanishes on one of them and is proportional to $\pi^{*} \beta$ on the other.

Nevertheless, requiring $\Omega$ to tame only the almost complex structures belonging to some nonempty open subset of $\mathcal{J}(B, \beta)$ is enough for the subsequent transversality issues. If the restriction of $\Omega$ to $H$ is non-degenerate on $\partial E$, this follows by imposing the (NEGATIVITY) condition to be true only for one almost complex structure $J_{B}$.

\section{Examples}

3.1. Products. Trivial fibrations $E=B \times F$ with $B$ closed symplectic and $F$ symplectic with contact type boundary are negative symplectic fibrations in the sense of Definition 2.1. Let $\pi_{B}, \pi_{F}$ denote the projections on the two factors, let $\Omega_{F}$ be the symplectic form on $F$ and $Z_{F}$ be the Liouville vector field on $F$, defined in a neighborhood of $\partial F$. Then $\Omega=\pi_{F}^{*} \Omega_{F}$ and $Z=\left(0, Z_{F}\right) \in T B \times T F \simeq T(B \times F)$ make $E$ into a negative symplectic fibration. We have $H=T B \times\{0\} \subset T(B \times F)$ and $\left.\Omega\right|_{H} \equiv 0$, so the negativity condition is trivially satisfied.

3.2. Hamiltonian diffeomorphisms. Let $F \hookrightarrow E \stackrel{\pi}{\longrightarrow} B$ be a Hamiltonian fibration with contact type boundary fibers and structure group $\operatorname{Ham}(F, \partial F)$, the group of Hamiltonian diffeomorphisms which fix a neighborhood of $\partial F$. A Hamiltonian fibration admits a canonical coupling form $\Omega$, which in our situation vanishes on the horizontal distribution near the boundary (see [16]) and makes $E$ into a negative symplectic fibration. As a special case, we mention fibrations $F \hookrightarrow E \longrightarrow \mathbb{S}^{2}$ defined by elements of $\pi_{1}(\operatorname{Ham}(F, \partial F))$.

\subsection{Negative line bundles.}

Definition 3.1. A complex line bundle $\mathcal{L} \longrightarrow B$ over a closed symplectic manifold $(B, \beta)$ is called negative if it admits a Hermitian metric $h$ and a Hermitian connection $\nabla$ such that the curvature $\frac{1}{2 i \pi} F^{\nabla} \in \Omega^{2}(B, \mathbb{R})$ is negative:

$$
\frac{1}{2 i \pi} F^{\nabla}\left(v, J_{B} v\right)<0
$$

for any $J_{B} \in \mathcal{J}(B, \beta)$ and any nonzero vector $v \in T B$. 
Remark 3.2. The 2 -form $-\frac{1}{2 i \pi} F^{\nabla}$ is a symplectic form on $B$ representing $-c_{1}(\mathcal{L})$. Moreover, it tames all almost complex structures that are tamed by $\beta$ and this ensures that $-\frac{1}{2 i \pi} F^{\nabla}$ and $\beta$ are proportional by a positive constant if $\operatorname{dim} B \geq 4$, or by a positive function if $\operatorname{dim} B=2$ (cf. Appen$\operatorname{dix} 8$ ). In particular, we have $c_{1}(\mathcal{L})=-\lambda[\beta], \lambda>0$ (if $\operatorname{dim} B=2$, this is true because $\left.\operatorname{dim} H^{2}(B, \mathbb{R})=1\right)$. Conversely, assume $c_{1}(\mathcal{L})=-\lambda[\beta]$, $\lambda>0$. Then $-\lambda \beta$ represents $c_{1}(\mathcal{L})$ and, for any Hermitian metric $h$ on $\mathcal{L}$, one can find a Hermitian connection $\nabla$ such that $\frac{1}{2 i \pi} F^{\nabla}=-\lambda \beta$. In particular, $-\frac{1}{2 i \pi} F^{\nabla}$ tames the same almost complex structures as $\beta$. We have just proved that Definition 3.1 is equivalent to

Definition 3.3. A complex line bundle $\mathcal{L} \longrightarrow B$ over a closed symplectic manifold $(B, \beta)$ is negative if there exists $\lambda>0$ such that

$$
c_{1}(\mathcal{L})=-\lambda[\beta] \text {. }
$$

We note here that the topological type of $\mathcal{L}$ is uniquely determined by the choice of an integral lift of $-\lambda[\beta]$. The preceding discussion shows in particular that, up to a change of connection, we can assume that $-\frac{1}{2 i \pi} F^{\nabla}=$ $\lambda \beta, \lambda>0$.

Any linear connection $\nabla$ determines a transgression 1 -form $\theta^{\nabla} \in \Omega^{1}(\mathcal{L} \backslash$ $\left.0_{\mathcal{L}}, \mathbb{R}\right)$. Its definition is the following $[\mathbf{1 4}]$ :

$$
\left\{\begin{array}{l}
\theta_{u}^{\nabla}(u)=0, \quad \theta_{u}^{\nabla}(i u)=1 / 2 \pi, \quad u \in \mathcal{L} \backslash 0_{\mathcal{L}} ; \\
\left.\theta^{\nabla}\right|_{H^{\nabla}} \equiv 0, \text { with } H^{\nabla} \text { the horizontal distribution defining } \nabla .
\end{array}\right.
$$

The transgression form is a primitive for $-\pi^{*}\left(\frac{1}{2 i \pi} F^{\nabla}\right)$. In our case, this means

$$
d \theta^{\nabla}=\lambda \pi^{*} \beta .
$$

On the other hand, the restriction of $\theta^{\nabla}$ to the fibers equals, up to the factor $\frac{1}{2 \pi}$, the angular form. If $r(u)=|u|$ is the radial coordinate in the fibers, we infer that

$$
\Omega=d\left(r^{2} \theta^{\nabla}\right)
$$

equals $\frac{1}{\pi} d$ Area along the fibers. Moreover, $\Omega$ extends to a smooth form on $\mathcal{L}$ by $\Omega_{z}(\xi, \cdot)=0, \xi \in T_{z} 0_{\mathcal{L}}$ and $\left.\Omega_{z}\right|_{\mathcal{L}_{z}}=\frac{1}{\pi} d$ Area, $z \in 0_{\mathcal{L}}$. This follows from the expansion $\Omega=d r^{2} \wedge \theta^{\nabla}+r^{2} \lambda \pi^{*} \beta$, with $d r^{2} \wedge \theta_{u}^{\nabla}(\xi, \cdot)=0, \xi \in H_{u}^{\nabla}$ and $\left.d r^{2} \wedge \theta_{u}^{\nabla}\right|_{\mathcal{L}_{\pi(u)}}=\frac{1}{\pi} d$ Area, $u \in \mathcal{L} \backslash 0_{\mathcal{L}}$. The vertical vector field

$$
Z(u)=\frac{u}{2}
$$

satisfies $\iota_{Z} \Omega=r^{2} \theta^{\nabla}$, hence $L_{Z} \Omega=\Omega$. We define

$$
E=\{u \in \mathcal{L}:|u| \leq 1\} .
$$

We claim that $E$ together with $\Omega$ and $Z$ as above is a negative symplectic fibration in the sense of Definition 2.1. The (SYMPLECTIC FIBRATION) and 
(CONTACT TYPE BOUNDARY) conditions are clear by construction. The connection being Hermitian, parallel transport preserves the length of vectors in $\mathcal{L}$, hence the (MONODROMY) condition is also satisfied. The (NEGATIVITY) condition follows from the expansion of $\Omega$, which implies $\left.\Omega\right|_{H^{\nabla}}=r^{2} \lambda \pi^{*} \beta$ with $r^{2} \lambda \geq 0$.

Remark 3.4. The dual $\mathcal{L}^{*}$ of an ample line bundle $\mathcal{L}$ over a complex manifold $B$ is a negative line bundle in the sense of Definition 3.3, with the meaning that there is a symplectic form $\beta$ on $B$ such that $c_{1}\left(\mathcal{L}^{*}\right)=-\lambda[\beta], \lambda>0$. Indeed, Kodaira's embedding theorem ensures the existence of an embed$\operatorname{ding} \phi_{m}: B \hookrightarrow \mathbb{P}^{N}$ given by the sections of $\mathcal{L}^{\otimes m}$ such that $\phi_{m}^{*} \mathcal{O}(1)=\mathcal{L}^{\otimes m}$. Let $\omega_{\mathrm{FS}}$ be the Fubini-Study form on $\mathbb{P}^{N}$ representing $c_{1}(\mathcal{O}(1))$, and define $\beta=\phi_{m}^{*} \omega_{\mathrm{FS}}$. Then

$$
c_{1}\left(\mathcal{L}^{*}\right)=-\frac{1}{m} c_{1}\left(\mathcal{L}^{\otimes m}\right)=-\frac{1}{m} \phi_{m}^{*} c_{1}(\mathcal{O}(1))=-\frac{1}{m}\left[\phi_{m}^{*} \omega_{\mathrm{FS}}\right]=-\frac{1}{m}[\beta] .
$$

3.4. Negative vector bundles. Our discussion in this section follows Griffiths [15] and Kobayashi [19].

Definition 3.5. A complex vector bundle $E \stackrel{\pi}{\longrightarrow} B$ over a closed symplectic manifold $(B, \beta)$ is called negative if it admits a Hermitian metric $h$ and a Hermitian connection $\nabla$ such that the curvature $\frac{1}{i} F^{\nabla} \in \Omega^{2}(B$, End $E)$ is negative definite as a Hermitian matrix:

$$
\frac{1}{i} F^{\nabla}\left(v, J_{B} v\right)<0
$$

for any $J_{B} \in \mathcal{J}(B, \beta)$ and any nonzero vector $v \in T B$.

Remarks 3.6. Negative projectively flat bundles (with curvature $-\beta \mathrm{Id}$ ) are a particular case of negative vector bundles.

The projectivized bundle associated to $E$ is

$$
\mathbb{P}(E)=\left\{(b,[v]): b \in B, v \in E_{b} \backslash\{0\}\right\} .
$$

Let $p: \mathbb{P}(E) \longrightarrow B$ and $\bar{p}: p^{*} E \longrightarrow E$ be the induced projection and the corresponding bundle map. The tautological bundle $\mathcal{L}_{E} \longrightarrow \mathbb{P}(E)$ is the subbundle of $p^{*} E$ defined as

$$
\mathcal{L}_{E}=\{(b,[v], \lambda v):(b,[v]) \in \mathbb{P}(E), \lambda \in \mathbb{C}\} .
$$

Let $i: \mathcal{L}_{E} \longrightarrow p^{*} E$ be the canonical inclusion and $\Phi=\bar{p} \circ i$. Then

$$
\Phi: \mathcal{L}_{E} \backslash 0_{\mathcal{L}_{E}} \stackrel{\sim}{\longrightarrow} E \backslash 0_{E}
$$

is a diffeomorphism (respectively a biholomorphism if the bundle $E \longrightarrow B$ is holomorphic). Its inverse is (see the diagram below)

$$
\begin{gathered}
\Psi: E \backslash 0_{E} \stackrel{\sim}{\longrightarrow} \mathcal{L}_{E} \backslash 0_{\mathcal{L}_{E}}, \\
(b, v) \longmapsto(b,[v], v) .
\end{gathered}
$$


We show now that, under the negativity condition on $E$, the line bundle $\mathcal{L}_{E}$ is negative in the sense that there is a canonical connection $\nabla$ which preserves the induced Hermitian metric on $\mathcal{L}_{E}$, as well as a canonical symplectic form on $\mathbb{P}(E)$, such that Definition 3.1 is satisfied. Moreover, if $\Omega$ and $Z$ are constructed on $\mathcal{L}_{E}$ as in the previous section, their pull-backs $\Omega_{E}=\Psi^{*} \Omega$ and $Z_{E}=\Psi^{*} Z$, defined a priori only on $E \backslash 0_{E}$, extend smoothly to $E$ and make

$$
D E=\{v \in E:|v| \leq 1\}
$$

into a negative symplectic fibration.

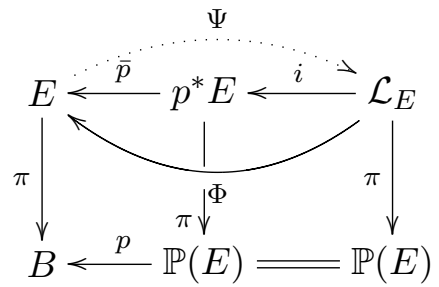

3.4.1. Connection on $\mathcal{L}_{E}$. We write $\mathbb{P}(E)_{b}$ for the fiber $p^{-1}(b), b \in B$ and, for a connection $D$, we denote by $H^{D}$ the associated horizontal distribution, or simply $H$ if there is no danger of confusion.

The connection $\nabla$ canonically defines a parallel transport in $\mathbb{P}(E)$ and hence a horizontal distribution $H_{\mathbb{P}(E)}$ with monodromy in $P G L(r), r=$ $\operatorname{rk}(E)$. The horizontal distribution $H$ associated to any connection on $p^{*} E$ canonically decomposes as

$$
H=H_{\text {fiber }} \oplus H_{\text {base }},
$$

where the subspaces $H_{\text {fiber }}$ and $H_{\text {base }}$ of $H$ are uniquely determined by the conditions

$$
p_{*} \circ \pi_{*} H_{\text {fiber }}=0, \quad \pi_{*} H_{\text {base }}=H_{\mathbb{P}(E)} .
$$

Note that $H_{\text {fiber }} \subset T\left(\left.p^{*} E\right|_{\mathbb{P}(E)_{b}}\right)$ and $H_{\text {base }} \pitchfork T\left(\left.p^{*} E\right|_{\mathbb{P}(E)_{b}}\right), b \in B$. We call them respectively the components of $H$ along the fibers of $\mathbb{P}(E)$ and along the base $B$. The distribution $H_{\text {fiber }}$ defines a linear connection on every $\left.p^{*} E\right|_{\mathbb{P}(E)_{b}}, b \in B$.

We can give further details on the preceding decomposition for the induced connection $p^{*} \nabla$. The associated horizontal distribution is $H^{p^{*} \nabla}=$ $\left(\bar{p}_{*}\right)^{-1} H^{\nabla}$. Any choice of frame $\left(e_{1}, \ldots, e_{r}\right)$ in $E_{b}$ gives rise to a trivialization

$$
\begin{gathered}
\mathbb{P}(E)_{b} \times\left.\mathbb{C}^{r} \stackrel{\sim}{\longrightarrow} p^{*} E\right|_{\mathbb{P}(E)_{b}}, \\
\left(b,[v],\left(\lambda_{1}, \ldots, \lambda_{r}\right)\right) \longmapsto\left(b,[v], \lambda_{1} e_{1}+\cdots+\lambda_{r} e_{r}\right) .
\end{gathered}
$$

The associated flat connection does not depend on the choice of frame and its horizontal distribution is precisely $H_{\text {fiber }}^{p^{*}}$.

Let us now go to $\mathcal{L}_{E}$. Its restriction to any $\mathbb{P}(E)_{b}$ is clearly not preserved by parallel transport along $H_{\text {fiber }}^{p^{*} \nabla}$, otherwise $\left.\mathcal{L}_{E}\right|_{\mathbb{P}(E)_{b}}$ would be trivial. 
On the other hand, $\mathcal{L}_{E}$ is preserved, together with the induced Hermitian metric, by parallel transport along $H_{\text {base }}^{p^{*} \nabla}$. This follows from the fact that $\pi_{*} H_{\text {base }}=H_{\mathbb{P}(E)}$.

We want to associate to $h$ and $\nabla$ in a canonical way a connection $\widetilde{\nabla}$ on $\mathcal{L}_{E}$ which preserves the induced Hermitian metric. Its horizontal distribution decomposes as $\widetilde{H}=\widetilde{H}_{\text {fiber }} \oplus \widetilde{H}_{\text {base }}$ and we define

$$
\widetilde{H}_{\text {base }}=H_{\text {base }}^{p^{*} \nabla} \text {. }
$$

Let us define $\widetilde{H}_{\text {fiber }}$. Each restriction $\left.\mathcal{L}_{E}\right|_{\mathbb{P}(E)_{b}}, b \in B$ is isomorphic (as a Hermitian bundle) to the canonical bundle $\mathcal{O}(-1) \longrightarrow \mathbb{P}^{r-1}$ endowed with the canonical Hermitian metric. The isomorphism is given by the choice of some frame in $E_{b}$ which is orthonormal with respect to $h$. The Chern connection on $\mathcal{O}(-1)$ is invariant under the action of $P S U(r)$, and this implies that the induced connection on $\mathcal{L}_{E} \mid \mathbb{P}(E)_{b}$ is independent of the choice of orthonormal frame. We define its horizontal distribution to be $\widetilde{H}_{\text {fiber. }}$. With a slight abuse of notation, we can write the decomposition $\widetilde{H}=\widetilde{H}_{\text {fiber }} \oplus \widetilde{H}_{\text {base }}$ as

$$
\widetilde{H}=H_{\mathcal{O}(-1)} \oplus H_{\text {base }}^{p^{*} \nabla} .
$$

3.4.2. Symplectic form on $\mathbb{P}(E)$. The curvature of the Chern connection on $\mathcal{O}(-1)$ is $-\omega_{\mathrm{FS}}$, with $\omega_{\mathrm{FS}}$ the Fubini-Study form normalized by $\left\langle\left[\omega_{\mathrm{FS}}\right],\left[\mathbb{C} P^{1}\right]\right\rangle=1$. We infer that

$$
\omega=-\frac{1}{2 i \pi} F^{\tilde{\nabla}}
$$

is a 2 -form which restricts to $\omega_{\mathrm{FS}}$ on every fiber $\mathbb{P}(E)_{b}, b \in B$. We claim that $\omega$ is actually nondegenerate on $\mathbb{P}(E)$. This will define our preferred symplectic form on $\mathbb{P}(E)$.

First, we show that $T\left(\mathbb{P}(E)_{b}\right)$ and $H_{\mathbb{P}(E)}$ are orthogonal with respect to $\omega$. This amounts to proving that $H_{\mathcal{O}(-1)}$ and $H_{\text {base }}^{p^{*} \nabla}$ are in involution, as the value of the curvature at two vectors is given by the vertical projection of the Lie bracket of their horizontal lifts (see e.g., [14]).

Let $u(s, t), s, t \in[0,1]$ be a parametrized surface on $\mathbb{P}(E)$ such that

- $u(\cdot, 0)$ is tangent to some $\mathbb{P}(E)_{b}, b \in B$;

- $u(\cdot, t)$ is the parallel transport of $u(\cdot, 0)$ along some curve $\gamma$ on $B$ with $\gamma(0)=b$.

Let us fix a point $q \in \mathcal{L}_{E, u(0,0)}$ and a horizontal lift $\widetilde{u}$ of $u(\cdot, 0)$ at $q$. This allows to lift horizontally every curve $u(s, \cdot)$ with initial point $\widetilde{u}(s, 0)$. We still denote by $\widetilde{u}$ the resulting lift of $u(\cdot, \cdot)$ and we have to show that every $\widetilde{u}(\cdot, t)$ is horizontal.

This amounts to show that $H_{\mathcal{O}(-1)}$ is preserved by parallel transport along $H_{\text {base }}^{p^{*} \nabla}$. But $H_{\mathcal{O}(-1)}$ corresponds via the isomorphism $\mathbb{C}^{n} \backslash\{0\} \simeq \mathcal{O}(-1) \backslash$ 
$0_{\mathcal{O}(-1)}$ to the distribution of hyperplanes $(\mathbb{C} \cdot v)^{\perp}, v \in \mathbb{C}^{n} \backslash\{0\}$, which is clearly preserved by Hermitian parallel transport in $E$. The latter in turn corresponds to parallel transport along $H_{\text {base }}^{p^{*} \nabla}$ in $\mathcal{L}_{E}$.

Secondly, we show that the negativity condition in Definition 3.5 is equivalent to the fact that $\omega$ is positive on $H_{\mathbb{P}(E)}$, with the meaning that $\omega\left(X, \widetilde{J}_{B} X\right)>0$ for any nonzero vector $X \in H_{\mathbb{P}(E)}$, where $\widetilde{J}_{B}$ is the lift to $H_{\mathbb{P}(E)}$ of an almost complex structure $J_{B}$ compatible with $\beta$. We denote $X^{\prime}=p_{*} X$ and we have

$$
\begin{aligned}
-\frac{1}{i} F_{(b,[v])}^{\widetilde{\widetilde{V}}}\left(X, \widetilde{J}_{B} X\right) & =-\frac{1}{i|v|^{2}} t \bar{v} \cdot F^{p^{*} \nabla}\left(X, \widetilde{J}_{B} X\right) \cdot v \\
& =-\frac{1}{i|v|^{2}} \bar{v} \cdot F^{\nabla}\left(X^{\prime}, J_{B} X^{\prime}\right) \cdot v>0 .
\end{aligned}
$$

This shows that $\mathcal{L}_{E}$ is a negative line bundle in the sense of Definition 3.1. If $\theta^{\widetilde{\nabla}}$ is the transgression 1-form associated to $\widetilde{\nabla}$, then the connection 2-form and the Liouville vector field on $\mathcal{L}_{E}$ are

$$
\Omega=d\left(r^{2} \theta^{\tilde{\nabla}}\right), \quad Z(u)=\frac{u}{2} .
$$

3.4.3. Connection form and Liouville vector field on $\boldsymbol{E}$. We define

$$
\Omega_{E}=\Psi^{*} \Omega, \quad Z_{E}=\Psi^{*} Z .
$$

We claim that $\Omega_{E}$ and $Z_{E}$ extend smoothly to the whole of $E$ and they verify Definition 3.5. The key step is to consider

$$
\Theta=\Psi^{*}\left(r^{2} \theta^{\widetilde{\nabla}}\right) \text {. }
$$

We clearly have $\left.\Theta\right|_{E_{b} \backslash\{0\}}=\Psi^{*}\left(\left.r^{2} \theta^{\widetilde{\nabla}}\right|_{\mathbb{P}(E)_{b}}\right)$ and we claim that this is the positive $U(r)$-invariant Liouville form on $E_{b}$. By choosing a unitary frame on $E_{b}$, we can work within the explicit model of the biholomorphism $\mathbb{C}^{r} \backslash$ $\{0\} \simeq \mathcal{O}(-1) \backslash 0_{\mathcal{O}(-1)}, v \longmapsto([v], v)$. We have already mentioned that the horizontal distribution of the Chern connection on $\mathcal{O}(-1)$ corresponds to the distribution of hyperplanes $(\mathbb{C} \cdot v)^{\perp}, v \in \mathbb{C}^{r} \backslash\{0\}$, and we therefore have $\left.\Theta_{v}\right|_{(\mathbb{C} \cdot v)^{\perp}} \equiv 0, \Theta_{v}(v)=0, \Theta_{v}(i v)=\frac{|v|^{2}}{2 \pi}$, or else stated

$$
\Theta_{v}=\frac{1}{2 \pi}\langle i v, \cdot\rangle=\frac{1}{2 \pi} \sum_{j=1}^{r} x_{j} d y_{j}-y_{j} d x_{j} .
$$

As a consequence, $\Theta$ extends smoothly over the origin in every fiber. But it is clear that this argument can be performed in families and the extension is smooth on $E$ so that $d \Theta$ is a smooth extension of $\Omega_{E}$ which is closed and equal to $\frac{1}{\pi} d$ Area in the fibers. It is also clear that $Z_{E}$ extends smoothly by 0 over $0_{E}$, with $\iota_{Z_{E}} \Omega_{E}=\Theta$. This accounts for the (SYMPLECTIC FIBRATION) and (CONTACT TYPE BOUNDARY) conditions. The (MONODROMY) condition is automatic as the connection $\nabla$ was supposed from the very beginning 
to be Hermitian. In order to verify the (NEGATIVITY) condition, let us recall that $\Phi_{*} \widetilde{H}_{\text {base }}=H^{\nabla}$. We then have

$$
\left.\left(\Psi^{*} \Omega\right)\right|_{H^{\nabla}}=\Psi^{*}\left(\left.\Omega\right|_{\widetilde{H}_{\text {base }}}\right)=\Psi^{*}\left(\left.\lambda r^{2}\left(\pi^{*} \omega\right)\right|_{\widetilde{H}_{\text {base }}}\right)=\lambda r^{2} \Psi^{*} \pi^{*}\left(\left.\omega\right|_{H_{\mathbb{P}(E)}}\right) .
$$

Let $J_{B} \in \mathcal{J}(B, \beta)$ and denote $\widetilde{J}_{B}$ the lift to $H^{\nabla}$ and $J_{B}^{\prime}$ the lift to $H_{\mathbb{P}(E)}$. Let $X$ be a vector field on $B$ and denote $\widetilde{X}$ the lift to $H^{\nabla}$ and $X^{\prime}$ the lift to $H_{\mathbb{P}(E)}$. We have $\pi_{*} \Psi_{*} \widetilde{X}=X^{\prime}$ and therefore

$$
\Omega_{E}\left(\widetilde{X}, \widetilde{J}_{B} \widetilde{X}\right)=\Psi^{*} \Omega\left(\widetilde{X}, \widetilde{J}_{B} \widetilde{X}\right)=\lambda r^{2} \omega\left(X^{\prime}, J_{B}^{\prime} X^{\prime}\right),
$$

or

$$
\left.\Omega_{E}\right|_{(b, v)}\left(\widetilde{X}, \widetilde{J}_{B} \widetilde{X}\right)=-\frac{1}{2 i \pi}{ }^{t} \bar{v} \cdot F^{\nabla}\left(X, J_{B} X\right) \cdot v .
$$

The last expression is positive for $v \neq 0$.

Remark 3.7. The case of projectively flat negative vector bundles (with curvature equal to $-i \beta \mathrm{Id}$ ) corresponds precisely to a connection form $\Omega_{E}$ which depends only on $|v|$ (and, of course, on $b$ ).

3.5. Convex fibrations. We explain now a variation on an example from $[\mathbf{7}, \S 2.10]$. Let $G$ be a compact Lie group with Lie algebra $\mathfrak{g}$. Let $X \stackrel{\pi}{\longrightarrow} B$ be a principal $G$-bundle with connection $\theta_{A} \in \Omega^{1}(X, \mathfrak{g})$. We denote by $F_{A} \in \Omega^{2}\left(B, X \times_{\text {ad }} \mathfrak{g}\right)$ its curvature and by Hor $_{A}$ its horizontal distribution. We assume that $B$ is symplectic with symplectic form $\beta$.

Let $\left(F, \omega_{F}\right)$ be a symplectic manifold endowed with a Hamiltonian action of $G$ with moment map $\phi_{F}: F \longrightarrow \mathfrak{g}^{*}$. We impose the following conditions.

- (G-CONTACT TYPE BOUNDARY) The boundary $\partial F$ is $G$-invariant and admits a $G$-invariant Liouville vector field $Z$ which is also conformal for the moment map:

$$
d \phi_{F} \cdot Z=\phi_{F} .
$$

- (G-NEGATIVITY) There exists $J_{B} \in \mathcal{J}(B, \beta)$ such that

$$
\left\langle F_{A}\left(X, J_{B} X\right), \phi_{F}(f)\right\rangle \leq 0
$$

for all $X \in \operatorname{Hor}_{A}$ and $f$ in a neighborhood of $\partial F$.

Remark 3.8. The ( $G$-NEGATIVITY) condition is related to Weinstein's notion of fat bundles, i.e., $G$-principal bundles admitting a connection $\theta_{A}$ such that the 2-form $\left\langle F_{A}(\cdot, \cdot), \eta\right\rangle$ is nondegenerate for all nonzero $\eta \in \mathfrak{g}^{*}$. The ( $G$-NEGATIVITY) condition also plays a crucial role in $[\mathbf{7}]$.

We claim that the associated bundle

$$
X_{F}=X \times_{G} F
$$


is a negative symplectic fibration. In order to see this, we recall Weinstein's construction of symplectic fibrations through symplectic reduction as explained in $[\mathbf{1 6}, \S 2]$. The connection $\mathrm{Hor}_{A}$ defines the subbundle

$$
M=\left\{\eta \in T^{*} X:\left.\eta\right|_{\operatorname{Hor}_{A}}=0\right\} \subset T^{*} X .
$$

One can show that $M \simeq X \times \mathfrak{g}^{*}$. In any case, $M$ inherits from $T^{*} X$ a 2 -form $\omega_{\Gamma}$ which restricts to the canonical symplectic form on the fibers $X_{b} \times \mathfrak{g}^{*} \simeq T^{*} G$. Then $M \times F$ is a Hamiltonian $G$-space with moment map

$$
\phi(x, \eta, f)=\phi_{F}(f)+\eta \text {. }
$$

The zero set $\phi^{-1}(0)$ is naturally identified with $X \times F$ and the symplectic reduction $\phi^{-1}(0) / G$ is isomorphic to $X_{F}$. The (pre)symplectic form $\omega_{\Gamma}+\omega_{F}$ on $M \times F$ is $G$-invariant, and the same is true for its restriction to $\phi^{-1}(0)$. Moreover, for any $\xi \in \mathfrak{g}$, we have $\iota\left(\xi_{M}, \xi_{F}\right)\left(\omega_{\Gamma}+\omega_{F}\right)=-d\left\langle\phi_{F}+\mathrm{pr}_{2}, \xi\right\rangle=0$ on $\phi^{-1}(0)$, hence $\left.\left(\omega_{\Gamma}+\omega_{F}\right)\right|_{\phi^{-1}(0)}$ is the pull-back of a (pre)symplectic form on $M \times_{G} F=X_{F}$ which we denote by $\omega_{\Gamma, F}$. We have denoted by $\xi_{M}, \xi_{F}$ the infinitesimal generators of the action of $G$ on $M$ and $F$, respectively. The form $\omega_{\Gamma, F}$ restricts to the symplectic form $\omega_{F}$ in the fibers of $X_{F}$. We define

$$
\Omega=\omega_{\Gamma, F} .
$$

The (NEGATIVITY) condition for $\omega_{\Gamma, F}$ is now equivalent to the (GNEGATIVITY) condition above because $\omega_{\Gamma, F}$ acts at a point $[x, f] \in X_{F}$ as $-\left\langle\phi_{F}(f), F_{A}(x)(\cdot, \cdot)\right\rangle$ (cf. [16]). One can also prove that the horizontal distribution of $\omega_{\Gamma, F}$ is the distribution induced by the connection $\theta_{A}$. On the other hand, parallel transport $\tau_{\gamma}$ along a curve $\gamma$ in $B$ with respect to the latter horizontal distribution acts as $\tau_{\gamma}([x, f])=\left[\tau_{\gamma}(x), f\right]$. Because $\partial F$ is invariant under $G$, we infer that parallel transport preserves $\partial X_{F}=X \times_{G} \partial F$ and the (MONODROMY) condition is satisfied. The (SYMPLECTIC FIBRATION) condition is satisfied by construction of $X_{F}$ and we are left to verify the (CONTACT TYPE BOUNDARY) condition. The natural Liouville vector field on $X \times F$ is

$$
\bar{Z}_{(x, \eta, f)}=\left(0, \beta, Z_{f}\right)
$$

By $G$-invariance $\bar{Z}$ descends to a Liouville vector field on $X_{F}$ provided it is tangent to $\phi^{-1}(0)$, and this is equivalent to $\phi_{F}(f)=d \phi_{F}(f) \cdot Z_{f}$ in a neighborhood of $\partial F$. Moreover, if the last condition holds, then verticality in $X_{F}$ is automatic.

Remark 3.9. Negative vector bundles, seen as associated bundles of the corresponding frame bundles, are a special instance of the above construction. 


\section{Fibered symplectic cohomology groups}

We define now the Floer or Symplectic cohomology groups $F H^{*}(E)$ for negative symplectic fibrations. The key concept is that of an asymptotically linear Hamiltonian, and from this point of view our definition can also be thought of as a bridge between the one of Viterbo [31], who uses Hamiltonians that are linear at infinity, and the one of Floer and Hofer [11], who use Hamiltonians that are asymptotically quadratic on $\mathbb{C}^{n}$ (with the somewhat surprising remark that "quadratic" is the same as "linear" after the change of variables $S=r^{2}$ ).

The main feature of the Floer cohomology groups that we define in this paper is that they have the same functorial properties as those of Viterbo in [31] (see Section 4.3).

Convention. We shall assume in this section that the form $\omega_{\epsilon}$ is symplectic for $0<\epsilon \leq 1$. For clarity, we drop the subscript $\epsilon$ and work with $\omega=\omega_{1}$.

4.1. Admissible Hamiltonians and almost complex structures. $C^{0}$ bounds. The crucial ingredient of the construction is the proof of a priori $C^{0}$-bounds for solutions $u: \mathbb{R} \times \mathbb{S}^{1} \longrightarrow \widehat{E}$ of the equation

$$
\begin{array}{r}
u_{s}+\widehat{J}(s, t, u)\left(u_{t}-X_{H}(s, t, u)\right)=0, \\
-\infty<\inf _{s \in \mathbb{R}} A_{H(s)}(u(s)), \sup _{s \in \mathbb{R}} A_{H(s)}(u(s))<+\infty .
\end{array}
$$

Here $H(s, t, u), \widehat{J}(s, t, u)$ is a homotopy of Hamiltonians and almost complex structures on which we impose additional constraints as described below. The constraints on $H$ and $\widehat{J}$, as well as the proofs of the $C^{0}$-estimates, are adapted from the papers of Cieliebak, Floer and Hofer [6, 11].

A point $u \in \widehat{E}$ which belongs to $\partial E \times[1, \infty[$ will be denoted $u=(\bar{u}, S)$.

Definition 4.1. Let $(\Sigma, \lambda)$ be a contact manifold. The manifold

$$
(\Sigma \times] 0, \infty[, d(S \lambda)), \quad S \in] 0, \infty[
$$

is called the symplectic cone over $\Sigma$, or the symplectization of $\Sigma$. The Reeb vector field $X_{\text {Reeb }}$ on $\Sigma$ is defined by $\iota_{X_{\text {Reeb }}} d \lambda \equiv 0, \lambda\left(X_{\text {Reeb }}\right)=1$. The contact distribution $\operatorname{ker} \lambda$ is denoted by $\xi$. An almost complex structure $J$ on $\Sigma \times] 0, \infty[$ is called standard if

$$
\begin{aligned}
J_{(\bar{u}, S)}\left(\frac{\partial}{\partial S}\right) & =\frac{1}{C S} X_{\text {Reeb }}(\bar{u}), \\
J_{(\bar{u}, S)}\left(X_{\text {Reeb }}(\bar{u})\right) & =-C S \frac{\partial}{\partial S}, \\
\left.J_{(\bar{u}, S)}\right|_{\xi} & =J_{0},
\end{aligned}
$$

where $J_{0}$ is an almost complex structure compatible with $d \lambda$ on $\xi$ and $C>0$ is a positive constant. 
Standard almost complex structures are precisely the ones that are preserved by homotheties in the $S$ variable. As an example, for $\Sigma=$ $\mathbb{S}^{2 n-1}(1) \subset \mathbb{C}^{n}$ and $\lambda=\frac{1}{2} \sum x_{i} d y_{i}-y_{i} d x_{i}$, the manifold $(\Sigma \times] 0, \infty[, d(S \lambda \mid))$ is symplectomorphic to $\left(\mathbb{C}^{n} \backslash\{0\}, \sum d x_{i} \wedge d y_{i}\right)$ through the map $(\bar{u}, S) \longmapsto$ $\varphi_{X}^{\ln S}(\bar{u})$. Here $\varphi_{X}^{t}$ stands for the flow of $X(z)=\frac{1}{2} z$. The inverse map satisfies $S(z)=\sqrt{|z|}$ and the canonical complex structure on $\mathbb{C}^{n} \backslash\{0\}$ translates into a standard almost complex structure on $\Sigma \times] 0, \infty[$ which satisfies (4.3) with $C=4$.

The metric $d(S \lambda)(\cdot, J \cdot)$ associated to a standard almost complex structure will be called conical. The following homogeneity property is straightforward:

$$
\left|v+a \frac{\partial}{\partial S}\right|_{(\bar{u}, S)}^{2}=S\left|v+\frac{a}{S} \frac{\partial}{\partial S}\right|_{(\bar{u}, 1)}^{2}, \quad v \in T_{\bar{u}} \Sigma, \quad a \in \mathbb{R} .
$$

Definition 4.2. Let $F \hookrightarrow E \stackrel{\pi}{\longrightarrow} B$ be a fibration satisfying the assumptions (SYMPLECTIC FIBRATION) and (CONTACT TYPE BOUNDARY). Let $H$ be the horizontal distribution on $\widehat{E}$. An almost complex structure $J$ on $\partial E \times[1, \infty[$ is called (standard) split if

$$
J=J_{V} \oplus \widetilde{J}_{B}
$$

where $J_{V}$ is a (standard) almost complex structure in the fibers and $\widetilde{J}_{B}$ is the lift to $H$ of an almost complex structure $J_{B}$ on $B$ which is $\beta$-tame.

The thrust of the present section is that the a priori $C^{0}$-bounds on Floer trajectories (and ultimately a variant of the maximum principle) hold in $\widehat{E}$ with respect to almost complex structures that are standard split at infinity.

Definition 4.3. An admissible homotopy of almost complex structures on $\widehat{E}$ is a smooth family $\widehat{J}(s, t), s \in \mathbb{R}, t \in \mathbb{S}^{1}$ of almost complex structures tamed by $\omega=\pi^{*} \beta+\Omega$ such that the following conditions hold.

i) $\widehat{J}$ is standard split for $S$ large enough, i.e., there exists $R \geq 1$ such that

$$
\widehat{J}(s, t, \bar{u}, S)=J_{V}(s, t, \bar{u}, S) \oplus \widetilde{J}_{B}(s, t, \bar{u}), \quad S \geq R .
$$

ii) $\widehat{J}$ is constant for $|s|$ large enough, i.e., there exists $s_{0}>0$ such that

$$
\begin{array}{llrl}
\widehat{J}(s, t, u) & =J_{-}(t, u), & & s \leq-s_{0}, \\
\widehat{J}(s, t, u) & =J_{+}(t, u), & & s \geq s_{0} .
\end{array}
$$

Here $J_{V}$ is a standard almost complex structure in the fiber and $\widetilde{J}_{B}$ is the horizontal lift of an almost complex structure $J_{B}$ on $B$ which is $\beta$-tame.

We now define admissible homotopies of Hamiltonians. In the usual setting of Floer homology, these are functions $H(s, t, \bar{u}, S), s \in \mathbb{R}, t \in \mathbb{S}^{1}$ with a 
special asymptotic behavior that ensures compactness for the moduli spaces of finite energy solutions of Floer's equation $u_{s}=-\nabla A_{H(s, \cdot)}(u(s, \cdot))$. Here

$$
A_{H(s, \cdot)}(x)=-\int_{D^{2}} \bar{x}^{*} \omega-\int_{\mathbb{S}^{1}} H(s) \circ x
$$

is the symplectic action defined on the space $\Lambda_{0} \widehat{E}$ of 1-periodic contractible loops in $\widehat{E}, \bar{x}$ denotes an extension of the loop $x$ over a disc and the $L^{2}$ gradient of $A_{H}$ is

$$
\nabla A_{H}(x)=\widehat{J} \dot{x}-\nabla H(x) .
$$

The construction of the spectral sequence will crucially require the use of negative pseudo-gradient trajectories for the action functional. We shall be interested in solutions $u(s, t)$ of

$$
\begin{gathered}
u_{s}=-\mathcal{Y}(u(s, \cdot)), \\
-\infty<\inf _{s \in \mathbb{R}} A_{H(s)}(u(s)), \quad \sup _{s \in \mathbb{R}} A_{H(s)}(u(s))<+\infty,
\end{gathered}
$$

where $\mathcal{Y}$ is a pseudo-gradient for some action functional $A_{H}$ on $\Lambda_{0} \widehat{E}$, i.e.,

$$
d A_{H}(x) \cdot \mathcal{Y}(x) \geq 0,
$$

with equality if and only if the loop $x$ is a critical point of $A_{H}$ (hence a periodic orbit of $X_{H}$ ). We shall actually need the stronger pseudo-gradient condition

$$
d A_{H}(x) \cdot \mathcal{Y}(x) \geq a^{2}\|\mathcal{Y}(x)\|^{2}, \quad a>0,
$$

with $\mathcal{Y}$ and $\nabla A_{H}$ having the same zeroes. We shall use vector fields $\mathcal{Y}$ of the type

$$
\mathcal{Y}(x)=\widehat{J} \dot{x}-Y(x),
$$

where $Y$ is a vector field on $\widehat{E}$. The vector field $Y$ will therefore be, along with the Hamiltonian $H$, part of the data defining an admissible deformation. In the following, we let $X=X^{\mathrm{v}}+X^{\mathrm{h}}$ be the decomposition of a vector $X \in T \widehat{E}$ in its vertical and horizontal parts.

Definition 4.4. Let $\widehat{J}$ be an admissible homotopy of almost complex structures. An admissible pseudo-gradient deformation consists of a one parameter family $H(s, t, u), s \in \mathbb{R}, t \in \mathbb{S}^{1}$ of Hamiltonians and of a one parameter family of vector fields $Y(s, t, u)$ on $\widehat{E}$, which satisfy the following properties.

i) (Strong pseudo-gradient) Let

$$
\mathcal{Y}(s, x)=\widehat{J}(s) \dot{x}-Y(s) \circ x .
$$

We require the existence of a function $a: \mathbb{R} \longrightarrow[0, \infty[$ with nowhere dense vanishing locus such that, for every loop $x \in \Lambda_{0} \widehat{E}$, we have

$$
d A_{H(s)}(x) \cdot \mathcal{Y}(s, x) \geq a(s)^{2}\|\mathcal{Y}(s, x)\|_{\widehat{J}(s)}^{2} .
$$


Moreover, $\mathcal{Y}(s, x)$ is required to have the same critical points as $\nabla A_{H(s)}$.

ii) (Monotonicity) $H$ is increasing

$$
\frac{\partial H}{\partial s}(s, t, u) \geq 0
$$

and there exists $s_{0}>0$ such that

$$
\begin{array}{lll}
H(s, t, u)=H_{-}(t, u), & Y(s, t, u)=Y_{-}(t, u), & a(s)=a_{-}>0, \\
H(s, t, u)=H_{+}(t, u), & Y(s, t, u)=Y_{+}(t, u), & a(s)=a_{+}>0,
\end{array}
$$

for $s \leq-s_{0}$, respectively $s \geq s_{0}$.

iii) (Asymptotes) There exists $f(s, \bar{u}): \mathbb{R} \times \partial E \longrightarrow] 1-\delta, \infty[$ so that the function $F(s, \bar{u}, S)=S f(s, \bar{u})$ satisfies

$$
\begin{array}{r}
\left|Y^{\mathrm{v}}-(\nabla F)^{\mathrm{v}}\right|_{J_{V}} / \sqrt{S} \longrightarrow 0, \\
|\nabla H-\nabla F|_{J_{V} \oplus \widetilde{J}_{B}} / \sqrt{S} \longrightarrow 0, \\
\left|\frac{\partial^{2} H}{\partial s \partial S}-\frac{\partial^{2} F}{\partial s \partial S}\right| \longrightarrow 0, \quad S \longrightarrow \infty,
\end{array}
$$

uniformly in $s, t$ and $\bar{u}$. Moreover, the function $f$ is required to satisfy the following conditions:

- For every $s \in \mathbb{R}$ and every large constant $c$, the horizontal distribution $H$ is tangent to

$$
(\text { graph } c / f(s)) \subset \partial E \times] 1-\delta, \infty[\text {. }
$$

- There exists $s_{0}>0$ such that

$$
\begin{aligned}
& f(s, \bar{u})=f^{-}(\bar{u}), \quad s \leq-s_{0}, \\
& f(s, \bar{u})=f^{+}(\bar{u}), \quad s \geq s_{0} .
\end{aligned}
$$

- The vector fields $X_{F^{ \pm}}$have no 1-periodic orbits at infinity, where

$$
F^{ \pm}(\bar{u}, S)=S f^{ \pm}(\bar{u}) .
$$

$$
\partial_{s} f \geq 0
$$

- If the 1-periodic orbits of $X_{F(\widehat{s})}$ are not contained in a compact set, then

$$
\left.\partial_{s} f\right|_{s=\widehat{s}} \geq \epsilon(\widehat{s})>0 .
$$

iv) (Boundedness) There exists a constant $c>0$ such that

$$
\begin{gathered}
\left|Y^{\mathrm{h}}\right|_{\beta, \widetilde{J}_{B}} \leq c \\
\left|\partial_{t} Y(s, t, \bar{u}, S)\right|_{J_{V} \oplus \widetilde{J}_{B}} \leq c(1+\sqrt{S}),
\end{gathered}
$$




$$
\left|\nabla_{X} Y(s, t, \bar{u}, S)\right|_{J_{V} \oplus \widetilde{J}_{B}} \leq c|X|_{J_{V} \oplus \widetilde{J}_{B}}, \quad X \in T_{(\bar{u}, S)}(\partial E \times[1, \infty[) .
$$

Remarks. 1. Condition (4.16) is equivalent to saying that the graph of $f(s)$ restricted to some fiber is a monodromy invariant hypersurface, and determines the values of $f(s)$ on the whole of $\partial E$ through parallel transport along $H$. This condition is clearly void if the base $B$ is a point. In case the base $B$ is not a point, it ensures that $X_{F(s)}, s \in \mathbb{R}$ is preserved by parallel transport along $H$, being colinear with the Reeb vector field on the level sets $c / f(s), c>>1$. We shall crucially use this fact in the proof of Proposition 4.8.

2. Condition (4.18) means that, for any choice of a large enough constant $c$, the graphs of $c / f^{ \pm}$have no closed characteristics of period 1. This can be achieved for example if $f^{ \pm}$are equal to constants not belonging to the period spectrum of $\partial E$ (this is a generic condition).

3 . The pseudo-gradient condition (4.10) obviously holds with $a(s) \equiv 1$ in case $\mathcal{Y}(s, x)=\nabla^{\widehat{J}(s)} A_{H(s)}(x)$, which corresponds to the usual Floer equation.

4. The function $F$ defined above satisfies

$$
\left|X_{F}\right|_{\omega}=O(\sqrt{S}) .
$$

5. The above conditions are satisfied in the $s$-independent case by vector fields $Y=\nabla h+\widetilde{\nabla f}$, where $h=h(S)$ is linear for $S$ big enough and $f$ : $B \longrightarrow \mathbb{R}$ is a smooth function. We must take in this case $F=h$ and $H=h+\widetilde{f}$. The pseudo-gradient property is the only nontrivial one, and we refer to Section 5 for a proof. There are two other nonempty properties, namely (4.21) which holds since $Y^{\mathrm{h}}=\widetilde{J}_{B} \widetilde{X}_{f}$, and (4.14) which holds because $\left|X_{\tilde{f}}\right| \widetilde{J}_{B}$ is bounded. This in turn is implied by the fact that $\left.\Omega\right|_{H_{0}}=\left.S d \Theta\right|_{H_{0}}$, hence the component of $X_{\widetilde{f}}$ on some nondegeneracy subspace of $\left.\Omega\right|_{H_{0}}$ goes to zero as $S \rightarrow \infty$, whereas the component on the degeneracy subspace stays bounded.

The proof of the $C^{0}$-bounds follows the arguments in $[\mathbf{1 1}]$. We recall the notations and a crucial technical result therein.

Let $\alpha: \mathbb{R} \times \mathbb{S}^{1} \longrightarrow \mathbb{R}$ be a smooth function. Let $\delta>0$. We denote by $\Gamma_{\delta}$ the set of all sequences $\left(s_{k}\right)_{k \in \mathbb{Z}}$ such that

$$
\begin{gathered}
0<s_{k+1}-s_{k} \leq \delta, \quad k \in \mathbb{Z}, \\
s_{k} \longrightarrow \pm \infty, \quad k \longrightarrow \pm \infty .
\end{gathered}
$$

For $s=\left(s_{k}\right) \in \Gamma_{\delta}$, we define

$$
[\alpha]^{s}=\sup \left\{\alpha\left(s_{k}, t\right): k \in \mathbb{Z}, t \in \mathbb{S}^{1}\right\} .
$$

Let

$$
[\alpha]_{\delta}=\inf \left\{[\alpha]^{s}: s \in \Gamma_{\delta}\right\}
$$


Proposition 4.5 ([11], Prop. 8). Let $A, B, \lambda \geq 0$ be nonnegative real numbers and let $\delta>0$ satisfy

$$
\delta^{2} \lambda<\pi^{2} .
$$

There exists a positive constant $C=C(A, B, \lambda, \delta)>0$ such that, for any function $\alpha: \mathbb{R} \times \mathbb{S}^{1} \longrightarrow[0, \infty[$ satisfying

$$
\begin{gathered}
-\Delta \alpha-\lambda \alpha \leq A \quad \text { on } \mathbb{R} \times \mathbb{S}^{1}, \\
{[\alpha]_{\delta}<B,}
\end{gathered}
$$

we have

$$
\sup \left\{\alpha(s, t):(s, t) \in \mathbb{R} \times \mathbb{S}^{1}\right\} \leq C .
$$

This is a key result involving the maximum principle, which is unavoidable in the proof of the $C^{0}$-bounds for all versions of Floer homology defined on open manifolds. Proposition 4.5 will be applied to the function $\alpha=S \circ u$, with $u$ an arbitrary solution of (4.7) and (4.8), and will ultimately yield the following result.

Theorem 4.6 (a priori $C^{0}$-bound; compare [11], Thm. 12). Let $\widehat{J}, H$ and $Y$ satisfy conditions (4.5) and (4.6) and (4.10) to (4.23). There is a constant $d=d(\widehat{J}, H, Y)>0$ such that any solution of

$$
\begin{array}{r}
u_{s}+\widehat{J}(s, t, u) u_{t}-Y(s, t, u)=0, \\
-\infty<\inf _{s \in \mathbb{R}} A_{H(s)}(u(s)), \quad \sup _{s \in \mathbb{R}} A_{H(s)}(u(s))<+\infty
\end{array}
$$

satisfies

$$
\sup _{(s, t) \in \mathbb{R} \times \mathbb{S}^{1}} S \circ u(s, t) \leq d .
$$

The rest of this section is devoted to the proof of Theorem 4.6.

\section{Notation.}

1a. We extend the function $\sqrt{S}$, which is canonically defined on $\partial E \times[1-$ $\delta, \infty[$, to a smooth function on the whole of $\widehat{E}$ as follows. Consider a strictly increasing smooth bijective map $\rho:[1-\delta, 1] \longrightarrow[0,1]$ whose derivatives vanish at infinite order at 0 and 1 . Our smooth extension of $\sqrt{S}$ is defined to be equal to $\rho(S) \sqrt{S}$ on $\partial E \times[1-\delta, \infty[$, and identically equal to zero on $\widehat{E} \backslash \partial E \times[1-\delta, \infty[$. We still denote this smooth extension by $\sqrt{S}$, and its square is a well-defined function on $\widehat{E}$, which we denote by $S$.

1b. Every point $u \in \partial E \times[1-\delta, \infty[$ can be uniquely written as $u=(\bar{u}, S)$, $\bar{u} \in \partial E \times\{1-\delta\}$. The map $u \longmapsto \bar{u}$ continuously extends as the identity over $E \backslash \partial E \times[1-\delta, 1]$ and we denote the extension again by $u \longmapsto \bar{u}$. 
1c. Every point $u \in \widehat{E}$ is now uniquely characterized by the pair $(\bar{u}, S)$ and we shall identify the two in the sequel.

2. We define the following three norms.

$$
\begin{aligned}
& |X|_{\beta}^{2}=\Omega\left(X^{\mathrm{v}}, J_{V} X^{\mathrm{v}}\right)+\pi^{*} \beta\left(X^{\mathrm{h}}, \widetilde{J}_{B} X^{\mathrm{h}}\right), \\
& |X|_{\omega}^{2}=\Omega\left(X^{\mathrm{v}}, J_{V} X^{\mathrm{v}}\right)+\omega\left(X^{\mathrm{h}}, \widetilde{J}_{B} X^{\mathrm{h}}\right), \\
& |X|_{\Omega}^{2}=\Omega\left(X^{\mathrm{v}}, J_{V} X^{\mathrm{v}}\right)+\Omega\left(X^{\mathrm{h}}, \widetilde{J}_{B} X^{\mathrm{h}}\right) .
\end{aligned}
$$

The last expression only defines $|\cdot|_{\Omega}$ as a semi-norm on $H$. Its utility will nevertheless become appearant in the sequel. The above norms all satisfy the homogeneity property (4.4) along the vertical distribution, whereas on the horizontal distribution, which is preserved by the Liouville flow, they satisfy the inequality

$$
\left|X^{\mathrm{h}}\right|_{(\bar{u}, S)}^{2} \leq S\left|X^{\mathrm{h}}\right|_{(\bar{u}, 1)}^{2}, \quad S \geq 1 .
$$

Convention. The default norm used in the sequel is $|\cdot|_{\omega}$.

3. We define

$$
\begin{aligned}
L^{2}\left(\mathbb{S}^{1}, \widehat{E}\right) & =\left\{x: \mathbb{S}^{1} \longrightarrow \widehat{E} \text { measurable }: \sqrt{S \circ x} \in L^{2}\left(\mathbb{S}^{1}, \mathbb{R}\right)\right\}, \\
H^{1}\left(\mathbb{S}^{1}, \widehat{E}\right) & =\left\{x \in L^{2}\left(\mathbb{S}^{1}, \widehat{E}\right): \dot{\bar{x}} \in L^{2}\left(x^{*} T E\right), \quad \sqrt{S \circ x} \in L^{2}\left(\mathbb{S}^{1}, \mathbb{R}\right)\right\} .
\end{aligned}
$$

Here $\widehat{E}$ is endowed with the metric $\langle X, Y\rangle_{\omega}=\frac{1}{2}(\omega(X, J Y)+\omega(Y, J X))$, $J=J_{V} \oplus \widetilde{J_{B}}$ and with its associated Lebesgue measure. The meaning of the condition $\dot{\bar{x}} \in L^{2}\left(x^{*} T E\right)$ is the following: $\dot{\bar{x}}$ is well defined as a distribution once we choose an embedding of $E$ into some Euclidean space. We require it to be an $L^{2}$-function, and this does not depend on the choice of the embedding.

Lemma 4.7. There is a compact embedding

$$
H^{1}\left(\mathbb{S}^{1}, \widehat{E}\right) \hookrightarrow C^{0}\left(\mathbb{S}^{1}, \widehat{E}\right) .
$$

Proof. Let $\left(y_{k}\right)$ be a sequence in $H^{1}\left(\mathbb{S}^{1}, \widehat{E}\right)$. We denote $S_{k}=S \circ y_{k}$. The embedding $H^{1}\left(\mathbb{S}^{1}, \mathbb{R}\right) \hookrightarrow C^{0}\left(\mathbb{S}^{1}, \mathbb{R}\right)$ is compact and therefore a subsequence of $\sqrt{S_{k}}$ converges uniformly to a continuous function $\sqrt{S_{0}}$. As a consequence, the corresponding subsequence of $y_{k}$ takes values in a compact set $\widehat{E}_{c}=$ $\{S \leq c\}$. The embedding $H^{1}\left(\mathbb{S}^{1}, \widehat{E}_{c}\right) \hookrightarrow C^{0}\left(\mathbb{S}^{1}, \widehat{E}_{c}\right)$ is again compact and we get a subsequence converging uniformly to a continuous limit $y$, with $S \circ y=S_{0}$.

Proposition 4.8 (compare [11], Lemma 10). Let $\widehat{J}, H$ and $Y$ satisfy conditions (4.5) and (4.6) and (4.10) to (4.23). Let $s \in \mathbb{R}$ be such that $a(s)>0$. For any choice of $c>0$ there is a constant $d=d(c, s)>0$ such that, if 
we have

$$
d A_{H(s)}(x) \cdot \mathcal{Y}(s, x)+\int_{0}^{1} \frac{\partial H}{\partial s}(s, t, x(t)) d t \leq c
$$

for some $x \in H^{1}\left(\mathbb{S}^{1}, \widehat{E}\right)$, then

$$
\|x\|_{H^{1}} \leq d
$$

We have denoted $\|x\|_{H^{1}}^{2}=\|\sqrt{S \circ x}\|_{H^{1}}^{2}+\|\dot{\bar{x}}\|_{L^{2}}^{2}$ for some fixed embedding of $E$ in a Euclidean space.

Remark 4.9. The statement is false if $a(s)$ is allowed to vanish. As a counterexample, one can consider a weak pseudo-gradient vector field of the form $Y=J_{V} X_{h}+\widetilde{J}_{B} \widetilde{X}_{f}$ as in Section 5 , where $h=h(S)$ has critical slope at infinity. The expression (4.27) is bounded if $x$ is a periodic orbit, but $x$ can nevertheless go to infinity.

Note also that, if $a(s) \geq a_{0}>0$ and $c \leq c_{0}<\infty$, then $d(c, s) \leq d\left(a_{0}, c_{0}\right)$.

Proof of Proposition 4.8. The strong pseudo-gradient condition (4.10) implies that $\|\mathcal{Y}(s, x)\|_{\widehat{J}(s)}^{2}$ is bounded by $c^{\prime}=c / a(s)^{2}$. The asymptotic behavior of $\widehat{J}$ ensures that the norms $|\cdot|_{\beta, \omega, \Omega}$ defined with respect to $\widehat{J}(s, t)$, $s \in \mathbb{R}, t \in \mathbb{S}^{1}$ are equivalent to the corresponding norms defined with respect to some fixed almost complex structure $J$ which is standard split at infinity. Moreover, the operator norm of $\widehat{J}$ is bounded. We can therefore assume in the sequel, without loss of generality, that $\widehat{J}=J$.

Claim 4.10. If $\|\mathcal{Y}(x)\|_{L^{2}}$ and $\|\sqrt{S \circ x}\|_{L_{2}}$ are bounded, then $\|x\|_{H^{1}}$ is bounded.

Proof of Claim 4.10. We remind that $\mathcal{Y}(x)=J \dot{x}-Y(x)$ and we have $|Y|_{\omega} \leq$ $\bar{c}_{1}(1+\sqrt{S})$. The hypothesis implies therefore that $\|\dot{x}\|_{L^{2}}$ is bounded.

Let $S(t)=S \circ x(t)$. At a point $t$ where $S(t) \geq 1$, we have $x(t)=(\bar{x}(t), S(t))$ and $|\dot{x}(t)|^{2}=|\dot{\bar{x}}(t)|_{x(t)}^{2}+\left|S^{\prime}(t) \frac{\partial}{\partial S}\right|^{2}=|\dot{\bar{x}}(t)|_{x(t)}^{2}+S^{\prime}(t)^{2} / S(t)$. This ensures $\sqrt{S}^{2} \leq \frac{1}{4}|\dot{x}(t)|^{2}$. At a point $t$ where $S(t) \leq 1$, we have $\sqrt{S}^{2}=[d(\sqrt{S}) \cdot \dot{x}(t)]^{2}$ $\leq c|\dot{x}(t)|^{2}$, the norm of $d \sqrt{S}$ being bounded on $E$.

On the other hand, we clearly have $|\dot{\bar{x}}(t)|_{\dot{\bar{x}}(t)} \leq|\dot{\bar{x}}(t)|_{x(t)} \leq|\dot{x}(t)|$ at a point $t$ where $S(t)>0$, while for $S(t)=0$ we have $\dot{\bar{x}}(t)=\dot{x}(t)$ hence $|\dot{\bar{x}}(t)|=|\dot{x}(t)|$.

This shows that $\|\dot{x}\|_{L^{2}}$ bounds $\left\|\sqrt{S}^{\prime}\right\|_{L^{2}}$ and $\|\dot{\bar{x}}\|_{L^{2}}$. Claim 4.10 is proved.

We are left to prove that $\|\sqrt{S \circ x}\|_{L^{2}}$ is bounded. Arguing by contradiction, let us suppose the existence of a sequence $\left(s_{k}, x_{k}\right)$ such that

$$
\left\|\sqrt{S \circ x_{k}}\right\| \longrightarrow \infty
$$




$$
\left\|\mathcal{Y}\left(s_{k}, x_{k}\right)\right\|^{2} \leq c^{\prime}, \quad \int_{0}^{1} \frac{\partial H}{\partial s}\left(s_{k}, t, x_{k}(t)\right) d t \leq c .
$$

By (4.12) we can suppose $s_{k} \longrightarrow \widehat{s}$. Let $S_{k}=S \circ x_{k}$ and $\lambda_{k}=\left\|\sqrt{S_{k}}\right\| / \Lambda$, with $\Lambda>1$ a constant to be chosen below.

Claim 4.11. The sequence $v_{k}(t)=\left(\bar{x}_{k}(t), S_{k}(t) / \lambda_{k}^{2}\right)$ has a uniformly convergent subsequence.

Proof of Claim 4.11. Let $\widetilde{S}_{k}=S \circ v_{k}$. We have $\left\|\sqrt{\widetilde{S}_{k}}\right\|=\Lambda<\infty$ and, by Lemma 4.7, it is enough to prove that $\left\|\dot{v}_{k}\right\|$ is bounded. We shall drop the subscript $k$ in the next paragraph. For $S>0$ we denote by $S^{o}\left(\operatorname{resp} . \widetilde{S}^{o}\right)$ the "true" $S$-coordinate corresponding to $S$ (resp. $\widetilde{S}$ ), with values in $] 1-\delta \infty[$ and with respect to which the homogeneity property (4.4) is verified.

We first prove the following inequality, with the convention $\widetilde{S}^{o} / S^{o}=1$ if $S=0$ :

$$
|\dot{v}(t)|^{2} \leq \bar{c} \frac{\widetilde{S}^{o}}{S^{o}}|\dot{x}|_{\Omega}^{2}+\left|\dot{\bar{x}}^{\mathrm{h}}\right|_{\beta}^{2}
$$

where $\bar{c} \geq 1$ is some constant. At a point $t$ where $S(t)=0$, we have $\dot{v}=\dot{x}$ and (4.30) is clear. Let us examine the situation at a point $t$ where $S(t)>0$. After expansion (4.30) reduces to

$$
\frac{\left(\widetilde{S}^{o \prime}\right)^{2}}{\widetilde{S}^{o}} \leq \bar{c} \frac{\widetilde{S}^{o}}{S^{o}} \frac{\left(S^{o \prime}\right)^{2}}{S^{o}},
$$

which is equivalent to

$$
\left|\frac{\widetilde{S}^{o \prime}}{\widetilde{S}^{o}}\right| \leq \bar{c}_{1}\left|\frac{S^{o l}}{S^{o}}\right|
$$

We prove (4.31) under the assumption that $\widetilde{S}^{o \prime}$ and $S^{o \prime}$ are both strictly positive (note that they necessarily have the same sign). The proof applies as such to the negative case as well.

We have $S^{o}=g(S)$ where $\left.g:\right] 0, \infty[\longrightarrow] 1-\delta, \infty[$ is a strictly increasing diffeomorphism, with $g(y)=y$ for $y \geq 1$ and $g^{\prime}\left(0^{+}\right)=\infty$. The inverse $f=g^{-1}$ has to vanish at infinite order at $1-\delta$ and has as typical profile $f(x)=\exp (-1 /(x-(1-\delta)))$. Therefore $g$ can be chosen to be equal to $g(y)=(1-\delta)-1 / \ln y$ near 0 , say on $] 0, \epsilon]$, with $g^{\prime}(y)=1 / y(\ln y)^{2}$, whereas on $[\epsilon, 1]$ we have $0<c \leq g^{\prime} \leq C$.

a. Assume $0<S \leq 1$. This means that $\widetilde{S}^{o}$ and $S^{o}$ belong to $] 1-\delta, 1$. It is enough to prove that, for any $\lambda \geq 1$, we have $g^{\prime}(S / \lambda) \leq \bar{c}_{2} \lambda g^{\prime}(S)$ for some constant $\bar{c}_{2}>0$. Now:

- If $S \in] 0, \epsilon]$, we have $g^{\prime}(S)=1 / S(\ln S)^{2}$ and $g^{\prime}(S / \lambda) \leq \lambda g^{\prime}(S)$.

- If $S \in] \epsilon, \epsilon \lambda]$, we have $g^{\prime}(S / \lambda) \leq \epsilon \lambda / S \cdot g^{\prime}(\epsilon) \leq \lambda C \leq \lambda C / c \cdot g^{\prime}(S)$.

- If $S \in] \epsilon \lambda, 1]$, we have $g^{\prime}(S / \lambda) \leq C / c \cdot g^{\prime}(S) \leq \lambda C / c \cdot g^{\prime}(S)$. 
b. Assume $S \geq 1$. We have to prove that $S / \lambda \cdot g^{\prime}(S / \lambda) \leq \bar{c}_{2} g(S / \lambda)$, $\lambda \geq 1$.

- If $1 \leq S \leq \epsilon \lambda$, then $S / \lambda \cdot g^{\prime}(S / \lambda) \leq 1 /(\ln \epsilon)^{2} \leq 1 /(1-$ $\delta)(\ln \epsilon)^{2} \cdot g(S / \lambda)$.

- If $1 \leq \epsilon \lambda \leq S \leq \lambda$, we have $S / \lambda \cdot g^{\prime}(S / \lambda) \leq 1 \cdot C \leq C /(1-\delta)$. $g(S / \lambda)$

- If $S \geq \lambda$, we have $g^{\prime}(S / \lambda)=1$ and $S / \lambda=g(S / \lambda)$.

Inequality (4.30) is now proved.

We can now proceed to the proof of Claim 4.11. We have

$$
\begin{aligned}
\|\dot{v}\|^{2}= & \int_{\widetilde{s}(t) \leq 1}|\dot{v}(t)|^{2} d t+\int_{\widetilde{s}(t)>1}|\dot{v}(t)|^{2} d t \\
\leq & 2 \int_{\widetilde{S}(t) \leq 1} \bar{c} \frac{\widetilde{S}^{o}}{S^{o}}|\dot{x}-J Y \circ x|_{\Omega}^{2}+\bar{c} \frac{\widetilde{S}^{o}}{S^{o}}|J Y \circ x|_{\Omega}^{2}+\left|\dot{\bar{x}}^{\mathrm{h}}-J Y \circ x^{\mathrm{h}}\right|_{\beta}^{2} \\
& +\left|J Y \circ x^{\mathrm{h}}\right|_{\beta}^{2}+2 \int_{\widetilde{s}(t)>1} \frac{1}{\lambda^{2}}|\dot{x}-J Y \circ x|_{\Omega}^{2}+\frac{1}{\lambda^{2}}|J Y \circ x|_{\Omega}^{2} \\
& +\left|\dot{\bar{x}}^{\mathrm{h}}-J Y \circ x^{\mathrm{h}}\right|_{\beta}^{2}+\left|J Y \circ x^{\mathrm{h}}\right|_{\beta}^{2} \\
\leq & 2\left(\bar{c}\|\dot{x}-J Y \circ x\|^{2}+\left\|J Y \circ x^{\mathrm{h}}\right\|_{\beta}^{2}\right. \\
& \left.+\int_{\widetilde{S}(t) \leq 1} \bar{c} \frac{\widetilde{S}^{o}|J Y \circ x|_{\Omega}^{2}}{S^{o}}+\int_{\widetilde{S}(t)>1} \frac{|J Y \circ x|_{\Omega}^{2}}{\lambda^{2}}\right) .
\end{aligned}
$$

We claim that all four terms in the last sum are bounded. The first equals $\|\mathcal{Y} \circ x\|$. The second is bounded by assumption (4.21) on $Y^{\mathrm{h}}$. In the third term, we have $\widetilde{S}^{o} \leq 1$ whereas $|J Y \circ x|_{\Omega}^{2} / S^{o}$ is bounded, as seen in the proof of Claim 4.10. Finally, the fourth term is bounded by $\frac{C}{\lambda^{2}}\|\sqrt{S \circ x}\|_{L^{2}}^{2}=C \Lambda$ and Claim 4.11 is proved.

After going to a subsequence, we can now assume that $v_{k} \stackrel{C^{0}}{\longrightarrow} v$ with $v$ continuous.

Claim 4.12. We have $S \circ v>1$ if $\Lambda$ is big enough.

We prove Claim 4.12 after having proved Claim 4.13 below.

Let now $U \subset B$ be a ball such that $\left.v \subset \widehat{E}\right|_{U}$, hence $\left.v_{k} \subset \widehat{E}\right|_{U}$ for $k$ large enough. The existence of $U$ follows from the contractibility of the $v_{k}$ 's. Choose a radial contraction of $U$ onto $b \in U$ and consider the associated parallel transport $\tau:\left.\widehat{E}\right|_{U} \longrightarrow \widehat{E}_{b}$. We define $\widetilde{v}=\tau \circ v, \widetilde{v}_{k}=\tau \circ v_{k}, \widetilde{x}_{k}=$ $\tau \circ x_{k}, \widetilde{\bar{x}}_{k}=\tau \circ \bar{x}_{k}$. We clearly have $\widetilde{x}_{k}=\left(\widetilde{\bar{x}}_{k}, S_{k}\right)$ and $\widetilde{v}_{k}=\left(\widetilde{\bar{x}}_{k}, S_{k} / \lambda_{k}^{2}\right)$.

Claim 4.13. We have $\left\|\dot{\widetilde{v}}_{k}-X_{F(\widehat{s})}(t, \widetilde{v})\right\|_{\Omega} \longrightarrow 0$.

Proof of Claim 4.13. We write in this paragraph $F$ for $F(\widehat{s})$ and we recall that, by condition (4.16), the vector field $X_{F}$ is vertical (and collinear to 
the Reeb vector field on the level surfaces of $F$ ). We have

$$
\left\|\dot{\widetilde{v}}_{k}-X_{F}(\widetilde{v})\right\|_{\Omega}=\frac{1}{\lambda_{k}}\left\|\dot{\widetilde{x}}_{k}-X_{F}\left(\lambda_{k}^{2} \widetilde{v}\right)\right\|_{\Omega},
$$

due to the homogeneity property of the conical metric. We also have

$$
\begin{aligned}
\frac{1}{\lambda_{k}}\left\|\dot{\widetilde{x}}_{k}-X_{F}\left(\lambda_{k}^{2} \widetilde{v}\right)\right\| \leq & \frac{1}{\lambda_{k}}\left(\left\|\dot{\widetilde{x}}_{k}-J Y^{\mathrm{vert}}\left(\widetilde{x}_{k}\right)\right\|+\left\|\left(J Y^{\mathrm{vert}}-X_{F}\right)\left(\widetilde{x}_{k}\right)\right\|\right) \\
& +\frac{1}{\lambda_{k}}\left\|X_{F}\left(\lambda_{k}^{2} \widetilde{v}_{k}\right)-X_{F}\left(\lambda_{k}^{2} \widetilde{v}\right)\right\| .
\end{aligned}
$$

The first term of the right hand side is bounded by $\frac{1}{\lambda_{k}}\|\mathcal{Y}(x)\|$ and goes to zero as $k$ goes to infinity. The second term goes to zero with $k$ by (4.13). The third term is bounded by $\left\|X_{F}\left(\widetilde{v}_{k}\right)-X_{F}(\widetilde{v})\right\|_{\Omega}$ and therefore also goes to zero. This proves Claim 4.13.

A direct consequence of Claim 4.13 is that $\dot{\widetilde{v}}=X_{F}(\widetilde{v})$. Indeed, we have $\dot{\tilde{v}}_{k} \stackrel{L^{2}}{\longrightarrow} X_{F}(\widetilde{v})$ and $\widetilde{v}_{k} \stackrel{L^{2}}{\longrightarrow} \widetilde{v}$. This implies $\dot{\widetilde{v}} \in L^{2}$ and $\dot{\widetilde{v}}=X_{F}(\widetilde{v})$.

Proof of Claim 4.12. We choose $\Lambda>1$ such that $\Lambda^{2} \cdot \min f(s, \bar{u})>$ $\max f(s, \bar{u})$. There exists $t_{0}$ such that $S\left(v\left(t_{0}\right)\right)=\Lambda^{2}$. Suppose by contradiction that $S(v(t))=1$ for some $t$ and let $t_{1}$ be the smallest such $t$ with $S \circ v>1$ on $\left[t_{0}, t_{1}\left[\right.\right.$ and $S\left(v\left(t_{1}\right)\right)=1$. The same argument as in the proof of Claim 4.13 can be applied on the interval $\left[t_{0}, t_{1}\right.$ [ instead of $\mathbb{S}^{1}$ in order to show that $\dot{\widetilde{v}}=X_{F}(\widetilde{v})$ on this interval. In particular, the image of $\left[t_{0}, t_{1}\right]$ under $\widetilde{v}$ is located on the level $S\left(\widetilde{v}\left(t_{0}\right)\right) f\left(\widehat{s}, \overline{\widetilde{v}}\left(t_{0}\right)\right)=\Lambda^{2} f\left(\widehat{s}, \overline{\widetilde{v}}\left(t_{0}\right)\right)>f\left(\widehat{s}, \overrightarrow{\widetilde{v}}\left(t_{1}\right)\right)$. In particular, $S\left(\widetilde{v}\left(t_{1}\right)\right) f\left(\widehat{s}, \overline{\widetilde{v}}\left(t_{1}\right)\right)>f\left(\widehat{s}, \overline{\widetilde{v}}\left(t_{1}\right)\right)$, which means $S\left(v\left(t_{1}\right)\right)>1$. This is a contradiction and Claim 4.12 is proved.

At this point, we have exhibited a 1-periodic orbit $\widetilde{v}$ for $X_{F}$, living on an arbitrarily large level. This ensures by (4.20) that $\frac{\partial f}{\partial s}(\widehat{s}) \geq \epsilon>0$. We shall put to work the hypothesis $\int_{0}^{1} \frac{\partial H}{\partial s} \leq c$ in order to derive a final contradiction and complete the proof of Proposition 4.8. We first compute:

$$
\begin{aligned}
& \frac{\partial H}{\partial s}(s, t, \bar{x}, S) \\
& =\int_{0}^{1} \frac{d}{d \gamma} \frac{\partial H}{\partial s}(s, t, \bar{x}, \gamma S+1-\gamma) d \gamma+\frac{\partial H}{\partial s}(s, t, \bar{x}, 1) \\
& =\int_{0}^{1} \frac{\partial^{2} H}{\partial s \partial S}(s, t, \bar{x}, \gamma S+1-\gamma) \cdot(S-1) d \gamma+\frac{\partial H}{\partial s}(s, t, \bar{x}, 1) \\
& =\int_{0}^{1}\left(\frac{\partial^{2} H}{\partial s \partial S}(s, t, \bar{x}, \gamma S+1-\gamma)-\frac{\partial^{2} F}{\partial s \partial S}(s, t, \bar{x}, \gamma S+1-\gamma)\right) \cdot(S-1) d \gamma \\
& \quad+\frac{\partial H}{\partial s}(s, t, \bar{x}, 1)+\partial_{s} f(s) \cdot(S-1) .
\end{aligned}
$$


We have used $\frac{\partial^{2} F}{\partial s \partial S}=\partial_{s} f$. On the other hand, condition (4.15) implies, for any $\tau>0$, the existence of a constant $c_{\tau}$ such that

$$
\left|\frac{\partial^{2} H}{\partial s \partial S}(s, t, \bar{x}, S)-\frac{\partial^{2} F}{\partial s \partial S}(s, \bar{x}, S)\right| \leq \tau+\frac{c_{\tau}}{\sqrt{S}} .
$$

For $k$ large enough, $s_{k}$ is close to $\widehat{s}$ and we get:

$$
\begin{aligned}
c & \geq \int_{0}^{1} \frac{\partial H}{\partial s}\left(s_{k}, t, \bar{x}_{k}(t), S_{k}(t)\right) d t \\
& \geq-\tau\left\|\sqrt{S_{k}}\right\|_{L^{2}}^{2}-c_{\tau}\left\|\sqrt{S_{k}}\right\|_{L^{1}}-C+\frac{\epsilon}{2}\left\|\sqrt{S_{k}}\right\|_{L^{2}}^{2}-\frac{\epsilon}{2} .
\end{aligned}
$$

But $\left\|\sqrt{S_{k}}\right\|_{L^{1}} \leq\left\|\sqrt{S_{k}}\right\|_{L^{2}}$ and, for $\tau<\epsilon$, the right hand term of the above inequality goes to $+\infty$ with $k$ because we have supposed $\left\|\sqrt{S_{k}}\right\|_{L^{2}} \longrightarrow \infty$. This is the desired contradiction and Proposition 4.8 is proved.

Lemma 4.14 (compare [11], Lemma 9). Let $\widehat{J}, H$ and $Y$ satisfy conditions (4.5) and (4.6) and (4.10) to (4.18). There exist $c_{1} \leq c_{2}$ such that every solution of

$$
\begin{array}{r}
u_{s}+\widehat{J}(s, t, u) u_{t}-Y(s, t, u)=0, \\
-\infty<\inf _{s \in \mathbb{R}} A_{H(s)}(u(s)), \quad \sup _{s \in \mathbb{R}} A_{H(s)}(u(s))<+\infty
\end{array}
$$

satisfies

$$
A_{H(s)} u(s) \in\left[c_{1}, c_{2}\right]
$$

Proof. We have

$$
\frac{d}{d s} A_{H(s)} u(s)=-\int_{0}^{1} \frac{\partial H}{\partial s}(s, t, u(s, t)) d t-d A_{H(s)}(u(s)) \cdot \mathcal{Y}(s, u(s)),
$$

where

$$
\|\xi\|_{s}^{2}=\int_{0}^{1} \omega(\xi(t), \widehat{J}(s, t) \xi(t)) d t, \quad x: \mathbb{S}^{1} \longrightarrow \widehat{E}, \quad \xi \in \Gamma\left(x^{*} T \widehat{E}\right) .
$$

By (4.11), we infer that $A_{H(s)} u(s)$ is decreasing with $s$ and therefore

$$
\begin{aligned}
& \lim _{s \rightarrow-\infty} A_{H(s)} u(s)=\sup _{s \in \mathbb{R}} A_{H(s)} u(s), \\
& \lim _{s \rightarrow+\infty} A_{H(s)} u(s)=\inf _{s \in \mathbb{R}} A_{H(s)} u(s) .
\end{aligned}
$$

We also have $\int_{-\infty}^{+\infty} d A_{H(s)}(u(s)) \cdot \mathcal{Y}(s, u(s))<\infty$ and we get a sequence $s_{k} \longrightarrow \infty$ such that $d A_{H\left(s_{k}\right)}\left(u\left(s_{k}\right)\right) \cdot \mathcal{Y}\left(s_{k}, u\left(s_{k}\right)\right) \longrightarrow 0$. We shall prove that $A_{H\left(s_{k}\right)} u\left(s_{k}\right)$ is bounded from below by a universal constant $c_{1}$. The same argument applied to a sequence $s_{k} \longrightarrow-\infty$ will yield the universal upper bound $c_{2}$.

We note at this point that, because $s_{k} \longrightarrow \infty$, we can assume by (4.6) and (4.12) that $\widehat{J}=J_{+}, H=H_{+}, Y=Y_{+}$are all independent of $s$. As 
usual, we denote $x_{k}=u\left(s_{k}\right), S_{k}=S \circ x_{k}$. The strong pseudo-gradient property holds therefore with the uniform constant $a_{+}>0$, and we infer that $\left\|\mathcal{Y}\left(s_{k}, x_{k}\right)\right\|_{s_{k}}^{2} \longrightarrow 0$.

Claim 4.15. $\left\|\sqrt{S_{k}}\right\|_{L^{2}}$ is bounded by a constant depending on $u$.

Let us assume for a moment that Claim 4.15 is true. Combining it with the fact that $\left\|\mathcal{Y}\left(x_{k}\right)\right\|_{L^{2}}$ is bounded we get by Claim 4.10 of Proposition 4.8 a $H^{1}$-bound on $x_{k}$ which again depends on $u$. By Lemma 4.7, we can then find a subsequence still denoted $x_{k}$ which converges uniformly to a continuous loop $x$.

We know that $\mathcal{Y}\left(x_{k}\right) \stackrel{L^{2}}{\longrightarrow} 0$ and therefore $x \in H^{1}, \mathcal{Y}(x)=0$ and $\dot{x}_{k} \stackrel{L^{2}}{\longrightarrow}$ $\dot{x}$. The fact that $\mathcal{Y}$ is a (negative) pseudo-gradient for the action implies that $x$ satisfies the equation $\dot{x}=X_{H_{+}}(x)$. By Proposition 4.8 applied to $H(s) \equiv H_{+}, \mathcal{Y}(s) \equiv \mathcal{Y}_{+}$, we get a universal bound on the $H^{1}$-norm of $x$. This implies a universal bound on its $C^{0}$-norm through Lemma 4.7 and a universal bound on its $C^{1}$-norm through the equation $\dot{x}=X_{H_{+}}(x)$. Finally, a $C^{1}$-bound on a contractible loop implies a universal bound on the action $A_{H_{+}}(x)$. Moreover, we have $A_{H\left(s_{k}\right)}\left(x_{k}\right) \longrightarrow A_{H_{+}}(x)$.

It is now clear that we can set

$$
c_{1}=\inf \left\{A_{H_{+}}(x): \dot{x}=X_{H_{+}}(x)\right\}>-\infty .
$$

Proof of Claim 4.15. We suppose by contradiction that, up to considering a subsequence, we have $\left\|\sqrt{S_{k}}\right\|_{L^{2}} \longrightarrow \infty$. We shall derive a contradiction along the lines of the proof of Proposition 4.8.

Let $\lambda_{k}=\left\|\sqrt{S_{k}}\right\|_{L^{2}} / \Lambda, \Lambda>1$ and $v_{k}(t)=\left(\bar{x}_{k}(t), S_{k}(t) / \lambda_{k}^{2}\right)$. We are now precisely in the situation of (4.29) in Proposition 4.8, with $H \equiv H_{+}$and $\mathcal{Y} \equiv$ $\mathcal{Y}_{+}$. We get a subsequence still denoted $v_{k}$ which converges to a continuous limit $v$, giving rise to a 1-periodic orbit of $X_{F_{+}}$which is located on a level set of $F_{+}$with arbitrarily large $S$ coordinate. This contradicts hypothesis (4.18) and concludes the proof of Claim 4.15 and of the Lemma.

Lemma 4.16 (compare [11], Proposition 11). Let $\widehat{J}, H$ and $Y$ satisfy conditions (4.5) and (4.6) and (4.10) to (4.23). For any $\delta>0$, there is a constant $c_{\delta}>0$ such that any solution of

$$
\begin{array}{r}
u_{s}+\widehat{J}(s, t, u) u_{t}-Y(s, t, u)=0, \\
-\infty<\inf _{s \in \mathbb{R}} A_{H(s)}(u(s)), \quad \sup _{s \in \mathbb{R}} A_{H(s)}(u(s))<+\infty
\end{array}
$$

satisfies

$$
[\sqrt{S \circ u}]_{\delta} \leq c_{\delta} .
$$

Proof. Let $u: \mathbb{R} \times \mathbb{S}^{1} \longrightarrow \widehat{E}$ satisfy the hypothesis. By Lemma 4.14 , there are constants $c_{1}<c_{2}$ such that $A_{H(s)} u(s) \in\left[c_{1}, c_{2}\right], s \in \mathbb{R}$. Let $\bar{c}=c_{2}-c_{1}$. 
We infer that, for any $a<b$, we have

$$
\begin{aligned}
& \int_{a}^{b}\left(d A_{H(s)}(u(s)) \cdot \mathcal{Y}(s, u(s))+\int_{0}^{1} \frac{\partial H}{\partial s}(s, t, u(s, t)) d t\right) d s \\
& \quad=A_{H(a)} u(a)-A_{H(b)} u(b) \leq \bar{c} .
\end{aligned}
$$

Let $\widehat{s}_{k}=k \delta / 4, \tau=\delta / 16$. Inequality (4.35) applied to $a=\widehat{s}_{k}-\tau$ and $b=\widehat{s}_{k}+\tau$, together with the density of the set $\{s: a(s)>0\}$, yields the existence of $s_{k} \in\left[\widehat{s}_{k}-\tau, \widehat{s}_{k}+\tau\right]$ such that $a\left(s_{k}\right)>0$ and such that $x_{k}=u\left(s_{k}\right)$ satisfies

$$
d A_{H\left(s_{k}\right)}\left(x_{k}\right) \cdot \mathcal{Y}\left(s_{k}, x_{k}\right)+\int_{0}^{1} \frac{\partial H}{\partial s}\left(s_{k}, t, x_{k}(t)\right) d t \leq \bar{c} \cdot 8 / \delta
$$

By the asymptotic behavior of $a(s)$, there are only a finite number of intervals on which $a(s)$ is nonconstant. We deduce the existence of some $a_{0}>0$ such that $a\left(s_{k}\right) \geq a_{0}$ for all $k \in \mathbb{Z}$. Proposition 4.8 implies the existence of a constant $d(\delta)$ such that $\left\|\sqrt{S \circ x_{k}}\right\|_{H^{1}}<d(\delta)$. The compact embedding $H^{1}\left(\mathbb{S}^{1}, \mathbb{R}\right) \hookrightarrow C^{0}\left(\mathbb{S}^{1}, \mathbb{R}\right)$ gives a constant $c_{\delta}$ such that $\left\|\sqrt{S \circ x_{k}}\right\|_{C^{0}} \leq c_{\delta}$. On the other hand, $\left(s_{k}\right) \in \Gamma_{\delta}$, where $\Gamma_{\delta}$ is defined by (4.24), and this implies $[\sqrt{S \circ u}]_{\delta} \leq c_{\delta}$.

Proof of Theorem 4.6. Let $\alpha(s, t)=S \circ u(s, t)$. By Lemma 4.16, we have $[\alpha]_{\delta} \leq\left(c_{\delta}\right)^{2}$ for any $\delta>0$. In view of Proposition 4.5, it is enough to show that $\alpha$ satisfies an equation of the form

$$
\Delta \alpha \geq-A-B \alpha
$$

with $A, B$ positive constants depending only on $\widehat{J}, H$ and $Y$ but not on $u$. Choosing $\delta$ such that $\delta^{2} B<\pi^{2}$ will yield a $C^{0}$-estimate on $\alpha$.

We first express $\Delta \alpha$ in a suitable way. The trick of exhibiting in (4.36) the term $\frac{1}{2}\left(\left|u_{s}\right|_{\Omega}^{2}+\left|u_{t}\right|_{\Omega}^{2}\right)$ is borrowed from [6]. Let us consider $R \geq 1 \mathrm{such}$ that $\widehat{J}(s, t)=J_{V}(s, t) \oplus \widetilde{J}_{B}(s, t)$ is standard split for $S \geq R^{2}$. Let $\Gamma=\{(s, t)$ : $\left.\alpha(s, t) \geq R^{2}\right\}$. We have $d S \circ \widehat{J}(s, t)=-C(s, t) S \Theta$ on $\Gamma$ and the following hold.

$$
\begin{aligned}
\alpha_{s}(s, t) & =d S \cdot u_{s}(s, t)=d S \cdot\left(-\widehat{J} u_{t}+Y(s, t, u)\right) \\
& =C(s, t) S(u(s, t)) \Theta\left(u_{t}\right)+d S \cdot Y(s, t, u) . \\
\alpha_{t}(s, t) & =d S \cdot u_{t}(s, t)=d S \cdot\left(\widehat{J} u_{s}-\widehat{J} Y(s, t, u)\right) \\
& =-C(s, t) S(u(s, t)) \Theta\left(u_{s}\right)+C(s, t) S(u(s, t)) \Theta(Y(s, t, u)) .
\end{aligned}
$$




$$
\begin{aligned}
\partial_{s} & \left.S(u(s, t)) \Theta\left(u_{t}\right)\right)-\partial_{t}\left(S(u(s, t)) \Theta\left(u_{s}\right)\right) \\
& =u_{s}\left((S \Theta)\left(u_{t}\right)\right)-u_{t}\left((S \Theta)\left(u_{s}\right)\right) \\
& =d(S \Theta)\left(u_{s}, u_{t}\right)+(S \Theta)\left(\left[u_{s}, u_{t}\right]\right) \\
& =d(S \Theta)\left(u_{s}, u_{t}\right)=\Omega\left(u_{s}, u_{t}\right)=\frac{1}{2} \Omega\left(u_{s}, u_{t}\right)+\frac{1}{2} \Omega\left(u_{s}, u_{t}\right) \\
& =\frac{1}{2} \Omega\left(u_{s}, \widehat{J} u_{s}-\widehat{J} Y\right)-\frac{1}{2} \Omega\left(u_{t},-\widehat{J} u_{t}+Y\right) \\
& =\frac{1}{2}\left(\left|u_{s}\right|_{\Omega}^{2}+\left|u_{t}\right|_{\Omega}^{2}\right)-\frac{1}{2} \Omega\left(u_{s}, \widehat{J} Y\right)-\frac{1}{2} \Omega\left(u_{t}, Y\right) .
\end{aligned}
$$

We get

$$
\begin{aligned}
\Delta \alpha= & \frac{C(s, t)}{2}\left(\left|u_{s}\right|_{\Omega}^{2}+\left|u_{t}\right|_{\Omega}^{2}\right)-\frac{C(s, t)}{2}\left(\Omega\left(u_{s}, \widehat{J} Y\right)+\Omega\left(u_{t}, Y\right)\right) \\
& +C_{s}(s, t) S \Theta\left(u_{t}\right)-C_{t}(s, t) S \Theta\left(u_{s}\right)+C_{t}(s, t) S \Theta(Y) \\
& +d S \cdot \partial_{s} Y(s, t, u)+d S \cdot \nabla_{u_{s}} Y(s, t, u) \\
& +C(s, t)\left(\left(d S \cdot u_{t}\right) \cdot \Theta(Y)+S \Theta\left(\partial_{t} Y(s, t, u)\right)+S \Theta\left(\nabla_{u_{t}} Y(s, t, u)\right)\right) .
\end{aligned}
$$

We estimate now the terms composing the right side of the above identity. Condition (4.6) implies that $C(s, t)$ is independent of $s$ for $|s| \geq s_{0}$. As $C(s, t)>0$ we get the existence of strictly positive constants $C_{0}, C_{1}$ such that

We get

$$
0<C_{0} \leq C(\cdot, \cdot) \leq C_{1}, \quad|d C(\cdot, \cdot)| \leq C_{1}
$$

$$
\frac{C(s, t)}{2}\left(\left|u_{s}\right|_{\Omega}^{2}+\left|u_{t}\right|_{\Omega}^{2}\right) \geq \frac{C_{0}}{2}\left(\left|u_{s}\right|_{\Omega}^{2}+\left|u_{t}\right|_{\Omega}^{2}\right) .
$$

On the other hand, we have

$$
\left|\Omega\left(u_{s}, \widehat{J} Y\right)\right| \leq\left|u_{s}\right|_{\Omega} \cdot|Y|_{\Omega}, \quad\left|\Omega\left(u_{t}, Y\right)\right| \leq\left|u_{t}\right|_{\Omega} \cdot|Y|_{\Omega} .
$$

We can estimate $|Y|_{\Omega}$ through the first condition of (4.13), which implies $|Y|_{\Omega} \leq|Y|_{\omega} \leq c_{1}(1+\sqrt{S})+\left|X_{F}\right|_{\omega}$. Now a direct computation shows that $\left|X_{F}\right|_{\omega}=O(\sqrt{S})$ and this gives $|Y|_{\Omega} \leq \bar{c}_{1}(1+\sqrt{S})$. As a consequence, we have

$$
\begin{aligned}
\left|\Omega\left(u_{s}, \widehat{J} Y\right)\right| & \leq \bar{c}_{1}(1+\sqrt{\alpha})\left|u_{s}\right|_{\Omega}, \\
\left|\Omega\left(u_{t}, Y\right)\right| & \leq \bar{c}_{1}(1+\sqrt{\alpha})\left|u_{t}\right|_{\Omega} .
\end{aligned}
$$

The norm of $\Theta(\bar{u}, S)$ as a linear map is equal to $1 / \sqrt{S}$ for $S \geq 1$, and we get

$$
\begin{gathered}
\left|C_{s} S \Theta\left(u_{t}\right)\right| \leq C_{1}(1+\sqrt{\alpha})\left|u_{t}\right|, \\
\left|C_{t} S \Theta\left(u_{s}\right)\right| \leq C_{1}(1+\sqrt{\alpha})\left|u_{s}\right|, \\
\left|C_{t} S \Theta(Y)\right| \leq C_{1} \bar{c}_{1}(1+\sqrt{\alpha})^{2} .
\end{gathered}
$$


The norm of $d S(\bar{u}, S)$ as a linear map is equal to $\sqrt{S}$ for $S \geq 1$, while $\left|\partial_{s} \nabla F(s)\right|_{\omega}=O(\sqrt{S})$. The second condition in (4.13) implies $\left|\partial_{s} Y\right|_{\omega}=$ $O(\sqrt{S})$ and we obtain

$$
\left|d S \cdot \partial_{s} Y(s, t, u)\right| \leq c_{2}(1+\sqrt{\alpha})^{2} .
$$

We have as well

$$
\left|\left(d S \cdot u_{t}\right) \cdot \Theta(Y(s, t, u))\right| \leq \bar{c}_{1}(1+\sqrt{\alpha})\left|u_{t}\right|_{\Omega}
$$

and, by (4.22):

$$
\left|S \Theta\left(\partial_{t} Y(s, t, u)\right)\right| \leq c(1+\sqrt{\alpha})^{2} .
$$

Finally, condition (4.23) gives

$$
\begin{aligned}
\left|S \Theta\left(\nabla_{u_{t}} Y(s, t, u)\right)\right| & \leq c(1+\sqrt{\alpha})\left|u_{t}\right|_{\Omega}, \\
\left|d S \cdot \nabla_{u_{s}} Y(s, t, u)\right| & \leq c(1+\sqrt{\alpha})\left|u_{s}\right|_{\Omega} .
\end{aligned}
$$

The absolute value of the sum of the terms other than $\frac{1}{2} C(s, t)\left(\left|u_{s}\right|_{\Omega}^{2}+\right.$ $\left.\left|u_{t}\right|_{\Omega}^{2}\right)$ is therefore bounded by

$$
\bar{C}_{1}(1+\alpha)+\bar{C}_{2}(1+\sqrt{\alpha})\left|u_{t}\right|_{\Omega}+\bar{C}_{3}(1+\sqrt{\alpha})\left|u_{s}\right|_{\Omega},
$$

with obvious constants $\bar{C}_{1}, \bar{C}_{2}$ and $\bar{C}_{3}$. This implies

$$
\Delta \alpha \geq-A-B \alpha,
$$

with suitable constants $A$ and $B$. This inequality holds for $\alpha(s, t) \geq R^{2}$. In order to get a global inequality on $\mathbb{R} \times \mathbb{S}^{1}$, we use a trick that we borrow from [11]. Let $\varphi: \mathbb{R}_{+} \longrightarrow \mathbb{R}_{+}$be a smooth function such that $\varphi(S) \equiv 0$ for $S \leq R^{2}, \varphi^{\prime}(S)=1$ for $S \geq R^{2}+1$ and $\varphi^{\prime \prime}(S)>0$ for $R^{2}<S<R^{2}+1$. Then $\varphi$ satisfies

$$
S \leq \varphi(S)+\bar{C}
$$

for a suitable constant $\bar{C}$. Let $\beta(s, t)=\varphi \circ \alpha(s, t)$. Inequality (4.37) gives a bound on $\alpha$ in terms of a bound on $\beta$. On the other hand, we obviously have $[\beta]_{\delta} \leq[\alpha]_{\delta}, \delta>0$. It is therefore enough to show that $\beta$ satisfies an inequality of the form $\Delta \beta \geq-A^{\prime}-B^{\prime} \beta$, with $A^{\prime} \geq 0, B^{\prime} \geq 0$ positive constants. Indeed

$$
\begin{aligned}
\Delta \beta & =\partial_{s}\left(\varphi^{\prime}(\alpha(s, t)) \cdot \alpha_{s}\right)+\partial_{t}\left(\varphi^{\prime}(\alpha(s, t)) \cdot \alpha_{t}\right) \\
& =\varphi^{\prime \prime}(\alpha(s, t)) \cdot\left(\left(\alpha_{s}\right)^{2}+\left(\alpha_{t}\right)^{2}\right)+\varphi^{\prime}(\alpha(s, t)) \cdot \Delta \alpha \\
& \geq \varphi^{\prime}(\alpha(s, t))(-A-B \alpha) \geq-A-B \alpha \geq-A-B \beta .
\end{aligned}
$$

Remark 4.17 (On the maximum principle). A priori $C^{0}$-bounds for Hamiltonians that are linear at infinity can be obtained directly through the maximum principle as in [31]. Nevertheless, the interested reader can convince himself that such a direct approach is not effective for Hamiltonians of the form $h(S)+\widetilde{f}$. The solutions of Floer's equation satisfy in this case 
a second-order elliptic equation with zero-order term - an avatar of which has already appeared in the previous proof - and no general maximum principle is available in this context.

4.2. Definition of symplectic cohomology. We define in this mainly expository section the Symplectic cohomology groups $F H_{[a, b]}^{*}(E)$. We combine the philosophy of $[\mathbf{3 1}]$ with the setup of $[\mathbf{1 1}]$.

Let $\mathcal{J}(\widehat{E})$ be the set of admissible almost complex structures, consisting of time-dependent elements $\widehat{J}$ such that the associated constant deformation $\widehat{J}(s) \equiv \widehat{J}$ satisfies $(4.5)$ in Definition 4.3 . Let $\mathcal{J}(\mathbb{R} ; \widehat{E})$ be the set of admissible deformations of almost complex structures given by Definition 4.3.

Given $\widehat{J} \in \mathcal{J}(\widehat{E})$, we denote by $\mathcal{H} \mathcal{Y}(E, \widehat{J})$ the set of admissible Hamiltonians and pseudo-gradient vector fields, consisting of time-dependent pairs $(H, Y)$ such that the constant deformation $(H(s), Y(s)) \equiv(H, Y)$ satisfies Definition 4.4 and $\left.H\right|_{\mathbb{S}^{1} \times E}<0$. Given $\widehat{J} \in \mathcal{J}(\mathbb{R} ; \widehat{E})$, we denote by $\mathcal{H} \mathcal{Y}(\mathbb{R} ; E, \widehat{J})$ the set of admissible deformations of Hamiltonians and pseudogradient vector fields, consisting of pairs $(H, Y)$ satisfying Definition 4.4 and such that $\left.H(s)\right|_{\mathbb{S}^{1} \times E}<0, s \in \mathbb{R}$.

We also define

$$
\mathcal{J H} \mathcal{Y}(E)=\bigcup_{\widehat{J}}\{\widehat{J}\} \times \mathcal{H} \mathcal{Y}(E, \widehat{J}), \quad \mathcal{J} \mathcal{H} \mathcal{Y}(\mathbb{R} ; E)=\bigcup_{\widehat{J}}\{\widehat{J}\} \times \mathcal{H} \mathcal{Y}(\mathbb{R} ; E, \widehat{J}) .
$$

Definition 4.18. Let $p>2$ be fixed. We define $\mathcal{J} \mathcal{H} \mathcal{Y}_{\text {reg }}(E)$ to be the set of all triples $(\widehat{J}, H, Y) \in \mathcal{J} \mathcal{H} \mathcal{Y}(E)$ such that the 1-periodic orbits of $H$ are nondegenerate and the linearized operator

$$
\begin{gathered}
D_{u}: W^{1, p}\left(u^{*} T \widehat{E}\right) \longrightarrow L^{p}\left(u^{*} T \widehat{E}\right), \\
D_{u} \xi=\nabla_{s} \xi+\widehat{J}_{t}(u) \nabla_{s} \xi+\nabla_{\xi} J_{t}(u) \partial_{t} u-\nabla_{\xi} Y_{t}(u)
\end{gathered}
$$

is surjective for any finite energy solution $u: \mathbb{R} \times \mathbb{S}^{1} \longrightarrow \widehat{E}$ of the equation

$$
u_{s}+\widehat{J}(t, u(s, t)) u_{t}=Y(t, u(s, t)) .
$$

It is proved in $[\mathbf{9}, \mathbf{2 7}]$ that, if the 1-periodic orbits of $H$ are nondegenerate, the operator $D_{u}$ is Fredholm and its index at a solution $u$ is equal to

$$
\text { ind } D_{u}=i_{\mathrm{CZ}}\left(x^{+}\right)-i_{C Z}\left(x^{-}\right), \quad x^{ \pm}=\lim _{s \rightarrow \pm \infty} u(s, \cdot) .
$$

We have denoted by $i_{\mathrm{CZ}}$ the Conley-Zehnder index as defined in [26]. The arguments in $[\mathbf{1 2}]$ can be adapted to the pseudo-gradient setting and show that $\mathcal{J H} \mathcal{Y}_{\text {reg }}(E)$ is of the second Baire category in $\mathcal{J H} \mathcal{Y}(E)$. 
Let $\mathcal{P}(H)$ be the set of 1-periodic orbits of $H$. Given a regular triple $(\widehat{J}, H, Y)$, we define the complex

$$
\begin{aligned}
F C^{*}(\widehat{J}, H, Y) & =\bigoplus_{\substack{x \in \mathcal{P}(H) \\
-i_{\mathrm{CZ}}(x)=*}} \mathbb{Z}\langle x\rangle, \\
\partial\langle x\rangle & =\sum_{y: \operatorname{dim} \mathcal{M}(y, x)=1} \#(\mathcal{M}(y, x) / \mathbb{R})\langle y\rangle .
\end{aligned}
$$

Here $\mathcal{M}(y, x)$ stands for the space of solutions $u$ of equation (4.38) with the limit conditions $\lim _{s \rightarrow-\infty} u(s, \cdot)=y(\cdot), \lim _{s \rightarrow \infty} u(s, \cdot)=x(\cdot)$. The additive group $\mathbb{R}$ acts on $\mathcal{M}(y, x)$ by reparametrizations, and we denote by $\#(\mathcal{M}(y, x) / \mathbb{R})$ the algebraic number of elements of $\mathcal{M}(y, x) / \mathbb{R}$ with respect to a choice of coherent orientations $[\mathbf{1 0}]$. We claim that $\partial^{2}=0$ and hence $\left(F C^{*}(\widehat{J}, H, Y), \partial\right)$ is a differential complex.

The first important observation is that the equation $u_{s}+\widehat{J} u_{t}=$ $Y(t, u(s, t))$ has the same analytic nature as the ordinary Floer equation, namely the linearization $D_{u}$ is a compact perturbation of the CauchyRiemann operator. In particular, a bound on the energy $E(u)=\int_{\mathbb{R} \times \mathbb{S}^{1}}\left|u_{s}\right|^{2}$ implies compactness up to breaking of trajectories for the relevant moduli spaces. This in turn implies $\partial^{2}=0$. The second observation is that such a uniform bound on the energy for the elements of $\mathcal{M}(y, x)$ follows from the strong pseudo-gradient property $d A_{H} \cdot \mathcal{Y} \geq c\|\mathcal{Y}\|^{2}, c>0$. Indeed, if $u_{s}=-\mathcal{Y}(t, u(s, \cdot))$ and $u(-\infty, \cdot)=y(\cdot), u(+\infty, \cdot)=x(\cdot)$, we get

$$
A_{H}(y)-A_{H}(x)=-\int_{\mathbb{R}} \frac{d}{d s} A_{H}(u(s, \cdot))=\int_{\mathbb{R}} d A_{H} \cdot \mathcal{Y} \geq c \int_{\mathbb{R}}\|\mathcal{Y}\|^{2}=c E(u),
$$

so that $E(u) \leq\left(A_{H}(y)-A_{H}(x)\right) / c$.

Remark 4.19 (Convexity). Since the space of strong pseudo-gradient vector fields is convex, the homology groups that are computed by means of any strong pseudo-gradient are the same as the ones computed with the usual gradient of the action functional.

The pseudo-gradient property implies that the action decreases along solutions of (4.38), so that we have subcomplexes

$$
F C_{[a, \infty[}^{*}(\widehat{J}, H, Y)=\bigoplus_{A_{H}(x)>a} \mathbb{Z}\langle x\rangle \subset F C^{*}(\widehat{J}, H, Y)
$$

defined for $a \leq \infty$, as well as quotient complexes

$$
F C_{[a, b]}^{*}(\widehat{J}, H, Y)=F C_{[a, \infty[}^{*} / F C_{[b, \infty[}^{*}=\bigoplus_{\left.\left.A_{H}(x) \in\right] a, b\right]} \mathbb{Z}\langle x\rangle
$$


defined for $-\infty \leq a<b \leq \infty$. We denote the corresponding homology groups by $F H_{[a, b]}^{*}(\widehat{J}, H, Y)$. They are endowed with natural restriction morphisms induced by inclusions of subcomplexes

$$
F H_{[a, b]}^{*}(\widehat{J}, H, Y) \longrightarrow F H_{\left[a^{\prime}, b^{\prime}\right]}^{*}(\widehat{J}, H, Y), \quad a \geq a^{\prime}, b \geq b^{\prime} .
$$

The above homology groups depend on the asymptotic profile $F$ of the Hamiltonian $H$ (see Definition 4.4). In order to define invariants of $\widehat{E}$, one needs to use an algebraic limit procedure which we describe below, and for which we need to further restrict the class of admissible deformations considered in Definition 4.4 in order to be able to get a priori energy bounds for solutions of the $s$-dependent Floer equation.

Definition 4.20. Let $\widehat{J} \in \mathcal{J}(\mathbb{R} ; \widehat{E})$. A good deformation of Hamiltonians and pseudo-gradient vector fields is an admissible deformation $(H, Y)$ such that $\left.H(s)\right|_{\mathbb{S}^{1} \times E}<0, s \in \mathbb{R}$ and the following additional condition is satisfied.

- For any compact set $K \subset \widehat{E}$, there exists a constant $a_{K}>0$ such that

$$
d A_{H(s, \cdot)}(x) \cdot \mathcal{Y}(s, x) \geq a_{K}\|\mathcal{Y}(s, x)\|_{\widehat{J}(s)}^{2}
$$

for all contractible loops $x: \mathbb{S}^{1} \rightarrow K$ and all $s \in \mathbb{R}$.

Remark 4.21 (admissible versus good deformations). The difference between admissible and good deformations is that, for the latter, $\mathcal{Y}(s, \cdot)$ might still be only a weak pseudo-gradient for a nowhere dense set of values $s \in \mathbb{R}$, but this phenomenon is controlled in a precise way, namely $a_{K} \rightarrow 0$ as $K$ exhausts $\widehat{E}$.

Remark 4.22 (Example). Given two triples $T_{ \pm}=\left(\widehat{J}_{ \pm}, H_{ \pm}, Y_{ \pm}\right) \in \mathcal{J H} \mathcal{H}(E)$, we can construct a good deformation connecting them as follows. We interpolate from $Y_{-}$to $\nabla^{\widehat{J}_{-}} H_{-}$, then deform $\widehat{J}_{-}$to $\widehat{J}_{+}, H_{-}$to $H_{+}$and implicitly $\nabla^{\widehat{J}_{-}} H_{-}$to $\nabla^{\widehat{J}_{+}} H_{+}$, and then interpolate from $\nabla^{\widehat{J}^{+}} H_{+}$to $Y_{+}$. Such deformations admit a uniform pseudo-gradient constant $a>0$ depending only on $T_{ \pm}$.

Remark 4.23 (Degree of generality). We actually use in Section 7.6 good deformations which satisfy (4.43) with a uniform constant, independent of the compact set $K$. Nevertheless, we chose to give the more general Definition 4.20 in order to stress the fundamental role of the strong pseudogradient inequality.

We denote by

$$
\mathcal{J H} \mathcal{Y}\left(E ; T_{-}, T_{+}\right) \subset \mathcal{J H} \mathcal{Y}(\mathbb{R} ; E)
$$

the space of good deformations connecting $T_{-}$and $T_{+}$. Standard transversality methods allow one to define a space

$$
\mathcal{J H} \mathcal{Y}_{\text {reg }}\left(E ; T_{-}, T_{+}\right)
$$


of regular good deformations, which is a set of second Baire category in $\mathcal{J H} \mathcal{Y}\left(E ; T_{-}, T_{+}\right)$and has the property that the spaces of solutions of the equation

$$
u_{s}+\widehat{J}(s, t, u(s, t)) u_{t}=Y(s, t, u(s, t))
$$

are smooth manifolds. We claim that, just like in the case of constant deformations, these spaces of solutions are compact modulo breaking of trajectories. The main ingredient is an a priori energy bound for the elements of the space $\mathcal{M}\left(x^{-}, x^{+}\right)$of solutions connecting $x^{-} \in \mathcal{P}\left(H_{-}\right)$to $x^{+} \in \mathcal{P}\left(H_{+}\right)$, and Definition 4.20 plays a crucial role in obtaining it. Since a good deformation is admissible, we know that Floer trajectories stay inside a compact set $K$, hence $\mathcal{Y}$ is a strong pseudo-gradient along Floer trajectories with uniform pseudo-gradient constant $a_{K}>0$ and we have

$$
\begin{aligned}
A_{H_{-}}\left(x^{-}\right)-A_{H_{+}}\left(x^{+}\right) & =-\int_{\mathbb{R}} \frac{d}{d s} A_{H(s)}(u(s, \cdot))=\int_{\mathbb{R}} d A_{H(s)} \cdot \mathcal{Y}+\int_{\mathbb{R} \times \mathbb{S}^{1}} \frac{\partial H}{\partial s} \\
& \geq a_{K} \int_{\mathbb{R}}\|\mathcal{Y}\|^{2}=a_{K} E(u) .
\end{aligned}
$$

Therefore, $E(u) \leq\left(A_{H_{-}}\left(x^{-}\right)-A_{H_{+}}\left(x^{+}\right)\right) / a_{K}$ is a priori bounded. Note that, although we have used the hypothesis $\partial H / \partial s \geq 0$ in the above computation, this is not a crucial assumption at this point.

The pseudo-gradient property again implies that the symplectic action decreases along solutions of (4.44), hence a good deformation induces chain maps

$$
\sigma: F C_{[a, \infty[}^{*}\left(\widehat{J}_{+}, H_{+}, Y_{+}\right) \longrightarrow F C_{[a, \infty[}^{*}\left(\widehat{J}_{-}, H_{-}, Y_{-}\right)
$$

which pass to the quotient as chain maps

$$
\sigma: F C_{[a, b]}^{*}\left(\widehat{J}_{+}, H_{+}, Y_{+}\right) \longrightarrow F C_{[a, b]}^{*}\left(\widehat{J}_{-}, H_{-}, Y_{-}\right), \quad-\infty \leq a<b \leq \infty .
$$

Since the space of good deformations is convex, the induced morphisms in homology

$$
\sigma: F H_{[a, b]}^{*}\left(\widehat{J}_{+}, H_{+}, Y_{+}\right) \longrightarrow F H_{[a, b]}^{*}\left(\widehat{J}_{-}, H_{-}, Y_{-}\right), \quad-\infty \leq a<b \leq \infty
$$


do not depend on the choice of the deformation. We call them monotonicity morphisms. We define the Floer or symplectic cohomology groups by

$$
\begin{aligned}
F H_{[a, b]}^{*}(E) & =\lim _{\longleftarrow} F H_{[a, b]}^{*}(\widehat{J}, H, Y), \\
F H^{*}(E) & =\lim _{\longleftarrow} F H_{[-\infty, b]}^{*}(E) .
\end{aligned}
$$

Here the partial order $\prec$ on $\mathcal{J H} \mathcal{Y}(E)$ is defined by

$$
\left(J_{-}, H_{-}, Y_{-}\right) \prec\left(J_{+}, H_{+}, Y_{+}\right) \quad \text { iff } H_{-}(t, x) \leq H_{+}(t, x), \quad t \in \mathbb{S}^{1}, x \in \widehat{E},
$$

and it makes $\mathcal{J H} \mathcal{Y}(E)$ into a directed set.

We conclude this section with an invariance statement.

Theorem 4.24 ([4], Lemma 3.7, [31], Theorem 1.7). Let $\beta_{t}, \Omega_{t}, Z_{t}, t \in$ $[0,1]$ be a deformation of the symplectic structure on the fibration $E$ such that

$$
\left(E, \pi, B, F, \Omega_{t}, Z_{t}, \beta_{t}\right)
$$

defines a negative symplectic fibration in the symplectically aspherical category for each $t \in[0,1]$. We then have a natural isomorphism

$$
F H^{*}\left(E ; \beta_{0}, \Omega_{0}, Z_{0}\right) \simeq F H^{*}\left(E ; \beta_{1}, \Omega_{1}, Z_{1}\right) .
$$

4.3. Properties of symplectic cohomology. In this section, all symplectic manifolds are assumed to be symplectically aspherical. We recall the definition of positive contact type boundary given in the Introduction.

Definition 4.25. Let $(M, \omega)$ be a symplectically aspherical manifold with boundary of contact type and Liouville form $\lambda$ defined in a neighborhood of $\partial M$. We say that $\partial M$ is of positive contact type if every positively oriented closed contractible characteristic $\gamma$ has positive action $A_{\omega}(\gamma)$ bounded away from zero, i.e., there exists $T_{0}>0$ such that

$$
A_{\omega}(\gamma, \bar{\gamma})=A_{\omega}(\gamma):=\int_{D^{2}} \bar{\gamma}^{*} \omega \geq T_{0}
$$

where $\bar{\gamma}: D^{2} \longrightarrow M$ is any map satisfying $\left.\bar{\gamma}\right|_{\partial D^{2}}=\gamma$.

Remark 4.26 (Examples). 1. Restricted contact type implies positive contact type in view of the equality $\int_{D^{2}} \bar{\gamma}^{*} \omega=\int_{\mathbb{S}^{1}} \gamma^{*} \lambda$. Boundaries of Stein domains are in particular of positive contact type.

2 . If the boundary $\partial M$ is of contact type and has no closed contractible characteristics, then it trivially satisfies the positive contact type property. 
3. Negative unit disc bundles satisfy the positive contact type property. In that case we have $\omega=\pi^{*} \beta+\Omega$ and $\lambda=\left(1+r^{2}\right) \theta$, where $\theta$ is the transgression 1-form (see Example 3.3). The closed characteristics are contractible in the fibers and we have, for each of them, $A_{\omega}(\gamma)=$ $\int_{\mathbb{S}^{1}} \gamma^{*} \theta=\frac{1}{2} \int_{\mathbb{S}^{1}} \gamma^{*} \lambda$.

4. I do not know any example of a symplectically aspherical manifold whose boundary is of contact type but not of positive contact type. One should note that the definition only makes sense in a symplectically aspherical manifold. If $\left\langle\omega, \pi_{2}(M)\right\rangle \neq 0$, we can always glue a sufficiently negative sphere to any filling disc $\bar{\gamma}$ so that $A_{\omega}(\gamma, \bar{\gamma})$ becomes negative.

Remark 4.27 (Computation). If $\partial E$ has the positive contact type property as defined in Section 4.3, we can compute $F H^{*}(E)$ with a cofinal family of Hamiltonians such that their 1-periodic orbits are either constant with negative action close to zero, or nonconstant with positive action [31]. Given any $a<0$, we then have

$$
F H^{*}(E)=\lim _{\leftarrow} F H_{[a, b]}^{*}(E)
$$

Under the positive contact type assumption on the fibers of $E$, the proofs of [31], based on manipulations of energy levels, apply verbatim in order to show that our fibered version of symplectic homology has the following properties.

a) ([31], Prop. 1.4) If $\mu>0$ is small enough, we have

$$
F H_{]-\infty, \mu]}^{*}(E) \simeq F H_{[-\mu, \mu]}^{*}(E) \simeq H^{*+m}(E, \partial E), \quad 2 m=\operatorname{dim} E .
$$

In particular, there is a canonical morphism $c^{*}: F H^{*}(E) \rightarrow$ $H^{*+m}(E, \partial E)$ induced by the truncation of the range of action.

b) ([31], Thm. 3.1) Any codimension 0 embedding $j: W \hookrightarrow E$ of a domain $W$ such that $\partial W$ is of positive contact type and $W$ satisfies condition (A) of [31] induces a transfer morphism $F j^{!}: F H^{*}(W) \rightarrow$ $F H^{*}(E)$ which makes the following diagram commutative

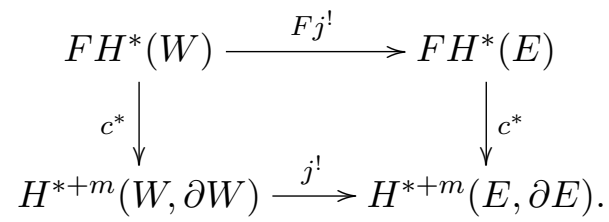

The bottom arrow is the Poincaré dual of $H_{m-*}(W) \stackrel{j_{*}}{\longrightarrow} H_{m-*}(E)$. The requirement that $\partial W$ be of positive contact type can be relaxed to the weaker assumption that $\partial W$ be the boundary of a negative symplectic fibration whose fibers satisfy the positive contact type 
condition. Moreover, if there exists no closed characteristic on $\partial W$ or if $\partial W$ is of restricted contact type in $M$, then $F j$ is defined in the symplectically aspherical case without reference to the additional condition $(\mathrm{A})$ of $[\mathbf{3 1}]$.

c) $\left([\mathbf{3 1}]\right.$, Thm. 4.1) If the map $F H^{*}(E) \longrightarrow H^{2 m}(E, \partial E)$ is not surjective, then any contact type hypersurface which bounds a domain $W$ in $M$ carries a closed characteristic. The same conclusion holds if $\partial W$ is the boundary of a negative symplectic fibration.

Remark 4.28 (Homological versus cohomological formalism). As announced in the introduction, one can build symplectic homology groups based on the same chain groups as the cohomological ones, but with dual differential

$$
\delta\langle y\rangle=\sum_{x: \operatorname{dim} \mathcal{M}(y, x)=1} \#(\mathcal{M}(y, x) / \mathbb{R})\langle x\rangle .
$$

This formula is to be compared with (4.40). The main difference between cohomology and homology is that the first involves an inverse limit, whereas the second involves a direct limit. The latter is always an exact functor and therefore the homological spectral sequence holds with integer coefficients, whereas the cohomological one holds with field coefficients. We chose to work with cohomology in order to respect the setting of [31] on which we base our applications.

\section{Pseudo-gradient vector fields}

We construct in this section a special family of almost complex structures, Hamiltonians and pseudo-gradient vector fields $\left(J_{\nu}, K_{\nu}, Y_{\nu}\right), \nu \rightarrow \infty$ which is cofinal for the previously defined order $\prec$ and which satisfies the following two properties.

A) The 1-periodic orbits of $K_{\nu}$ are located in the fibers over the critical points of a function $f: B \longrightarrow \mathbb{R}$ which is $C^{2}$-small and whose gradient flow is Morse-Smale. The gradient is computed with respect to the metric induced by a generic time-independent almost complex structure $J_{B}$.

B) Floer trajectories for the Floer complex $F C^{*}\left(J_{\nu}, Y_{\nu}\right)$ project on gradient trajectories of the Morse complex $F C^{*}\left(J_{B}, c_{\nu} f\right)$ on $B$, for some $c_{\nu}>0$.

Conditions (A) and (B) will be used in Section 7 in order to filter each Floer complex $F C^{*}\left(J_{\nu}, Y_{\nu}\right)$ by the Morse index of the projections of the 1-periodic orbits of $K_{\nu}$.

5.1. A model pseudo-gradient property. We begin by introducing horizontal distributions which are time-dependent. These play an important role 
in order to achieve transversality within the class of split almost complex structures (see Section 6). We denote by

$$
H_{0}=\operatorname{Vert}^{\perp \Omega}
$$

the horizontal distribution determined by $\Omega$, and by $H=\left(H_{t}\right), \quad t \in \mathbb{S}^{1}$ an arbitrary time-dependent horizontal distribution such that $H \equiv H_{0}$ on $\{S \geq 1\}$. Every such horizontal distribution can be described as a loop of graphs of linear maps

$$
L_{t}: H_{0} \longrightarrow \text { Vert, } \quad t \in \mathbb{S}^{1},
$$

with graph $\left(L_{t}\right) \subset H_{0} \oplus$ Vert $=T \widehat{E}$ and $L_{t}$ supported in $E$. Given a horizontal distribution $H$, we mark the horizontal lift of objects on $B$ by the symbol . In order to emphasize the horizontal distribution $H$ with respect to which we construct the lift, we shall sometimes use the superscript $H$. We point out that time-dependent horizontal distributions produce time-dependent lifts, even if the objects on $B$ are time-independent. On the other hand, the property of being vertical is independent of the choice of horizontal distribution. We denote by

$$
X=X^{\mathrm{h}}+X^{\mathrm{v}}
$$

the decomposition according to the splitting $T \widehat{E}=H_{0} \oplus$ Vert. We denote by

$$
X=X^{\text {horiz }}+X^{\text {vert }}
$$

the decomposition of a vector $X \in T \widehat{E}$ according to the splitting $T \widehat{E}=$ $H \oplus$ Vert. We have $X_{t}^{\text {horiz }}=X^{\mathrm{h}}+L_{t}\left(X^{\mathrm{h}}\right), X_{t}^{\text {vert }}=X^{\mathrm{v}}-L_{t}\left(X^{\mathrm{h}}\right)$.

In the next statement we make the following notations:

- $J_{V}$ is an almost complex structure on Vert which is compatible with $\left.\Omega\right|_{\text {Vert }}$ and which is standard on $\partial E \times[1, \infty[$;

- $J_{B}$ is an almost complex structure on $B$ compatible with $\beta$ and such that $\Omega\left(\cdot, \widetilde{J}_{B} \cdot\right)$ is positive on the horizontal distribution $H_{0}$ for $S \geq 1$;

- $f: B \longrightarrow \mathbb{R}$ is a Morse function with critical points $\left\{p_{1}, \ldots, p_{\ell}\right\}$ and $U_{i}, i \in\{1, \ldots, \ell\}$ are mutually disjoint open neighborhoods of the $p_{i}$ 's;

- $H$ is a horizontal distribution given by a loop $L=\left(L_{t}: H_{0} \longrightarrow\right.$ Vert $)$, $t \in \mathbb{S}^{1}$ supported in $E \backslash \bigcup_{i=1}^{\ell} \pi^{-1}\left(U_{i}\right)$;

- $h: \widehat{E} \longrightarrow \mathbb{R}$ is a Hamiltonian with vertical Hamiltonian vector field, linear for $S \geq 1$ with slope $\lambda_{\max }$ (typically of the form $h=h(S)$ ).

We use a superscript $\epsilon$ in order to emphasize that the Hamiltonian vector fields or the Hamiltonian action are computed with respect to $\omega_{\epsilon}=\pi^{*} \beta+\epsilon \Omega$. 
Proposition 5.1. Assume the almost complex structure $J_{B} \in J(B, \beta)$ is time-independent, and assume that the maximal slope of $h$ satisfies the condition

$$
\lambda_{\max } \notin \operatorname{Spec}(\partial E) .
$$

There exist constants $\epsilon_{0}, \delta_{0}, \rho_{0}, \alpha_{0}>0$ such that, for $\epsilon \leq \epsilon_{0}, \alpha \leq \alpha_{0}, \delta \leq \delta_{0}$ and $\|L\|_{C^{0}} \leq \rho_{0} \delta$, the following statements hold true:

- the form $\omega_{\epsilon}, 0<\epsilon \leq \epsilon_{0}$ is nondegenerate on $\widehat{E}$ and tames $J_{V} \oplus \widetilde{J}_{B}^{H}$;

- the vector field defined on the space of 1-periodic loops by

$$
\mathcal{Y}^{\epsilon}(x)=J\left(\dot{x}-X_{\epsilon h}^{\epsilon} \circ x-\widetilde{X_{\epsilon \delta f}} \circ x\right)
$$

satisfies the strong pseudo-gradient inequality

$$
d A_{\epsilon(h+\delta \widetilde{f})}^{\epsilon}(x) \cdot \mathcal{Y}^{\epsilon}(x) \geq \alpha\left\|\mathcal{Y}^{\epsilon}(x)\right\|_{\omega_{\epsilon}}^{2},
$$

with equality iff $x$ is a 1-periodic orbit of $X_{h}=X_{\epsilon h}^{\epsilon}$ in a critical fiber of $\tilde{f}$.

Remark 5.2 (The trivial case). If $X_{f} \equiv 0$ the pseudo-gradient property is clearly satisfied with $\alpha=1$ since $\mathcal{Y}^{\epsilon}=\nabla A_{\epsilon h}^{\epsilon}$.

Remark 5.3 (Comparing $\mathcal{Y}^{\epsilon}$ and $\nabla A_{\epsilon(h+\delta \widetilde{f})}^{\epsilon}$ ). Let us assume in order to simplify notation that $\epsilon=\delta=1$. Then $\mathcal{Y}(x)-\nabla A_{h+\widetilde{f}}(x)=\left(X_{\widetilde{f}}-\widetilde{X}_{f}\right) \circ x$. On the other hand, if $\left.\Omega\right|_{\partial E}$ is nondegenerate on $H_{0}$, we have $\left|X_{\tilde{f}}\right|_{\beta} \rightarrow 0$ as $S \rightarrow \infty$, whereas $\left|\widetilde{X_{f}}\right|_{\beta}$ stays constant as $S \rightarrow \infty$. The vector field $\mathcal{Y}$ is thus a "big" perturbation of $\nabla A_{h+\widetilde{f}}$ and inequality (5.1) should come as a pleasant surprise.

Proof. The statement concerning $\omega_{\epsilon}$ follows immediately from the (NEGATIVITY) assumption and from the fact that $L$ is supported in $E$, so that we are left to prove the statement concerning $\mathcal{Y}^{\epsilon}$. We note that $\mathcal{Y}^{\epsilon}(x)=J\left(\dot{x}-X_{h} \circ x-\epsilon \widetilde{X_{\delta f}} \circ x\right)$ and, in order not to burden the notation, we give the proof for $\epsilon=\epsilon_{0}=1$. The reader can easily convince himself that the proof holds verbatim for an arbitrary value $0<\epsilon<\epsilon_{0}$. The intuitive reason is that, as $\epsilon$ decreases, the factor in front of $\widetilde{f}$ is allowed to vary in the smaller interval $\left.] 0, \epsilon \delta_{0}\right]$ and the vector field $\mathcal{Y}^{\epsilon}$ gets closer to $\nabla A_{\epsilon(h+\delta \widetilde{f})}^{\epsilon}$.

Let $E=d A_{h+\widetilde{f}}(x) \cdot \mathcal{Y}(x)=d A_{h+\widetilde{f}}(x) \cdot J\left(\dot{x}-X_{h}-\widetilde{X_{f}}\right)$. We have

$$
\begin{aligned}
E & =\int \omega\left(\dot{x}, J \dot{x}-J X_{h}-J \widetilde{X_{f}}\right)-\int(d \widetilde{f}+d h) \cdot\left(J \dot{x}-J X_{h}-J \widetilde{X_{f}}\right) \\
& =\left\|\dot{x}-X_{h}\right\|_{\omega}^{2}-\int\left(\omega\left(\dot{x}, J \widetilde{X_{f}}\right)+\pi^{*} \beta\left(\widetilde{X_{f}}, J \dot{x}-J \widetilde{X_{f}}\right)-\omega\left(X_{h}, J \widetilde{X_{f}}\right)\right) \\
& =\left\|\dot{x}-X_{h}-\widetilde{X_{f}}\right\|_{\omega}^{2}+\int\left(\Omega\left(\widetilde{X_{f}}, J \dot{x}\right)-\Omega\left(\widetilde{X_{f}}, J \widetilde{X_{f}}\right)+\Omega\left(J X_{h}, \widetilde{X_{f}}\right)\right) .
\end{aligned}
$$


We distinguish three cases: either the loop $x$ is contained in $\partial E \times[1, \infty[$, either it is contained in the compact region $S \leq \Lambda$, either it intersects both $S=1$ and $S=\Lambda$. The real number $\Lambda \geq 1$ will be suitably chosen below (we will see that $\Lambda=4$ is a convenient choice).

Case 1 . We suppose that $x$ is contained in $\partial E \times\left[1, \infty\left[\right.\right.$. Because $H=H_{0}$ on $\partial E \times\left[1, \infty\left[\right.\right.$, the term $\Omega\left(J X_{h}, \widetilde{X_{f}}\right)$ vanishes and inequality (5.1) becomes

$$
\begin{aligned}
& \left(1-\alpha^{2}\right)\left\|\left(\dot{x}-X_{h}-\widetilde{X_{f}}\right)^{\mathrm{h}}\right\|_{\beta}^{2} \\
& \quad+\left(\left(1-\alpha^{2}\right)\left\|\dot{x}-X_{h}-\widetilde{X_{f}}\right\|_{\Omega}^{2}-\left\|\widetilde{X_{f}}\right\|_{\Omega}^{2}+\int \Omega\left(\widetilde{X_{f}}, J \dot{x}\right)\right) \geq 0 .
\end{aligned}
$$

It is enough to prove that the term in (5.2), which we denote by $E_{1}$, is positive. Let $x(t)=(\bar{x}(t), S(t))$. Because $X_{h}$ has no 1-periodic orbits on $\partial E \times\left[1, \infty\left[\right.\right.$ and $X_{h}$ is contained in a nondegeneracy subspace of $\Omega$, there exists $c>0$ such that any 1 -periodic loop $\bar{x}: \mathbb{S}^{1} \longrightarrow \partial E$ satisfies

$$
\int_{\mathbb{S}^{1}}\left|\dot{\bar{x}}-X_{h}\right|_{\Omega}^{2} \geq c
$$

We denote $\delta=\max _{E}\left|\widetilde{X}_{f}\right|_{\Omega}$. We let $\eta>0$ be a positive number. The condition $E_{1} \geq 0$ is invariant under homotheties and we may therefore assume that $\min _{\mathbb{S}^{1}} S(t)=1$. We have

$$
\begin{aligned}
E_{1}= & 4\left(1-\alpha^{2}\right)\left\|\sqrt{S}^{\prime}\right\|_{L^{2}}^{2}+\left(1-\alpha^{2}\right) \int_{\mathbb{S}^{1}} S(t)\left|\dot{\bar{x}}-X_{h}\right|_{\Omega}^{2}-\alpha^{2} \int_{\mathbb{S}^{1}} S(t)\left|\widetilde{X_{f}} \circ \bar{x}\right|_{\Omega}^{2} \\
& -\left(2-2 \alpha^{2}\right) \int_{\mathbb{S}^{1}} S(t)\left\langle\dot{\bar{x}}, \widetilde{X_{f}}\right\rangle_{\Omega}+\int_{\mathbb{S}^{1}} S(t) \Omega\left(\widetilde{X_{f}}, J \dot{\bar{x}}\right) \\
\geq & 4\left(1-\alpha^{2}\right)\left\|\sqrt{S}^{\prime}\right\|_{L^{2}}^{2}+\left(1-\alpha^{2}\right) \int_{\mathbb{S}^{1}} S(t)\left|\dot{\bar{x}}-X_{h}\right|_{\Omega}^{2}-\alpha^{2} \delta^{2}\|\sqrt{S}\|_{L^{2}}^{2} \\
& -\left(1-\alpha^{2}+\frac{1}{2}\|\Omega\|_{E, \Omega}\right) \int_{\mathbb{S}^{1}} S(t)\left(\eta\left|\dot{\bar{x}}-X_{h}\right|_{\Omega}^{2}+\frac{1}{\eta}\left|\widetilde{X}_{f}\right|_{\Omega}^{2}\right) \\
\geq & 4\left(1-\alpha^{2}\right)\left\|\sqrt{S}^{\prime}\right\|_{L^{2}}^{2}+\left(1-\alpha^{2}-\left(1-\alpha^{2}\right) \eta-\eta\|\Omega\|_{E, \Omega} / 2\right) \cdot c \\
& -\delta^{2}\left(\alpha^{2}+\left(1-\alpha^{2}+\frac{1}{2}\|\Omega\|_{E, \Omega}\right) / \eta\right)\|\sqrt{S}\|_{L^{2}}^{2} .
\end{aligned}
$$

This last expression is strictly positive for $\eta$ and $\delta$ small enough due to the Poincaré inequality which, for $\min _{\mathbb{S}^{1}} S(t)=1$, writes

$$
\|\sqrt{S}\|_{L^{2}} \leq 1+\left\|\sqrt{S}^{\prime}\right\|_{L^{2}} .
$$

We note that, because we do not assume $\widetilde{J}_{B}$ to be compatible with $\Omega$ on $H$, the norm $\|\Omega\|_{E, \Omega}$ of $\Omega$ as a bilinear map in the induced (possibly degenerate) metric may be arbitrarily large. One can construct explicit examples for this phenomenon. 
Remark 5.4 (Slope). The above argument crucially uses the hypothesis that the maximal slope of $h$ does not belong to $\operatorname{Spec}(\partial E)$, through inequality (5.3).

Case 2. We suppose now that $x$ intersects both regions $S<1$ and $S>\Lambda$, where $\Lambda$ is to be chosen later $\left(\Lambda=4\right.$ is a suitable choice). Let $J=\left\{t \in \mathbb{S}^{1}\right.$ : $S(x(t)) \geq 1\}$ and $J^{c}=\mathbb{S}^{1} \backslash J$. We can assume without loss of generality that $x$ has transverse intersection with $\partial E$, in which case $J$ is a finite union of intervals $J_{k}, k \in\{1, \ldots, N\}$ and $\left.S \circ x\right|_{\partial J_{k}} \equiv 1$. Let $\delta^{2}=\max _{B}\left|\beta\left(X_{f}, J_{B} X_{f}\right)\right|$.

We must prove that $E^{\prime} \geq 0$, where

$$
\begin{aligned}
E^{\prime}= & \left(1-\alpha^{2}\right)\left\|\dot{x}-X_{h}-\widetilde{X_{f}}\right\|_{\omega}^{2}+\int \Omega\left(\widetilde{X_{f}}, J \dot{x}-J X_{h}-J \widetilde{X_{f}}\right) \\
= & \left(1-\alpha^{2}\right) \int_{J^{c}}\left|\dot{x}-X_{h}-\widetilde{X_{f}}\right|_{\omega}^{2}+\int_{J^{c}} \Omega\left(\widetilde{X_{f}}, J \dot{x}-J X_{h}-J \widetilde{X_{f}}\right) \\
& +\left(1-\alpha^{2}\right) \int_{J}\left|\dot{x}-X_{h}-\widetilde{X_{f}}\right|_{\omega}^{2}+\int_{J} \Omega\left(\widetilde{X_{f}}, J \dot{x}-J X_{h}-J \widetilde{X_{f}}\right) .
\end{aligned}
$$

For $\epsilon_{0}$ small enough, we have $\|\Omega\|_{\beta} \leq 1$ and $|\cdot|_{\omega} \geq \frac{1}{2}|\cdot|_{\beta}$ on $E$, hence $\left|\Omega\left(\widetilde{X_{f}}, J \dot{x}-J X_{h}-J \widetilde{X_{f}}\right)\right| \leq \delta\left|\dot{x}-X_{h}-\widetilde{X_{f}}\right|_{\beta} \leq 2 \delta\left|\dot{x}-X_{h}-\widetilde{X_{f}}\right|_{\omega}$. We therefore obtain

$$
\begin{aligned}
& \left(1-\alpha^{2}\right) \int_{J^{c}}\left|\dot{x}-X_{h}-\widetilde{X}_{f}\right|_{\omega}^{2}+\int_{J^{c}} \Omega\left(\widetilde{X_{f}}, J \dot{x}-J X_{h}-J \widetilde{X_{f}}\right) \\
& \quad \geq \int_{J^{c}}\left(\sqrt{1-\alpha^{2}}\left|\dot{x}-X_{h}-\widetilde{X_{f}}\right|_{\omega}-\delta / \sqrt{1-\alpha^{2}}\right)^{2}-\int_{J^{c}} \delta^{2} /\left(1-\alpha^{2}\right) \\
& \quad \geq-\delta^{2} /\left(1-\alpha^{2}\right) .
\end{aligned}
$$

On the other hand, we have $x(t) \in \partial E \times\left[1, \infty\left[\right.\right.$ for $t \in J$ and $H=H_{0}$ on $\partial E \times[1, \infty[$. If $\eta$ is a small enough positive real, we get

$$
\begin{aligned}
(1- & \left.\alpha^{2}\right) \int_{J}\left|\dot{x}-X_{h}-\widetilde{X}_{f}\right|_{\omega}^{2}+\int_{J} \Omega\left(\widetilde{X_{f}}, J \dot{x}-J X_{h}-J \widetilde{X_{f}}\right) \\
\geq & 4\left(1-\alpha^{2}\right)\left\|\bar{S}^{\prime}\right\|_{L^{2}(J)}^{2}+\left(1-\alpha^{2}-\eta\|\Omega\|_{E, \Omega} / 2\right) \int_{J} S(t) \mid \dot{\bar{x}}-X_{h} \\
& \quad-\left.\widetilde{X}_{f}\right|_{\Omega} ^{2}-\frac{1}{2 \eta}\|\Omega\|_{E, \Omega} \cdot \int_{J} S(t)\left|\widetilde{X_{f}} \circ \bar{x}\right|_{\Omega}^{2} \\
\geq & 4\left(1-\alpha^{2}\right)\left\|\sqrt{S}^{\prime}\right\|_{L^{2}(J)}^{2}-\frac{\delta^{2}}{2 \eta}\|\Omega\|_{E, \Omega} \cdot\|\sqrt{S}\|_{L^{2}(J)}^{2} .
\end{aligned}
$$

We denote $A=4\left(1-\alpha^{2}\right), B=1 /\left(1-\alpha^{2}\right), C=\|\Omega\|_{E, \Omega} / 2 \eta$ and we have obtained

$$
E^{\prime} \geq A\left\|\sqrt{S}^{\prime}\right\|_{L^{2}(J)}-B \delta^{2}-C \delta^{2}\|\sqrt{S}\|_{L^{2}(J)}^{2}
$$


The Poincaré inequality for a positive function $f$ defined on an interval $I$ of length $a$ gives

$$
\|f\|_{L^{2}(I)}^{2} \leq a\left(m+\left\|f^{\prime}\right\|_{L^{1}(I)}\right)^{2} \leq a\left(m+\sqrt{a}\left\|f^{\prime}\right\|_{L^{2}(I)}\right)^{2},
$$

where $m=\min _{I} f \geq 0$. If $a_{k}$ denotes the length of $J_{k}$, with $\sum_{k} a_{k}<1$, we obtain

$$
\begin{aligned}
\|\sqrt{S}\|_{L^{2}(J)}^{2} & \leq \sum_{k} a_{k}\left(1+\sqrt{a_{k}}\left\|\sqrt{S}^{\prime}\right\|_{L^{2}\left(J_{k}\right)}\right)^{2} \\
& \leq 1+2\left\|\sqrt{S}^{\prime}\right\|_{L^{2}(J)}+\left\|\sqrt{S}^{\prime}\right\|_{L^{2}(J)}^{2}=\left(1+\left\|\sqrt{S}^{\prime}\right\|_{L^{2}(J)}\right)^{2} .
\end{aligned}
$$

At this point we exploit the hypothesis on $x$ in order to produce a lower bound on $\left\|\sqrt{S}^{\prime}\right\|_{L^{2}(J)}$. Let $I=\left[t_{0}, t_{1}\right] \subset J$ be an interval such that $\left.S \circ x\right|_{I} \geq 1, S\left(x\left(t_{0}\right)\right)=1$ and $S\left(x\left(t_{1}\right)\right)=M$, with $M=\max S \circ x>\Lambda$. We have

$$
\left\|\sqrt{S}^{\prime}\right\|_{L^{2}(J)} \geq\left\|\sqrt{S}^{\prime}\right\|_{L^{1}(J)} \geq\left\|\sqrt{S}^{\prime}\right\|_{L^{1}(I)} \geq \sqrt{M}-1>\sqrt{\Lambda}-1 .
$$

We claim that (5.4) is strictly positive if we choose $\Lambda=4, \alpha \leq \frac{1}{2}$ and $\delta$ such that $\delta^{2} B \leq 1$ and $\delta^{2} C \leq \frac{1}{4}$. Indeed, we obtain $\|\sqrt{S}\|_{L^{2}(J)}^{2}<4\left\|\sqrt{S}^{\prime}\right\|_{L^{2}(J)}^{2}$ and the expression in (5.4) is bigger than $\left\|\sqrt{S}^{\prime}\right\|_{L^{2}(J)}^{2}$, hence bigger than 1 .

Case 3. We suppose $x$ is contained in the region $\{S \leq \Lambda\}$. We choose $\epsilon_{0}$ small enough so that $\left.|\cdot|\right|_{\omega} ^{2} \geq \frac{2}{3}|\cdot|_{\beta}^{2}$ on $E$ and $\left\|\left.\Omega\right|_{H_{0}}\right\|_{\{S \leq \Lambda\}, \beta} \leq 1 / 2$. We write

$$
\begin{aligned}
E^{\prime}= & \left(1-\alpha^{2}\right)\left\|\dot{x}-X_{h}\right\|_{\omega}^{2}-2\left(1-\alpha^{2}\right)\left\langle\dot{x}-X_{h}, \widetilde{X_{f}}\right\rangle_{\omega}+\int \Omega\left(\widetilde{X_{f}}, J \dot{x}\right) \\
& +\left\|{\widetilde{X_{f}}}^{\mathrm{h}}\right\|_{\beta}^{2}-\alpha^{2}\left\|\widetilde{X_{f}}\right\|_{\omega}^{2}+\int \Omega\left(J X_{h}, \widetilde{X_{f}}\right) .
\end{aligned}
$$

The choice of a small enough constant $\rho=\rho(f, h)$ ensures $\left|\Omega\left(J X_{h}, \widetilde{X_{f}}\right)\right| \leq$ $\alpha^{2}\left|\widetilde{X}_{f}^{\mathrm{h}}\right|_{\beta}^{2}$ and $\left|\Omega\left(X_{h}, \widetilde{X_{f}}\right)\right| \leq \alpha^{2}\left|\widetilde{X}_{f}^{\mathrm{h}}\right|_{\beta}^{2}$ pointwise. Let us argue for $\Omega\left(J X_{h}, \widetilde{X_{f}}\right)$. The inequality is clearly true on $\bigcup_{i=1}^{k} \pi^{-1}\left(U_{i}\right) \bigcup \partial E \times[1, \infty[$, where $\Omega\left(J X_{h}, \widetilde{X_{f}}\right)=0$. On $E \backslash \bigcup_{i=1}^{k} \pi^{-1}\left(U_{i}\right)$ there exists $\eta>0$ such that $\left|\widetilde{X}_{f}\right|_{\beta}^{2} \geq \eta$. On the other hand,

$$
\left|\Omega\left(J X_{h}, \widetilde{X_{f}}\right)\right|=\left|\Omega\left(J X_{h}, L\left({\widetilde{X_{f}}}^{\mathrm{h}}\right)\right)\right| \leq\|\Omega\|_{\infty, E}\left\|X_{h}\right\|_{\infty, E}\|L\|_{\infty, E}\left|{\widetilde{X_{f}}}^{\mathrm{h}}\right|_{\beta},
$$

where $\|\cdot\|_{\infty, E}=\max _{E}|\cdot|_{\beta}$. By choosing $\rho<\alpha^{2} \eta /\|\Omega\|_{\infty, E}\left\|X_{h}\right\|_{\infty, E}$, we get $\left|\Omega\left(J X_{h}, \widetilde{X_{f}}\right)\right| \leq \alpha^{2}\left|\widetilde{X_{f}}\right|_{\beta}^{2}$. Because $\rho$ depends on $\eta$, the actual constant that we get in the statement of the Proposition is of the type $\rho(f, h, \epsilon) \delta$. The same argument applies to $\Omega\left(X_{h}, \widetilde{X_{f}}\right)$. 
By further diminishing $\rho$ we can achieve $\left|\widetilde{X}_{f}\right|_{\beta}^{2} \leq 2\left|\widetilde{X}_{f}^{\mathrm{h}}\right|_{\beta}^{2}$. Indeed, we have

$\left|\widetilde{X_{f}}\right|_{\beta}^{2}=\left|\widetilde{X}_{f}^{\mathrm{h}}\right|_{\beta}^{2}+\left|L\left({\widetilde{X_{f}}}^{\mathrm{h}}\right)\right|_{\Omega}^{2} \leq\left(1+\|\Omega\|_{\{S \leq \Lambda\}, \beta}\|L\|_{\infty, E}^{2}\right)\left|{\widetilde{X_{f}}}^{\mathrm{h}}\right|_{\beta}^{2} \leq 2\left|{\widetilde{X_{f}}}_{f}^{\mathrm{h}}\right|_{\beta}^{2}$.

We infer that, for $\epsilon_{0}$ and $\rho$ small enough, the expression in (5.6), which we denote by $E_{2}^{\prime}$, satisfies

$$
E_{2}^{\prime} \geq\left(1-4 \alpha^{2}\right)\left\|\widetilde{X}_{f}^{\mathrm{h}}\right\|_{\beta}^{2} .
$$

We denote the expression in (5.5) by $E_{1}^{\prime}$. It satisfies

$$
\begin{aligned}
E_{1}^{\prime}= & \left(1-\alpha^{2}\right)\left\|\left(\dot{x}-X_{h}\right)^{\mathrm{v}}\right\|_{\Omega}^{2}-\left(1-2 \alpha^{2}\right)\left\langle\left(\dot{x}-X_{h}\right)^{\mathrm{v}}, \widetilde{X}_{f}^{\mathrm{v}}\right\rangle_{\Omega} \\
& +\left(1-\alpha^{2}\right)\left\|\dot{x}^{\mathrm{h}}\right\|_{\omega}^{2}-2\left(1-\alpha^{2}\right)\left\langle\dot{x}^{\mathrm{h}}, \widetilde{X}_{f}^{\mathrm{h}}\right\rangle_{\omega}+\int \Omega\left(\widetilde{X}_{f}^{\mathrm{h}}, J \dot{x}^{\mathrm{h}}\right) \\
= & \left(1-\alpha^{2}\right)\left\|\left(\dot{x}-X_{h}\right)^{\mathrm{v}}-\frac{1-2 \alpha^{2}}{2\left(1-\alpha^{2}\right)} \widetilde{X}_{f}^{\mathrm{v}}\right\|_{\Omega}^{2}-\frac{\left(1-2 \alpha^{2}\right)^{2}}{4\left(1-\alpha^{2}\right)}\left\|\widetilde{X}_{f}^{\mathrm{v}}\right\|_{\Omega}^{2} \\
& +\frac{1-\alpha^{2}}{2}\left\|\dot{x}^{\mathrm{h}}\right\|_{\omega}^{2}-2\left(1-\alpha^{2}\right)\left\langle\dot{x}^{\mathrm{h}}, \widetilde{X}_{f}^{\mathrm{h}}\right\rangle_{\beta} \\
& +\frac{1-\alpha^{2}}{2}\left\|\dot{x}^{\mathrm{h}}\right\|_{\omega}^{2}-\left(1-\alpha^{2}\right) \int \Omega\left(\dot{x}^{\mathrm{h}}, J \widetilde{X}_{f}^{\mathrm{h}}\right)+\alpha^{2} \int \Omega\left(\widetilde{X}_{f}^{\mathrm{h}}, J \dot{x}^{\mathrm{h}}\right),
\end{aligned}
$$

hence

$$
\begin{aligned}
E_{1}^{\prime} \geq & -\frac{\rho^{2}}{4\left(1-\alpha^{2}\right)}\left\|\widetilde{X}_{f}^{\mathrm{h}}\right\|_{\beta}^{2}+\frac{1-\alpha^{2}}{3}\left\|\dot{x}^{\mathrm{h}}\right\|_{\beta}^{2}-2\left(1-\alpha^{2}\right)\left\langle\dot{x}^{\mathrm{h}}, \widetilde{X}_{f}^{\mathrm{h}}\right\rangle_{\beta} \\
& +\frac{1-\alpha^{2}}{3}\left\|\dot{x}^{\mathrm{h}}\right\|_{\beta}^{2}-\frac{1}{2} \int\left|\dot{x}^{\mathrm{h}}\right|_{\beta}\left|\widetilde{X}_{f}^{\mathrm{h}}\right|_{\beta} \\
\geq & -\left(\frac{1}{4}+\frac{\rho^{2}}{4}\right)\left\|{\widetilde{X_{f}}}^{\mathrm{h}}\right\|_{\beta}^{2}+\left(\frac{1-\alpha^{2}}{3}-\frac{1}{4}\right)\left\|\dot{x}^{\mathrm{h}}\right\|_{\beta}^{2} \geq \\
& -\left(\frac{1}{4}+\frac{\rho^{2}}{4}\right)\left\|\widetilde{X}_{f}^{\mathrm{h}}\right\|_{\beta}^{2} .
\end{aligned}
$$

We have used for the first inequality that $\left|\widetilde{X}_{f}^{\mathrm{v}}\right|_{\Omega}^{2} \leq \rho^{2}\left|\widetilde{X}_{f}^{\mathrm{h}}\right|_{\beta}^{2},\left\|\dot{x}^{\mathrm{h}}\right\|_{\omega}^{2}$ $\geq \frac{2}{3}\left\|\dot{x}^{\mathrm{h}}\right\|_{\beta}^{2}$ and $\|\Omega\|_{\{S \leq \Lambda\}, \beta} \leq \frac{1}{2}$. We have used for the second inequality the fact that $\left(1-\alpha^{2}\right) / 3\left\|\dot{x}^{\mathrm{h}}\right\|_{\beta}^{2}-2\left(1-\alpha^{2}\right)\left\langle\dot{x}^{\mathrm{h}}, \widetilde{X}_{f}^{\mathrm{h}}\right\rangle_{\beta} \geq 0$ if $\delta$ is small enough, by Lemma 5.5 below applied with $(W, g)=\left(B, g_{B}\right), g_{B}(\cdot, \cdot)=$ $\beta\left(\cdot, J_{B} \cdot\right)$ and $\eta=1 / 12$. We have also used the inequality $\left|\dot{x}^{\mathrm{h}}\right|_{\beta}\left|{\widetilde{X_{f}}}^{\mathrm{h}}\right|_{\beta} \leq$ $\frac{1}{2}\left(\left|\dot{x}^{\mathrm{h}}\right|_{\beta}^{2}+\left|\widetilde{X}_{f}^{\mathrm{h}}\right|_{\beta}^{2}\right)$. Finally, the last inequality holds if $\left(1-\alpha^{2}\right) / 3-1 / 4 \geq 0$. As a conclusion, we obtain

$$
E^{\prime} \geq\left(\frac{3}{4}-4 \alpha^{2}-\frac{\rho^{2}}{4}\right)\left\|\widetilde{X}_{f}^{\mathrm{h}}\right\|_{\beta}^{2}
$$


hence $E^{\prime} \geq 0$ if $\alpha$ and $\rho$ are small enough. The equality case is readily characterized from this last inequality.

Lemma 5.5. Let $(W, g)$ be a compact Riemannian manifold. If $\eta>0$ is small enough, any time-independent $C^{1}$-vector field $X$ on $W$ with $\|X\|_{C^{1}}<$ $\eta$ satisfies

$$
\|\dot{x}\|_{L^{2}}^{2} \geq \frac{1}{2 \eta}\langle\dot{x}, X \circ x\rangle_{L^{2}}
$$

for any 1-periodic loop $x$ of class $C^{1}$. Equality is achieved if and only if $x$ is a constant loop.

Proof. We embed isometrically $(W, g)$ into Euclidean space and, for $\eta$ small enough, we can extend $X$ to a vector field supported near the image of $W$ and whose derivative is pointwise bounded by $2 \eta$. We can thus assume without loss of generality that $(W, g)$ is Euclidean space with the standard metric, and $X$ is a compactly supported vector field such that $\|d X\|_{\infty} \leq 2 \eta$. Since $\langle\dot{x}$, ct. $\rangle=0$ and since we only impose a hypothesis on $\|d X\|_{\infty}$, we can further assume that $X(0)=0$ and $x(0)=0$. By the Poincaré inequality we obtain

$$
\left|\langle\dot{x}, X \circ x\rangle_{L^{2}}\right| \leq\|\dot{x}\|_{L^{2}}\|X \circ x\|_{L^{2}} \leq\|d X\|_{\infty} \cdot\|\dot{x}\|_{L^{2}}^{2} \leq 2 \eta\|\dot{x}\|_{L^{2}}^{2} .
$$

The equality case is readily characterized.

Remark 5.6 (Small orbits). Inequality (5.7) implies in particular $\| \dot{x}-X \circ$ $x\left\|_{L^{2}} \geq\right\| X \circ x \|_{L^{2}}$. It should therefore be seen as a quantitative expression of the well-known fact that a vector field which is small-enough in $C^{1}$-norm has no nonconstant 1-periodic orbits. In particular, inequality (5.7) does not hold if $X$ is time-dependent.

Remark 5.7 (Asphericity). Case 3 in the proof of Proposition 5.1 is the one crucially involving the fact that $J_{B}$ does not depend on time through the use of Lemma 5.5. This is one important reason for requiring the base to be symplectically aspherical in the construction of the spectral sequence: it is the only case where transversality for Floer's equation can be achieved within the class of time-independent almost complex structures.

5.2. Definition of the geometric Hamiltonians. We construct now the Hamiltonians $K_{\nu}$ announced in the introduction of the present section. We recall that the vertical coordinate $S$ was defined only in a neighborhood of $\partial E$ as $S \in[1-\delta, 1]$. We abandon in this section the notation that we have used in Section 4.1 and do not consider anymore the function $S$ as being extended over $E$.

We first define Hamiltonians $H_{\nu}: \widehat{E} \longrightarrow \mathbb{R}, \nu \in \mathbb{N}$ by the formula

$$
H_{\nu}=h_{\nu}+c_{\nu} \tilde{f}
$$


Here $f: B \longrightarrow \mathbb{R}$ is a Morse function on $B$, its lift to $\widehat{E}$ is $\widetilde{f}=f \circ \pi$, while $\left(c_{\nu}\right)_{\nu}$ is a decreasing sequence of strictly positive real numbers converging to zero, to be chosen below.

We define the function $h_{\nu}$ as follows. We fix a strictly increasing sequence $\lambda_{\nu}$ of positive real numbers which do not belong to the period spectrum of $\partial E$ and satisfy $\lambda_{\nu} \longrightarrow \infty, \nu \longrightarrow \infty$. We choose $h_{\nu}$ to be a smooth function which is constant on $\widehat{E} \backslash \partial E \times\left[1-\frac{\delta}{2}, \infty\right.$ [ and depends only on $S$ on $\partial E \times[1-$ $\frac{\delta}{2}, \infty\left[\right.$. We shall use the notation $h_{\nu}(S)$ both for the function $h_{\nu}$ and for the corresponding function on $\left[1-\frac{\delta}{2}, \infty\left[\right.\right.$. We denote by $T_{0}$ the smallest element of $\operatorname{Spec}(\partial E)$ and use the convention $T_{0}=\infty$ if $\operatorname{Spec}(\partial E)=\emptyset$. We impose the following conditions on $h_{\nu}$ :

- $h_{\nu}(S)$ is strictly convex on $] 1-\frac{\delta}{2}, 1\left[\right.$ and $h_{\nu}^{\prime}\left(1-\frac{\delta}{4}\right)<T_{0}$;

- $h_{\nu}^{\prime}(S)=\lambda_{\nu}$ for $S \geq 1-\frac{\delta}{8}$

- $h_{\nu}<0$ on $E$.

We note that the smoothness assumption on $h_{\nu}$ implies that $h_{\nu}^{\prime}$ vanishes at infinite order at $S=1-\frac{\delta}{2}$. We moreover require that

- for every $\nu \in \mathbb{N}$ we have $h_{\nu}+c_{\nu} \widetilde{f}<0$ on $E$;

- for $\nu<\nu^{\prime}$ we have $h_{\nu}<h_{\nu^{\prime}}$, and therefore $H_{\nu}<H_{\nu^{\prime}}$ for $c_{\nu}$ small enough.

Because $X_{h_{\nu}}$ is vertical, Proposition 5.1 implies that the vector field $\left(J_{V} \oplus \widetilde{J}_{B}\right)\left(\dot{x}-X_{h_{\nu}} \circ x-\epsilon c_{\nu} \widetilde{X_{f}} \circ x\right), x \in \Lambda_{0} \widehat{E}$ is a strong negative pseudogradient for the action functional $A_{\epsilon H_{\nu}}^{\epsilon}$ if $\lambda_{\max } \notin \operatorname{Spec}(\partial E)$ and $c_{\nu}$ is small enough. The lift $\widetilde{X_{f}}$ is considered here with respect to a horizontal distribution which is close enough to $H_{0}$.

The 1-periodic orbits of $H_{\nu}$ are all degenerate and fall in two classes:

1) constants in the critical fibers of $\left.\widetilde{f}\right|_{\widehat{E} \backslash \partial E \times] 1-\frac{\delta}{2}, \infty[}$;

2) nontrivial orbits in the critical fibers of $\widetilde{f}$, appearing in the region $\left\{1-\frac{\delta}{4}<S<1-\frac{\delta}{8}\right\}$ and corresponding to closed characteristics with period smaller than $\lambda_{\nu}$. The characteristics are understood to be parametrized by $X_{\text {Reeb }}$. In the best of the situations, they are transversally nondegenerate.

We construct now Hamiltonians $K_{\nu}$ with nondegenerate 1-periodic orbits by perturbing $H_{\nu}$. We need two kinds of perturbations, corresponding to the above two types of orbits:

(A) a time-independent perturbation localized in a neighborhood of the critical fibers of $\left.\widetilde{f}\right|_{\widehat{E} \backslash \partial E \times] 1-\frac{\delta}{2}, \infty[}$

(B) a time-dependent perturbation localized in a neighborhood of the nonconstant 1-periodic orbits. 
(A) Let $\left\{p_{1}, \ldots, p_{\ell}\right\}$ be the critical points of the Morse function $f: B \longrightarrow$ $\mathbb{R}$. We denote $F_{i}=F_{p_{i}}$ and $S_{i}=\left.S\right|_{F_{i}}$. We choose mutually disjoint open sets $U_{i} \ni p_{i}$ admitting trivializations $\Psi_{i}: \pi^{-1}\left(U_{i}\right) \stackrel{\sim}{\longrightarrow} U_{i} \times F_{i}$ such that $S_{i} \circ \operatorname{pr}_{2} \circ \Psi_{i}=\left.S\right|_{\pi^{-1}\left(U_{i}\right)}$ and $\Psi_{*} H_{y}=\bar{H}_{y}$ for all $y \in F_{i}$, where $\bar{H}$ is the trivial horizontal distribution on $U_{i} \times F_{i}$. Such a trivialization can be constructed by parallel transport along the radii of a geodesic ball centered at $p_{i}$. This type of trivialization is even a symplectic diffeomorphism in the fibers, but we shall not use this fact.

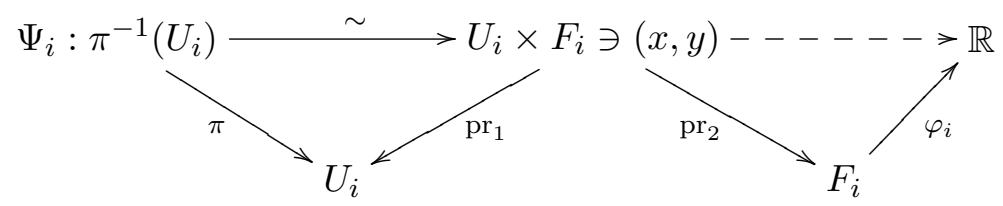

We choose now functions $\varphi_{i}: F_{i} \longrightarrow \mathbb{R}$ subject to the following conditions:

- $\varphi_{i}=0$ for $1-\frac{\delta}{4} \leq S \leq 1$;

- $\varphi_{i}=\varphi_{i}(S)$ for $1-\frac{\delta}{2} \leq S<1-\frac{\delta}{4}$, a strictly concave function satisfying $\left|\varphi_{i}^{\prime}\left(1-\frac{\delta}{2}\right)\right|<T_{0}$

- $\varphi_{i}$ is a Morse function on $E \backslash \partial E \times\left[1-\frac{\delta}{4}, 1\right]$.

We denote by $f_{i}$ the composition $\varphi_{i} \circ \mathrm{pr}_{2} \circ \Psi_{i}: \pi^{-1}\left(U_{i}\right) \longrightarrow \mathbb{R}$. We choose relatively compact open subsets $V_{i} \Subset U_{i}, p_{i} \in V_{i}$ and smooth compactly supported cut-off functions $\rho_{i}: U_{i} \longrightarrow \mathbb{R}$ such that $\left.\rho_{i}\right|_{V_{i}}=1$ and $0 \leq \rho_{i} \leq 1$.

We now define the first perturbation $\widetilde{K}_{\nu}$ of $H_{\nu}$ to be

$$
\widetilde{K}_{\nu}=H_{\nu}+c_{\nu}^{\prime} \sum_{p_{i} \in \operatorname{Crit}(f)} \tilde{\rho}_{i} \cdot f_{i}
$$

Here $c_{\nu}^{\prime}$ is a decreasing sequence of strictly positive real numbers, with $c_{\nu}^{\prime}$ small enough such that the only critical points of $\widetilde{K}_{\nu}$ inside $E$ are the critical points of $f_{i}$ in $F_{i}$.

(B) The Hamiltonian $\widetilde{K}_{\nu}$ has nontrivial 1-periodic orbits in the critical fibers $F_{i}$ in the region $1-\frac{\delta}{4}<S<1$. For any $\delta>0$, there exists a time-dependent Hamiltonian $\chi_{\nu, i}^{\delta}: \mathbb{S}^{1} \times F_{i} \longrightarrow \mathbb{R}$, with $\left\|\chi_{\nu, i}^{\delta}\right\|_{C^{2}} \leq \delta$ and supported in an arbitrarily small neighborhood of the nontrivial 1-periodic orbits of $\left.\widetilde{K}_{\nu}\right|_{F_{i}}$, such that the 1-periodic orbits of $\left.\widetilde{K}_{\nu}\right|_{F_{i}}+\chi_{\nu, i}^{\delta}$ are nondegenerate. We denote $G_{\nu, i}^{\delta}=\chi_{\nu, i}^{\delta} \circ\left(\mathrm{id} \times\left(\operatorname{pr}_{2} \circ \Psi_{i}\right)\right): \mathbb{S}^{1} \times \pi^{-1}\left(U_{i}\right) \longrightarrow \mathbb{R}$. We define

$$
K_{\nu}=\widetilde{K}_{\nu}+c_{\nu}^{\prime} \sum_{p_{i} \in \operatorname{Crit}(f)} \widetilde{\rho}_{i} \cdot G_{\nu, i}^{\delta} .
$$

The property $\Psi_{*} H_{y}=\bar{H}_{y}, y \in F_{i}$ ensures that $X_{G_{\nu, i}^{\delta}}$ and $X_{f_{i}}$ are vertical along the fibers $F_{i}$. We infer the existence of a constant $m>0$ depending 
on all the choices made before such that, for a given $\nu$, we have

$$
\begin{aligned}
\left|X_{G_{\nu, i}^{\delta}}^{\mathrm{h}}\right|_{\beta} & \leq m\left|\widetilde{X}_{f}^{\mathrm{h}}\right|_{\beta}, \\
\left|X_{f_{i}}^{\mathrm{h}}\right|_{\beta} & \leq m\left|{\widetilde{X_{f}}}^{\mathrm{h}}\right|_{\beta} .
\end{aligned}
$$

Moreover, by multiplying $G_{\nu, i}^{\delta}$ and $f_{i}$ by sufficiently small positive reals, we can achieve that the above two inequalities hold for all $\nu$ and $i$ with a uniform constant $m>0$, which can moreover be chosen arbitrarily small.

5.3. Geometric pseudo-gradient vector fields. The objects that we consider in the next statement are those of Proposition 5.1, namely an almost complex structure $J=J_{V} \oplus \widetilde{J}_{B}$, a Morse function $f: B \rightarrow \mathbb{R}$, a horizontal distribution $H$ given by a loop $L=\left(L_{t}: H_{0} \rightarrow\right.$ Vert $)$ and a Hamiltonian $h$ with vertical Hamiltonian vector field. We consider in addition a time-dependent pertubation $G$ supported in $E \cap \bigcup_{i=1}^{\ell} \pi^{-1}\left(U_{i}\right)$ satisfying the inequality

$$
\left|X_{G}^{\mathrm{h}}\right|_{\beta} \leq m\left|\widetilde{X}_{f}^{\mathrm{h}}\right|_{\beta}
$$

for some $m>0$.

Proposition 5.8. Assume the almost complex structure $J_{B} \in \mathcal{J}(B, \beta)$ is time-independent, and assume that the maximal slope of $h$ satisfies the condition

$$
\lambda_{\max } \notin \operatorname{Spec}(\partial E) .
$$

There exist constants $\epsilon_{0}, \delta_{0}, \rho_{0}, \alpha_{0}, m_{0}>0$ such that, for $\epsilon \leq \epsilon_{0}, \alpha \leq \alpha_{0}$, $\delta \leq \delta_{0},\|L\|_{C^{0}} \leq \rho_{0} \delta$ and $m \leq m_{0}$, the vector field defined on the space of 1-periodic loops by

$$
\mathcal{Y}^{\epsilon}(x)=J\left(\dot{x}-X_{\epsilon h}^{\epsilon} \circ x-\widetilde{X_{\epsilon \delta f}} \circ x-\left(X_{\epsilon G}^{\epsilon}\right)^{\mathrm{v}} \circ x\right)
$$

satisfies the strong pseudo-gradient inequality

$$
d A_{\epsilon(h+\delta \tilde{f}+G)}^{\epsilon}(x) \cdot \mathcal{Y}^{\epsilon}(x) \geq \alpha\left\|\mathcal{Y}^{\epsilon}(x)\right\|_{\omega_{\epsilon}}^{2} .
$$

Equality holds iff $x$ is a periodic orbit of $X_{h+G}=X_{\epsilon(h+G)}^{\epsilon}$ in a critical fiber of $\tilde{f}$.

Proof. We follow the proof of Proposition 5.1 and we again assume without loss of generality that $\epsilon=1$ in order not to burden the notation. Because $G$ is supported in $E$, the proof of Case 1 remains unchanged. The specific feature of Case 2 is the Poincaré inequality for loops and this remains unchanged as well, although some new estimates are needed in the preliminary computations. These estimates appear also in the proof of Case 3, and 
we give full details only for this last case. We denote $E=d A_{h+\widetilde{f}+G} \cdot \mathcal{Y}(x)$ and assume that $x$ is contained in $\{S \leq \Lambda\}$. We have

$$
\begin{aligned}
E= & \int \omega\left(\dot{x}, J\left(\dot{x}-X_{h}-\widetilde{X_{f}}-X_{G}^{\mathrm{v}}\right)\right)-d(h+\widetilde{f}+G) \cdot J\left(\dot{x}-X_{h}-\widetilde{X_{f}}-X_{G}^{\mathrm{v}}\right) \\
= & \left\|\dot{x}-X_{h}-\widetilde{X_{f}}-X_{G}^{\mathrm{v}}\right\|_{\omega}^{2}-\int \Omega\left(\widetilde{X_{f}}, J \widetilde{X_{f}}\right)+\int \Omega\left(\widetilde{X_{f}}, J \dot{x}-J X_{h}\right) \\
& -\int \omega\left(X_{G}^{\mathrm{h}}, J \dot{x}-J \widetilde{X_{f}}\right) .
\end{aligned}
$$

Inequality (5.11) is equivalent to

$$
\begin{aligned}
& \left(1-\alpha^{2}\right)\left\|\dot{x}-X_{h}-\widetilde{X_{f}}-X_{G}^{\mathrm{v}}\right\|_{\omega}^{2}-\int \Omega\left(\widetilde{X_{f}}, J \widetilde{X_{f}}\right) \\
& \quad+\int \Omega\left(\widetilde{X_{f}}, J \dot{x}-J X_{h}\right)-\int \omega\left(X_{G}^{\mathrm{h}}, J \dot{x}-J \widetilde{X_{f}}\right) \geq 0 .
\end{aligned}
$$

The left hand side of the above inequality can be written as

$$
\begin{aligned}
E^{\prime}= & \left(1-\alpha^{2}\right)\left\|\dot{x}-X_{h}-X_{G}^{\mathrm{v}}\right\|_{\omega}^{2}-2\left(1-\alpha^{2}\right)\left\langle\dot{x}-X_{h}-X_{G}^{\mathrm{v}}, \widetilde{X_{f}}\right\rangle_{\omega} \\
& +\int \Omega\left(\widetilde{X_{f}}, J \dot{x}\right)-\int \omega\left(X_{G}^{\mathrm{h}}, J \dot{x}\right) \\
& +\left(1-\alpha^{2}\right)\left\|\widetilde{X}_{f}^{\mathrm{h}}\right\|_{\beta}^{2}-\alpha^{2} \int \Omega\left(\widetilde{X_{f}}, J \widetilde{X_{f}}\right) \\
& -\int \Omega\left(\widetilde{X_{f}}, J X_{h}\right)+\int \omega\left(X_{G}^{\mathrm{h}}, J \widetilde{X_{f}}\right)
\end{aligned}
$$

For $\epsilon_{0}$ and $\rho$ small enough, the expression $E^{\prime \prime}$ obtained by summing up (5.14) and (5.15) satisfies

$$
E^{\prime \prime} \geq\left(1-3 \alpha^{2}-2 m\right)\left\|\widetilde{X}_{f}^{\mathrm{h}}\right\|_{\beta}^{2}
$$

Again for $\epsilon_{0}$ and $\rho$ small enough, we break the expression obtained by summing up (5.12) and (5.13) as a sum $E_{1}^{\prime \prime}+E_{2}^{\prime \prime}$ as follows.

$$
\begin{aligned}
E_{1}^{\prime \prime}= & \left(1-\alpha^{2}\right)\left\|\dot{x}^{\mathrm{v}}-X_{h}-X_{G}^{\mathrm{v}}\right\|_{\Omega}^{2} \\
& -\left(2-2 \alpha^{2}\right)\left\langle\dot{x}^{\mathrm{v}}-X_{h}-X_{G}^{\mathrm{v}}, \widetilde{X}_{f}^{\mathrm{v}}\right\rangle_{\Omega}+\int \Omega\left({\widetilde{X_{f}}}^{\mathrm{v}}, J \dot{x}^{\mathrm{v}}\right)
\end{aligned}
$$




$$
\begin{aligned}
= & \left(1-\alpha^{2}\right)\left\|\dot{x}^{\mathrm{v}}-X_{h}-X_{G}^{\mathrm{v}}-\frac{1-2 \alpha^{2}}{2\left(1-\alpha^{2}\right)} \widetilde{X}_{f}^{\mathrm{v}}\right\|_{\Omega}^{2} \\
& -\frac{\left(1-2 \alpha^{2}\right)^{2}}{4\left(1-\alpha^{2}\right)}\left\|\widetilde{X}_{f}^{\mathrm{v}}\right\|_{\Omega}^{2}+\int \Omega\left(X_{h}, \widetilde{X}_{f}^{\mathrm{v}}\right) \\
\geq & -2 \alpha^{2}\left\|\widetilde{X}_{f}^{\mathrm{h}}\right\|_{\beta}^{2} . \\
E_{2}^{\prime \prime}= & \left(1-\alpha^{2}\right)\left\|\dot{x}^{\mathrm{h}}\right\|_{\omega}^{2}-\left(2-2 \alpha^{2}\right)\left\langle\dot{x}^{\mathrm{h}}, \widetilde{X}_{f}^{\mathrm{h}}\right\rangle_{\omega} \\
& +\int \Omega\left(\widetilde{X}_{f}^{\mathrm{h}}, J \dot{x}^{\mathrm{h}}\right)-\int \omega\left(X_{G}^{\mathrm{h}}, J \dot{x}^{\mathrm{h}}\right) \\
\geq & \frac{2\left(1-\alpha^{2}\right)}{3}\left\|\dot{x}^{\mathrm{h}}\right\|_{\beta}^{2}-\left(2-2 \alpha^{2}\right)\left\langle\dot{x}^{\mathrm{h}}, \widetilde{X}_{f}^{\mathrm{h}}\right\rangle_{\beta}-\int \pi^{*} \beta\left(X_{G}^{\mathrm{h}}, J \dot{x}^{\mathrm{h}}\right) \\
& -\left(1-\alpha^{2}\right) \int \Omega\left(\dot{x}^{\mathrm{h}}, \widetilde{X}_{f}^{\mathrm{h}}\right)+\alpha^{2} \int \Omega\left(\widetilde{X}_{f}^{\mathrm{h}}, \dot{x}^{\mathrm{h}}\right)-\int \Omega\left(X_{G}^{\mathrm{h}}, J \dot{x}^{\mathrm{h}}\right) \\
\geq & \frac{1-\alpha^{2}}{3}\left\|\dot{x}^{\mathrm{h}}\right\|_{\beta}^{2}-\left(2-2 \alpha^{2}\right)\left\langle\dot{x}^{\mathrm{h}}, \widetilde{X}_{f}^{\mathrm{h}}\right\rangle_{\beta}-6 m^{2}\left\|\widetilde{X}_{f}^{\mathrm{h}}\right\|_{\beta}^{2}-\frac{1}{24}\left\|\dot{x}^{\mathrm{h}}\right\|_{\beta}^{2} \\
& +\frac{1-\alpha^{2}}{3}\left\|\dot{x}^{\mathrm{h}}\right\|_{\beta}^{2}-\frac{1}{2} \int\left|\dot{x}^{\mathrm{h}}\right|_{\beta}\left|\widetilde{X}_{f}^{\mathrm{h}}\right|_{\beta}-\frac{m}{4} \int\left|\dot{x}^{\mathrm{h}}\right|_{\beta}\left|\widetilde{X}_{f}^{\mathrm{h}}\right|_{\beta} \\
\geq & \left(\frac{1-\alpha^{2}}{3}-\frac{1}{4}-\frac{1}{24}-\frac{m}{4}\right)\left\|\dot{x}^{\mathrm{h}}\right\|_{\beta}^{2}-\left(\frac{1}{4}+\frac{m}{4}+6 m^{2}\right)\left\|\widetilde{X}_{f}^{\mathrm{h}}\right\|_{\beta}^{2} \\
\geq & -\left(\frac{1}{4}+\frac{m}{4}+6 m^{2}\right)\left\|\widetilde{X}_{f}^{\mathrm{h}}\right\|_{\beta}^{2} .
\end{aligned}
$$

The inequalities involving $E_{2}^{\prime \prime}$ hold if $\left(1-\alpha^{2}\right) / 3-1 / 4-\frac{1}{24}-m / 4 \geq 0$ and if $\epsilon_{0}$ is small enough so that $\left\|\left.\Omega\right|_{H_{0}}\right\|_{\infty,\{S \leq 4\}} \leq 1 / 4$ and $|\cdot|_{\omega}^{2} \geq \frac{2}{3}|\cdot|_{\beta}^{2}$ on $E$. The inequality involving $E_{1}^{\prime \prime}$ holds if $\rho$ is small enough (determined by $\alpha$ ).

We finally obtain

$$
E^{\prime} \geq\left(\frac{3}{4}-5 \alpha^{2}-2 m-\frac{m}{4}-6 m^{2}\right)\left\|\widetilde{X}_{f}^{\mathrm{h}}\right\|_{\beta}^{2} \geq 0 .
$$

The last inequality holds if $m$ is small enough, provided $\alpha$ is also small enough.

The fact that equality in (5.11) is attained only if $x$ is a 1-periodic orbit of $h+G$ in a critical fiber of $\widetilde{f}$ is obvious from the fact that all the above inequalities have to be equalities. In particular, we must have $\widetilde{X_{f}} \circ x \equiv 0$.

\section{Transversality for split almost complex structures}

One crucial ingredient in the construction of the Floer complex associated to a vector field $Y$ and to an almost complex structure $J$ is the possibility 
to choose the pair $(Y, J)$ such that the linearized operator

$$
\begin{gathered}
D_{u}: W^{1, p}\left(\mathbb{R} \times \mathbb{S}^{1}, u^{*} T \widehat{E}\right) \longrightarrow L^{p}\left(\mathbb{R} \times \mathbb{S}^{1}, u^{*} T \widehat{E}\right), \\
\xi \longmapsto \nabla_{s} \xi+J(u) \nabla_{t} \xi+\nabla_{\xi} J(u) \cdot u_{t}-\nabla_{\xi} Y(u)
\end{gathered}
$$

is surjective for every finite energy solution of the equation

$$
u_{s}+J(u) \cdot u_{t}=Y(u) .
$$

In Floer's original setting one has $Y=J X_{H}$, with $H$ a given Hamiltonian, whereas in our setting $Y$ is a ( $s$-independent) vector field satisfying Definition 4.4.

Our definition of the Floer homology groups makes use of the fibered structure on $\widehat{E}$ only in order to prove the a priori $C^{0}$-bounds on the finite energy solutions of (6.1). The arguments developed in $[\mathbf{1 1}, \mathbf{1 2}, \mathbf{1 7}, \mathbf{2 7}]$ apply in order to show that, for a fixed choice of $Y$, transversality can be achieved by a generic choice of $J$ provided one allows the use of almost complex structures that are time-dependent. This is sufficient in order to define the Floer homology in the setting of the present paper. Nevertheless, in order to compute it by constructing a spectral sequence, one needs to establish transversality inside the smaller class of split almost complex structures whose horizontal component is time-independent. This requires a refinement of the above mentioned arguments, by allowing not only variations of the vertical almost complex structure, but also of the horizontal distribution as in $[\mathbf{2 3}, \S 8.2 \mathrm{sq}$.$] and [\mathbf{2 8}]$. The purpose of this section is to prove this refined version of transversality.

We denote by $V$ or Vert the vertical subbundle ker $\pi_{*} \subset T \widehat{E}$, whereas the horizontal subbundle $V^{\perp_{\Omega}}$ is denoted by $H_{0}$ or Hor. We denote by $\mathcal{J}_{\tau}^{\text {vert }}$ the space of smooth $\tau$-periodic almost complex structures on $V$ which are timeindependent and standard outside a compact set and which are compatible with $\Omega$ (see Definition 4.1). We denote by $\mathcal{J}_{B}$ the space of smooth timeindependent almost complex structures on $B$ which are compatible with $\beta$ and which satisfy the (NEGATIVITY) property of Definition 2.1. We endow $\mathcal{J}_{\tau}^{\text {vert }}$ and $\mathcal{J}_{B}$ with the $C^{\infty}$-topology. Given $J_{B} \in \mathcal{J}_{B}$, we denote by $\widetilde{J}_{B}^{H}$ its lift with respect to a given horizontal distribution $H$.

The connection 2 -form $\Omega$ can be perturbed while preserving at the same time closedness and keeping it unchanged along the fibers. We describe here a method borrowed from $[\mathbf{2 3}, \S 8.2]$, with the significant difference that we need to allow time-dependent perturbations of the horizontal distribution in order to achieve transversality.

The starting point is to consider on $B$ a 1 -form $H$ with values in the bundle $\mathcal{C}_{0}^{\infty}(\widehat{E})$ whose fiber at $z \in B$ is the space $C_{0}^{\infty}\left(\widehat{E}_{z}\right)$ of compactly supported smooth functions on $\widehat{E}_{z}$. We assume that $H$ is $\tau$-periodic and we use from now on the notation $H^{t}$ in order to express the dependence on $t$, with 
$H^{t}=H^{t+\tau}$. We denote the action of $H$ by

$$
\begin{gathered}
T_{z} B \longrightarrow C_{0}^{\infty}\left(\mathbb{S}_{\tau}^{1} \times \widehat{E}_{z}\right), \quad z \in B, \\
\zeta \longmapsto H_{\zeta}^{t},
\end{gathered}
$$

where $\mathbb{S}_{\tau}^{1}$ is the circle of length $\tau$. We define a time-dependent 1 -form $\sigma_{H}^{t} \in$ $\Omega^{1}(\widehat{E})$ by

$$
\sigma_{H}^{t}(x ; v)=H_{\zeta}^{t}(x), \quad \zeta=\pi_{*} v,
$$

where $x \in \widehat{E}$ and $v \in T_{x} \widehat{E}$. The 1 -form $\sigma_{H}^{t}$ vanishes on the fibers by definition. The connection 2 -form associated to $H^{t}$ is defined to be

$$
\Omega_{H}^{t}=\Omega-d \sigma_{H}^{t} .
$$

The horizontal subspace of $\Omega_{H}^{t}$ at $x \in \widehat{E}$ is

$$
\begin{aligned}
\operatorname{Hor}_{H^{t} ; x} & =\left\{v-X_{H_{\zeta}^{t}}(x): v \in \operatorname{Hor}_{x}, \quad \zeta=\pi_{*} v\right\} \\
& =\operatorname{graph}\left(-X_{H^{t}}: \operatorname{Hor}_{x} \longrightarrow \operatorname{Vert}_{x}, \quad v \longmapsto-X_{H_{\pi_{*}}^{t}}(x)\right) .
\end{aligned}
$$

In the above notation, $X_{H_{\zeta}^{t}}$ represents, for a given $z \in B$, the Hamiltonian vector field of the function $H_{\zeta}^{t}$ defined on $\widehat{E}_{z}$. We denote $\mathcal{H}_{\tau}=$ $\Omega^{1}\left(B, \mathcal{C}_{0}^{\infty}\left(\mathbb{S}_{\tau}^{1} \times \widehat{E}\right)\right)$ and, for a given compact set $N \subset \widehat{E}$, we let

$$
\mathcal{H}_{\tau}(N) \subset \mathcal{H}_{\tau}
$$

be the subspace of all those forms with support contained in $N$. The connection 2-form $\Omega^{t}$ is time-dependent but nevertheless constant on the fibers. For each curve in $B$, parallel transport along $H^{t}$ defines a path of symplectomorphisms between the fibers.

We use the shorthand notation $\widetilde{J}_{B}^{H}$ for the (time-dependent!) lift $\widetilde{J}_{B}^{\text {Hor }_{H} t}$ of an almost complex structure $J_{B}$ on the base. Any triple $\left(J_{V}, J_{B}, H\right) \in$ $\mathcal{J}_{\tau}^{\text {vert }} \times \mathcal{J}_{B} \times \mathcal{H}_{\tau}$ gives rise to an almost complex structure $J$ on $\widehat{E}$ defined as

$$
J=J_{V} \oplus \widetilde{J}_{B}^{H} .
$$

Let $J_{0}$ be the almost complex structure corresponding to the fixed triple $\left(J_{V}, J_{B}, 0\right)$. The action of the almost complex structures corresponding to triples $\left(J_{V}, J_{B}, H\right), H \in \mathcal{H}_{\tau}$ can be explicitly described (see $[\mathbf{2 3}]$ ) as

$$
J_{t} v=J_{0} v+J_{V, t} X_{H_{\pi_{*} v}^{t}}(x)-X_{H_{J_{B}}^{t} \pi_{*} v}(x), \quad v \in T_{x} \widehat{E} .
$$

Before stating our transversality result, we recall the following theorem of Salamon and Zehnder, which implies in particular transversality for all moduli spaces of Floer trajectories in the time-independent setting for symplectically aspherical manifolds. 
Theorem 6.1 ([27], Thm. 7.3). Let $(B, \beta)$ be a closed symplectic manifold such that

$$
\left\langle[\beta], \pi_{2}(B)\right\rangle=0 .
$$

Let $f: B \longrightarrow \mathbb{R}$ be a Morse function and $J_{B}$ a time-independent almost complex structure compatible with $\beta$, such that the flow of $\nabla^{J_{B}} f$ is MorseSmale. Let

$$
\begin{gathered}
D_{u, \tau}: W^{1, p}\left(\mathbb{R} \times \mathbb{S}_{\tau}^{1}, u^{*} T B\right) \longrightarrow L^{p}\left(\mathbb{R} \times \mathbb{S}_{\tau}^{1}, u^{*} T B\right), \\
\xi \longmapsto \nabla_{s} \xi+J_{B}(u) \nabla_{t} \xi+\nabla_{\xi} J_{B}(u) \cdot u_{t}-\nabla_{\xi} \nabla f
\end{gathered}
$$

be the linearization of Floer's equation

$$
u_{s}+J_{B}(u) u_{t}=\left(\nabla^{J_{B}} f\right) \circ u,
$$

defined for $\tau$-periodic maps $u: \mathbb{R} \times \mathbb{S}_{\tau}^{1} \longrightarrow B$. The following assertions hold if $\tau$ is small enough.

a) The operator $D_{u, \tau}$ is surjective for any solution $u: \mathbb{R} \longrightarrow B$ of (6.4) which is independent of $t$.

b) Every finite energy solution of (6.4) is independent of $t$.

Remark 6.2 (Reparametrizations). (i) The norm of $D_{u, \tau}$ does not depend on the parameter $\tau \in] 0, \tau_{0}$ ] because $D_{u, \tau} \xi=\nabla_{s} \xi-\nabla_{\xi} \nabla f$.

(ii) The statement of the above theorem remains true if we fix the period and allow the coefficient in front of $f$ to go to zero. The reason is that any $\tau$-periodic solution $u(s, t)$ gives rise to a $\tau_{0}$-periodic solution $u_{0}(s, t)=$ $u\left(\frac{\tau}{\tau_{0}} s, \frac{\tau}{\tau_{0}} t\right)$, which in turn satisfies the equation $\partial_{s} u_{0}+J_{B} \partial_{t} u_{0}=\frac{\tau}{\tau_{0}} \nabla f$.

We fix from now on a Morse function $f: B \longrightarrow \mathbb{R}$, an almost complex structure $J_{B} \in \mathcal{J}_{B}$ and a period $\tau>0$ such that the conclusions of Theorem 6.1 hold true.

If the almost complex structure $J_{B}$ satisfies the (NEGATIVITY) assumption of Definition 2.1, then for every $\left(J_{V}, H\right) \in \mathcal{J}_{\tau}^{\text {vert }} \times \mathcal{H}_{\tau}$ there exists $\epsilon_{0}>0$ such that

$$
\omega_{\epsilon}=\pi^{*} \beta+\epsilon \Omega
$$

tames

$$
J=J_{V} \oplus \widetilde{J}_{B}^{H}
$$

for all $0<\epsilon \leq \epsilon_{0}$. Following the previous section, we consider Hamiltonians of the form

$$
K=h+\widetilde{f}+G
$$

The function $h=h(S)$ is convex and linear for $S \geq 1$ with slope $\lambda_{\max }$ satisfying

$$
\frac{\tau}{\epsilon_{0}} \cdot \lambda_{\max } \notin \operatorname{Spec}(\partial E) .
$$

The function $G$ is a $\tau$-periodic perturbation localized in a neighborhood of the critical fibers of $\widetilde{f}=\pi \circ f$. More precisely, we denote the critical points 
of $f$ by $p_{i}, 1 \leq i \leq \ell$, we fix open neighborhoods $p_{i} \in V_{i} \Subset U_{i}$ such that $U_{i} \cap U_{j}=\emptyset, i \neq j$ and we require that $\operatorname{supp}(G) \subset E \cap \bigcup_{p_{i} \in \operatorname{Crit}(f)} \pi^{-1}\left(V_{i}\right)$. We denote

$$
N=E \cap{ }^{c} \pi^{-1}\left(\bigcup U_{i}\right)
$$

so that $N \cap \operatorname{supp}(G)=\emptyset$. Given $h$ we choose $G$ and $\epsilon_{0}$ such that the $\tau$-periodic orbits of $K$ with respect to $\omega_{\epsilon_{0}}$ are nondegenerate and lie in the critical fibers of $\tilde{f}=\pi \circ f$, while the vector field

$$
\begin{aligned}
& \mathcal{Y}^{\epsilon_{0}}(t, x)=J_{t} \dot{x}-Y^{\epsilon_{0}}(t, x), \\
& Y^{\epsilon_{0}}(t, x)=J_{t}\left(X_{\epsilon_{0} h}^{\epsilon_{0}}+\epsilon_{0} \widetilde{X_{f}}+\left(X_{\epsilon_{0} G}^{\epsilon_{0}}\right)^{\mathrm{v}}(t, x)\right)
\end{aligned}
$$

is a negative pseudo-gradient for the action functional $A_{\epsilon_{0} K}^{\epsilon_{0}}$ defined on the space of contractible $\tau$-periodic loops in $\widehat{E}$. The superscript $\epsilon_{0}$ indicates, as usual, the fact that the Hamiltonian vector fields and the symplectic action are computed with respect to the form $\omega_{\epsilon_{0}}$.

Remark 6.3 (Uniform upper bound for $\epsilon$ ). The rescaling parameter $\epsilon$ is allowed to vary in some interval $\left.] 0, \epsilon_{0}\right]$ with $\epsilon_{0}$ small enough in order to ensure nondegeneracy and taming for $\omega_{\epsilon}=\pi^{*} \beta+\epsilon \Omega$. The only other point where we use the rescaling $\Omega \rightsquigarrow \epsilon \Omega$ is in the proof of the pseudo-gradient property for loops contained in $E$ in Proposition 5.8, where one might need to further diminish the constant $\epsilon_{0}$, depending on $\left\|\left.G\right|_{E}\right\|_{C^{1}}$. Since the latter quantity can be uniformly bounded independently of the choice of $h$, we conclude that we can construct an admissible cofinal family of Hamiltonians $K$ admitting a uniform constant $\epsilon_{0}$.

Given a Hamiltonian $K$ and a parameter $0<\epsilon \leq \epsilon_{0}$ as in Remark 6.3, we define the space of regular vertical almost complex structures and Hamiltonian perturbations

$$
\left(\mathcal{J}_{\tau}^{\mathrm{vert}} \times \mathcal{H}_{\tau}\right)^{\mathrm{reg}}(\epsilon, K) \subset \mathcal{J}_{\tau}^{\mathrm{vert}} \times \mathcal{H}_{\tau}(N)
$$

as consisting of pairs $\left(J_{V}, H\right) \in \mathcal{J}_{\tau}^{\text {vert }} \times \mathcal{H}_{\tau}(N)$ such that, for every finite energy solution $u: \mathbb{R} \times \mathbb{S}_{\tau}^{1} \longrightarrow \widehat{E}$ of the equation

$$
u_{s}+J u_{t}=Y^{\epsilon} \circ u
$$

the linearized operator

$$
\begin{gathered}
D_{u, \tau}^{\epsilon}: W^{1, p}\left(\mathbb{R} \times \mathbb{S}_{\tau}^{1}, u^{*} T \widehat{E}\right) \longrightarrow L^{p}\left(\mathbb{R} \times \mathbb{S}_{\tau}^{1}, u^{*} T^{*} \widehat{E}\right), \\
\xi \longmapsto \nabla_{s}^{\epsilon} \xi+J(u) \nabla_{t}^{\epsilon} \xi+\nabla_{\xi}^{\epsilon} J(u) \cdot u_{t}-\nabla_{\xi}^{\epsilon} Y^{\epsilon}(u)
\end{gathered}
$$

is surjective. The connection $\nabla^{\epsilon}$ is the Levi-Civita connection associated to the metric defined by $\omega_{\epsilon}$ and $J=J_{V} \oplus \widetilde{J}_{B}^{H}$.

Notation. Given a map $u: \mathbb{R} \times \mathbb{S}_{\tau}^{1} \longrightarrow \widehat{E}$, we denote its projection by

$$
v=\pi \circ u \text {. }
$$


Given $J_{V} \in \mathcal{J}_{\tau}^{\text {vert }}$, we denote by $\mathcal{J}_{\tau}^{\text {vert }}\left(J_{V}\right)$ the space of vertical almost complex structures which coincide with $J_{V}$ outside a compact set.

Remark 6.4 (Geometric property of the pseudo-gradient equation). The fundamental property of equation (6.5) is that the projected solutions $v=\pi \circ u$ satisfy the equation

$$
v_{s}+J_{B}(v) v_{t}=\epsilon \nabla^{J_{B}} f(v),
$$

for which transversality is ensured by Theorem 6.1. This geometric property plays a crucial role not only in the construction of the spectral sequence, but also in the proof of transversality within the class of split almost complex structures of the type $J_{V} \oplus \widetilde{J}_{B}^{H}$.

The aim of this section is to prove the following result.

Theorem 6.5 (Split transversality). Let $K, \epsilon_{0}$ be as above and $J_{V, 0} \in$ $\mathcal{J}_{\tau}^{\text {vert }}$.

a) There exist a positive constant $\left.\epsilon(K) \in] 0, \epsilon_{0}\right]$ and an open neighborhood $\mathcal{O}$ of $\left(J_{V, 0}, H_{0}\right)$ in $\mathcal{J}_{\tau}^{\text {vert }}\left(J_{V, 0}\right) \times \mathcal{H}_{\tau}(N)$ such that the operator

$$
\begin{gathered}
F_{u, \tau}^{\epsilon}: W^{1, p}\left(\mathbb{R} \times \mathbb{S}_{\tau}^{1}, v^{*} T B\right) \longrightarrow L^{p}\left(\mathbb{R} \times \mathbb{S}_{\tau}^{1}, v^{*} T B\right), \\
F_{u, \tau}^{\epsilon}(\xi)=\pi_{*}\left(D_{u, \tau}^{\epsilon} \cdot \widetilde{\xi}\right)
\end{gathered}
$$

is surjective for any finite energy solution $u$ of (6.5) with $0<\epsilon \leq \epsilon(K)$ and $\left(J_{V}, H\right) \in \mathcal{O}$;

b) For every $0<\epsilon \leq \epsilon(K)$, the set $\left(\mathcal{J}_{\tau}^{\text {vert }} \times \mathcal{H}_{\tau}\right)^{\text {reg }}(\epsilon, K) \cap \mathcal{O}$ is dense and of second Baire category in $\mathcal{O}$.

Remark 6.6 (On the parameter $\epsilon(K)$ ). The parameter $\epsilon(K)$ depends actually on the asymptotic slope of $K$. Indeed, it will be appearant from the proof that $\epsilon(K)$ depends on the $C^{0}$-bound for solutions of $u_{s}+J u_{t}=Y^{\epsilon} \circ u$ through the use of Lemma 6.7 below. These bounds, in turn, depend via Lemma 4.16 on the maximal difference between the actions of two closed orbits, i.e., on the asymptotic slope of $K$.

Proof. Let $\mathcal{O}$ be a neighborhood of $\left(J_{V, 0}, H_{0}\right)$ such that the following hold for $\left(J_{V}, H\right) \in \mathcal{O}$ :

- the almost complex structures $J=J_{V} \oplus \widetilde{J}_{B}^{H}$ are tamed by $\omega_{\epsilon}, 0<$ $\epsilon \leq \epsilon_{0}$

- finite energy solutions of $u_{s}+J u_{t}=Y^{\epsilon} \circ u$ admit a common uniform $C^{0}$-bound for $0<\epsilon \leq \epsilon_{0}$. This can be achieved as a consequence of the following two observations which slightly generalize the proof of Theorem 4.6. First, it is clear that, for $\epsilon>0$ fixed, one can allow the almost complex structure to slightly vary inside a compact set. Secondly, as $\epsilon>0$ varies, the vertical part of the vector field $Y^{\epsilon}$ remains unchanged, whereas the horizontal part is rescaled by $\epsilon$, so that assumptions (4.21-4.23) in Definition 4.4 are still satisfied. 
Lemma 6.7. There exist constants $c(\epsilon), 0<\epsilon \leq \epsilon_{0}$ with $c(\epsilon) \longrightarrow 0, \epsilon \longrightarrow 0$ such that, for any finite energy solution $u$ of equation (6.5) with $\left(J_{V}, H\right) \in$ $\mathcal{O}$, we have

$$
\left\|\left|F_{u, \tau}^{\epsilon}-D_{v} \|\right| \leq c(\epsilon) .\right.
$$

Proof. The Levi-Civita connection $\nabla$ associated to a metric $\langle\cdot, \cdot\rangle$ can be expressed as follows:

$$
\begin{aligned}
\left\langle\nabla_{Y} X, Z\right\rangle= & \frac{1}{2}\{X\langle Y, Z\rangle+Y\langle Z, X\rangle-Z\langle X, Y\rangle \\
& -\langle[X, Z], Y\rangle-\langle[Y, Z], X\rangle-\langle[X, Y], Z\rangle\} .
\end{aligned}
$$

By applying the above formula to the connection $\nabla^{\epsilon}$ associated to the (timedependent) metric $g_{\epsilon}(v, w)=\frac{1}{2}\left(\omega_{\epsilon}(v, J w)+\omega_{\epsilon}(w, J v)\right)$ one sees that, for any two vector fields $X \in \mathcal{X}(\widehat{E})$ and $Y \in \mathcal{X}(B)$, we have

$$
\begin{aligned}
& \left(\nabla_{\widetilde{Y}}^{\epsilon} X\right)^{\mathrm{h}} \longrightarrow \widetilde{\nabla_{Y} \pi_{*} X}, \\
& \left(\nabla_{X}^{\epsilon} \tilde{Y}\right)^{\mathrm{h}} \longrightarrow \widetilde{\nabla_{\pi_{*} X} Y}, \quad \epsilon \longrightarrow 0,
\end{aligned}
$$

where $\nabla$ is the Levi-Civita connection on $B$ corresponding to the metric $g_{B}(\cdot, \cdot)=\beta\left(\cdot, J_{B} \cdot\right)$. The convergence is uniform on every compact set. More precisely, for any compact set $\mathcal{K} \subset \widehat{E}$, we have

$$
\begin{gathered}
\left\|\left(\nabla_{\widetilde{Y}}^{\epsilon} X\right)^{\mathrm{h}}-\widetilde{\nabla_{Y} \pi_{*} X}\right\|_{g_{B}} \leq c_{1}(\epsilon, \mathcal{K})\|X\|_{C^{1}\left(g_{\epsilon_{0}}\right)}\|Y\|_{C^{0}}, \\
\left\|\left(\nabla_{X}^{\epsilon} \widetilde{Y}\right)^{\mathrm{h}}-\widetilde{\nabla_{\pi_{*} X} Y}\right\|_{g_{B}} \leq c_{1}(\epsilon, \mathcal{K})\|X\|_{C^{0}\left(g_{\epsilon_{0}}\right)}\|Y\|_{C^{1}},
\end{gathered}
$$

with $c_{1}(\epsilon, \mathcal{K}) \longrightarrow 0, \epsilon \longrightarrow 0$. Similarly, we have

$$
\left\|\left(\nabla_{\widetilde{Y}}^{\epsilon} J\right)^{\mathrm{h}}-\widetilde{\nabla_{Y} J_{B}}\right\|_{g_{B}} \leq c_{2}(\epsilon, \mathcal{K})\|Y\|_{C^{0}\left(g_{B}\right)},
$$

with $c_{2}(\epsilon, \mathcal{K}) \longrightarrow 0, \epsilon \longrightarrow 0$. The estimates (6.8-6.10), together with the explicit form of the operators involved and the existence of the uniform $C^{0}$ bound on solutions of Floer's equation (6.5) for $\left(J_{V}, H\right) \in \mathcal{O}$ and $0<\epsilon \leq \epsilon_{0}$, imply the conclusion.

Lemma 6.8. The operators $D_{v}$, where $v$ runs over all Floer (timeindependent) trajectories on $B$ corresponding to $f$ and $J_{B}$, admit uniformly bounded right inverses.

Proof. This is a reformulation of the gluing theorem for Floer trajectories in the transverse case (see, for example, $[\mathbf{2 6}]$ for the latter). The key step in the gluing construction is to prove that the linearized operator is surjective along preglued curves and that it admits a right inverse which is uniformly bounded for large enough values of the gluing parameter. This implies that one can find uniformly bounded right inverses for the operator $D_{v}$ when $v$ belongs to a small neighborhood of the boundary of the moduli space of trajectories. Such a uniform bound can clearly be found on the remaining 
relatively compact set contained in the interior of the moduli space of trajectories, and gives the existence of uniformly bounded right inverses for $D_{v}$ for any choice of $v$.

We prove now assertion (a) in the theorem. Let $C$ be the uniform upper bound provided by Lemma 6.8 and choose $\epsilon(K)$ small enough so that the constant $c(\epsilon)$ in Lemma 6.7 satisfies $c(\epsilon)<1 / 2 C$ for $0<\epsilon \leq \epsilon(K)$. Given a finite energy solution $u$, let $Q_{v}$ be a right inverse for $D_{v}, v=\pi \circ u$ such that $\left\|Q_{v}\right\| \leq C$. Then

$$
\left\|F_{u, \tau}^{\epsilon} Q_{v}-\operatorname{Id}\right\|=\left\|F_{u, \tau}^{\epsilon} Q_{v}-D_{v} Q_{v}\right\| \leq 1 / 2,
$$

hence the operator $F_{u, \tau}^{\epsilon} Q_{v}$ is invertible and the norm of its inverse is $\leq$ 2. Then $Q_{v}\left(F_{u, \tau}^{\epsilon} Q_{v}\right)^{-1}$ is a right inverse for $F_{u, \tau}^{\epsilon}$ of norm $\leq 2 C$, and in particular $F_{u, \tau}^{\epsilon}$ is surjective.

We prove now assertion (b). We follow the proof of Theorem 5.1 in $[\mathbf{1 2}]$ in particular, it is enough to obtain the conclusion when $\mathcal{J}_{\tau}^{\text {vert }}\left(J_{V, 0}\right) \times \mathcal{H}_{\tau}(N)$ is endowed with the $C^{\ell}$-topology, $\ell \geq 1$.

We fix $0<\epsilon \leq \epsilon(K)$. The key step is to prove that, for any $\tau$-periodic orbits $x^{-}, x^{+}$of $K$, the universal moduli space

$$
\mathcal{M}\left(x^{-}, x^{+}, \mathcal{O}\right)=\left\{\left(u, J_{V}, H\right): \bar{\partial}_{J_{V} \oplus \widetilde{J}_{B}^{H}, Y^{\epsilon}} u=0\right\}
$$

is a Banach manifold. The universal moduli space is naturally the zero set of the section

$$
\mathcal{F}: \mathcal{B} \times \mathcal{O} \longrightarrow \mathcal{E}, \quad \mathcal{F}\left(u, J_{V}, H\right)=\bar{\partial}_{J_{V} \oplus \widetilde{J}_{B}^{H}, Y^{\epsilon}} u .
$$

Here $\mathcal{B}=\mathcal{B}\left(x^{-}, x^{+}\right)$is the space of continuous maps $u: \mathbb{R} \times \mathbb{S}_{\tau}^{1} \longrightarrow \widehat{E}$ which are locally of class $W^{1, p}$ and which converge to $x^{-}, x^{+}$as $s \longrightarrow \pm \infty$ with a suitable exponential decay condition at infinity [12], while $\mathcal{E}$ is the Banach bundle whose fiber at $\left(u, J_{V}, H\right)$ is $L^{p}\left(u^{*} T \widehat{E}\right)$.

We need to prove that $\mathcal{F}$ is transverse to the zero section of $\mathcal{E}$. We denote by $\pi$ the vertical projection $T_{\left(\left(u, J_{V}, H\right), 0\right)} \mathcal{E} \longrightarrow \mathcal{E}_{\left(u, J_{V}, H\right)}$. The vertical differential

$$
D \mathcal{F}\left(u, J_{V}, H\right)=\pi \circ d \mathcal{F}\left(u, J_{V}, H\right)
$$

is given at a solution $u$ of (6.5) by

$$
D \mathcal{F}\left(u, J_{V}, H\right) \cdot(\xi, Z, h)=D_{u} \xi+Z_{t}(u) J_{V} u_{s}^{\text {vert }}+X_{h_{* *} u_{s}^{\text {horiz }}} \cdot
$$

Here $\xi \in T_{u} \mathcal{B}=W^{1, p}\left(u^{*} T \widehat{E}\right)$ and $h \in T_{H} \mathcal{H}_{\tau}(N)=\mathcal{H}_{\tau}(N)$. The tangent vector $Z \in T_{J_{V}} \mathcal{J}_{\tau}^{\text {vert }}\left(J_{V, 0}\right)$ is a $C^{\ell}$-map $\mathbb{S}_{\tau}^{1} \times T \widehat{E} \longrightarrow T \widehat{E}$ which has compact support in $\widehat{E}$ and satisfies

$$
J_{V}^{t} Z_{t}+Z_{t} J_{V}^{t}=0, \quad \Omega\left(Z_{t} v, w\right)+\Omega\left(v, Z_{t} w\right)=0, v, w \in \text { Vert. }
$$


Let us explain the term $X_{h_{\pi_{*} \partial_{s} u}}$ in (6.11). We need to study the change in

$$
\bar{\partial}_{J_{V} \oplus \widetilde{J}_{B}^{H}, Y^{\epsilon}} u=u_{s}+\left(J_{V} \oplus \widetilde{J}_{B}^{H}\right) u_{t}-\left(J_{V} \oplus \widetilde{J}_{B}^{H}\right)\left(X^{\mathrm{v}}+\epsilon \widetilde{X}_{f}{ }^{H}\right)
$$

as we replace $H$ by $H+h$. By (6.3), we obtain

$$
\begin{aligned}
\left(J_{V} \oplus \widetilde{J}_{B}^{H+h}\right) \cdot \widetilde{X}_{f}^{H+h} & =\left(J_{V} \oplus \widetilde{J}_{B}^{H+h}\right) \cdot\left(\widetilde{X}_{f}^{H}-X_{h_{X_{f}}}\right) \\
& =-J_{V} X_{h_{X_{f}}}+\left(J_{V} \oplus \widetilde{J}_{B}^{H}\right) \cdot \widetilde{X}_{f}^{H}+J_{V} X_{h_{X_{f}}}-X_{h_{J_{B} X_{f}}} \\
& =\left(J_{V} \oplus \widetilde{J}_{B}^{H}\right) \cdot \widetilde{X}_{f}^{H}-X_{h_{J_{B} X_{f}}}
\end{aligned}
$$

and

$$
\left(J_{V} \oplus \widetilde{J}_{B}^{H+h}\right) \cdot u_{t}=\left(J_{V} \oplus \widetilde{J}_{B}^{H}\right) \cdot u_{t}+J_{V} X_{h_{\pi_{*} u_{t}}}-X_{h_{J_{B} \pi_{*} u_{t}}} .
$$

Since $J_{B} \pi_{*} u_{t}-\epsilon J_{B} X_{f}=\pi_{*} u_{s}$, we infer that the variation in the direction $h$ of $\bar{\partial}_{J_{V} \oplus \widetilde{J}_{B}^{H}, Y^{\epsilon}} u$ is

$$
\delta \bar{\partial} \cdot h=J_{V} X_{h_{*} u_{t}}+X_{h_{*} u_{s}} .
$$

On the other hand, $\pi_{*} u_{t}$ vanishes because the projected trajectories on the base are time-independent, hence $\delta \bar{\partial} \cdot h=X_{h_{\pi_{*} u_{s}}}$.

We need to show that $D \mathcal{F}$ is onto for any $\left(u, J_{V}, H\right) \in \mathcal{M}\left(x^{-}, x^{+}, \mathcal{O}\right)$. The operator $D_{u}$ is Fredholm and the same holds for $D \mathcal{F}$. In particular im $(D \mathcal{F})$ is closed and, in order to prove surjectivity, it is enough to prove that $D \mathcal{F}$ has a dense range. Equivalently, we have to show that the annihilator

$$
A=\left\{\eta \in L^{q}\left(u^{*} T \widehat{E}\right): \iint_{\mathbb{R} \times \mathbb{S}_{\tau}^{1}}\left\langle\eta, D \mathcal{F}\left(u, J_{V}, H\right) \cdot(\xi, Z, h)\right\rangle d s d t=0, \forall \xi, Z, h\right\}
$$

is zero, where $1 / p+1 / q=1$. For $\eta \in A$, we must have

$$
\begin{aligned}
\iint\left\langle\eta, D_{u} \xi\right\rangle d s d t & =0, \\
\iint\left\langle\eta, Z_{t}(u) J_{V} u_{s}^{\mathrm{vert}}\right\rangle d s d t & =0, \\
\iint\left\langle\eta, X_{h_{*} u_{s}^{\text {horiz }}}\right\rangle d s d t & =0
\end{aligned}
$$

for all $\xi \in W^{1, p}\left(u^{*} T \widehat{E}\right), Z \in T_{J_{V}} \mathcal{J}_{\tau}^{\text {vert }}\left(J_{V, 0}\right), h \in \mathcal{H}_{\tau}(N)$. Condition (6.12) states that $\eta$ is a weak solution of $D_{u}^{*} \eta=0$, where $D_{u}^{*}$ is the formal adjoint of $D_{u}$ which is obtained by formally replacing in $D_{u}$ the term $\nabla_{s}$ with $-\nabla_{s}$. Elliptic regularity implies that $\eta$ is of class $C^{\ell}$ and is a strong solution of $D_{u}^{*} \eta=0$, while unique continuation (see [12]) ensures that it is enough to show that $\eta$ vanishes on an open set in order to obtain global vanishing. We denote $\eta=\eta^{\mathrm{h}}+\eta^{\mathrm{v}}$ the decomposition of $\eta$ into horizontal and vertical parts with respect to the splitting $T \widehat{E}=\operatorname{Vert} \oplus \operatorname{Vert}^{\perp_{\Omega}}$. In general, the 
(time-dependent) decomposition of a vector $X$ with respect to the splitting $T \widehat{E}=\operatorname{Vert} \oplus \operatorname{Hor}_{H}$ is denoted $X=X^{\text {vert }}+X^{\text {horiz }}$.

We first show that $\eta^{\mathrm{v}} \equiv 0$ on some open set $U$. The special form of our Floer equation separates nonconstant trajectories $u$ in two classes: those entirely contained in a fiber, and those satisfying $\pi_{*} u_{s}=v_{s} \neq 0$ running from one fiber to another.

Let $u$ be a nonconstant trajectory contained in a fiber. One of the fundamental results of $[\mathbf{1 2}]$ states that the set

$R(u)=\left\{(s, t) \in \mathbb{R} \times \mathbb{S}_{\tau}^{1}: u_{s}(s, t) \neq 0, u(s, t) \neq x^{ \pm}(t), u(s, t) \notin u(\mathbb{R}-\{s\}, t)\right\}$

of regular points is open and dense in $\mathbb{R} \times \mathbb{S}_{\tau}^{1}$. We claim that $\eta^{\mathrm{v}} \equiv 0$ on $R(u)$, hence by density $\eta^{\mathrm{v}} \equiv 0$. Assume by contradiction that this is not the case and $\eta^{\mathrm{v}}(s, t) \neq 0$ for some $(s, t) \in R(u)$, hence on a small neighborhood of $(s, t)$. Because $u_{s}^{\mathrm{v}}=u_{s}=u_{s}^{\text {vert }}$, we can choose, according to [12], a time-dependent tangent vector $Z_{t}$ such that $\iint_{\mathbb{R} \times \mathbb{S}_{\tau}^{1}}\left\langle\eta, Z_{t}(u) J_{V} u_{s}^{\text {vert }}\right\rangle>0$, contradicting (6.13).

Let now $u$ be a nonconstant trajectory running from one fiber to another. In this case, we have $R(u)=\mathbb{R} \times \mathbb{S}_{\tau}^{1}$. We distinguish two situations: either $u$ crosses $N$, or not. In the second case, the part of $u$ projecting onto $B \backslash \bigcup_{i} U_{i}$ must live in $\{S \geq 1\}$, and we deduce the existence of a point $(s, t)$ such that $u(s, t) \in E \backslash N$ and $u_{s}^{\mathrm{vert}}(s, t)=u_{s}^{\mathrm{v}}(s, t) \neq 0$. Then we can use again condition (6.13) in order to show that $\eta^{\mathrm{v}}$ has to vanish in a neighborhood $U$ of $(s, t)$. In the first case, we use (6.14) in order to show that $\eta^{\mathrm{v}}$ vanishes on $U=u^{-1}(\operatorname{im} u \cap \operatorname{int} N)$. By contradiction, let $(s, t)$ be a point where $\eta^{\mathrm{v}}(s, t) \neq 0$. We know that $\pi_{*} u_{s}=v_{s} \neq 0$, hence we can choose a time-dependent tangent vector $h$ with support in $N$ such that $\iint_{\mathbb{R} \times \mathbb{S}_{\tau}^{1}}\left\langle\eta, X_{h_{* * u_{s}}}\right\rangle>0$, contradicting (6.14).

Let us now prove that $\eta^{\mathrm{h}}$ also vanishes on some nonempty open set $V \subset U$, knowing that $\eta^{\mathrm{v}}$ vanishes on $U$. Let $\beta: \mathbb{R} \times \mathbb{S}_{\tau}^{1} \longrightarrow \mathbb{R}_{+}$be a positive smooth function supported in $U$ which is not identically zero. We claim that $\eta^{\mathrm{h}}$ vanishes on the open set $\{z \in U: \beta(z)>0\}$. By contradiction, let us assume that this is not the case. The projection $\pi_{*}(\beta \eta)=\pi_{*}\left(\beta \eta^{\mathrm{h}}\right)$ is an $(s, t)$-dependent vector field along $v=\pi_{*} u$, supported in $U$ (note that $v$ may as well be constant, but this does not interfere with the argument). We have seen that the operator $F_{u, \tau}^{\epsilon}=\pi_{*} \circ D_{u} \circ \sim$ is surjective, hence there exists $\xi \in W^{1, p}\left(v^{*} T B\right)$ such that $\left(D_{u} \cdot \widetilde{\xi}\right)^{\mathrm{h}}=\beta \eta^{\mathrm{h}}$. We now use $\eta^{\mathrm{v}} \equiv 0$ on $U$ in order to obtain

$$
\iint_{\mathbb{R} \times \mathbb{S}_{\tau}^{1}}\left\langle\eta, D_{u} \widetilde{\xi}\right\rangle=\iint_{\mathbb{R} \times \mathbb{S}_{\tau}^{1}}\langle\eta, \beta \eta\rangle=\iint_{U}\left\langle\eta^{\mathrm{h}}, \beta \eta^{\mathrm{h}}\right\rangle>0
$$

which is a contradiction with (6.12). 
We have therefore proved that $\eta$ vanishes on a nonempty open set $V \subset$ $\mathbb{R} \times \mathbb{S}_{\tau}^{1}$. By unique continuation, $\eta$ vanishes identically on $\mathbb{R} \times \mathbb{S}_{\tau}^{1}$, and this finishes the proof of Theorem 6.5.

\section{The spectral sequence}

7.1. General formalism. We recall some relevant notions concerning spectral sequences in order to fix notation, and we refer to $[\mathbf{1}, \mathbf{2 1}]$ for details. For our purposes, a spectral sequence is a sequence of bigraded differential modules $\left(E_{r}^{p, q}, d_{r}\right)$ associated to a graded differential complex $\left(C=\oplus_{k \geq 0} C^{k}, \partial\right)$ endowed with a filtration

$$
C^{k}=F_{0} C^{k} \supset F_{1} C^{k} \supset \cdots \supset F_{n} C^{k} \supset 0 .
$$

Saying that $F_{p} C, p \geq 0$ defines a filtration means that $\partial\left(F_{p} C^{k}\right) \subset F_{p} C^{k+1}$, where we assume that the differential $\partial$ has degree +1 . The differential $d_{r}$ has bidegree $(r,-r+1)$. The main feature of a spectral sequence is that $E_{r+1}=H\left(E_{r}, d_{r}\right), r \geq 0$, with $E_{0}^{p, q}=F_{p} C^{p+q} / F_{p+1} C^{p+q}$. Within the above setup, the groups $E_{r}^{p, q}$ stabilize and the limit $E_{\infty}^{p, q}$ satisfies $E_{\infty}^{p, q}=$ $F_{p} H^{p+q} / F_{p+1} H^{p+q}$ for some filtration $F_{p} H^{k}, p \geq 0$ on the cohomology $H=$ $H(C, \partial)$. Spectral sequences are functorial in the sense that a morphism of filtered complexes induces a morphism between the associated spectral sequences.

Example 7.1. We assume $C^{k}=\oplus_{p=0}^{n} C_{p}^{k}$ and $\partial=\partial_{0}+\partial_{1}+\cdots+\partial_{n}$, with $\partial_{r}: C_{p}^{k} \longrightarrow C_{p+r}^{k+1}$. We denote $C_{p}=\oplus_{k \geq 0} C_{p}^{k}$ and $F_{p} C^{k}=\oplus_{s \geq p} C_{s}^{k}$, so that $F_{p} C^{k}$ defines a filtration. We then have $E_{0}^{p, q}=C_{p}^{p+q}$ and

$$
\begin{gathered}
E_{1}=\oplus_{p} H\left(C_{p}, \partial_{0}\right), \\
d_{1}\left(\left[\alpha_{p}\right]\right)=\left[\partial_{1} \alpha_{p}\right] \in H\left(C_{p+1}, \partial_{0}\right), \quad\left[\alpha_{p}\right] \in H\left(C_{p}, \partial_{0}\right) .
\end{gathered}
$$

More generally, let $D_{r}=\partial_{0}+\partial_{1}+\cdots+\partial_{r}, r \geq 0$. An element $\alpha_{p} \in C_{p}$ defines a class in $E_{r}$ if and only if there exist $\beta_{p+i} \in C_{p+i}, 1 \leq i \leq r-1$ such that $D_{r-1} \alpha_{p}+D_{r-2} \beta_{p+1}+\cdots+D_{0} \beta_{p+r-1}=0$, and the differential $d_{r}$ acts as

$$
\begin{aligned}
d_{r}\left[\alpha_{p}\right] & =\left[\partial_{r} \alpha_{p}+\partial_{r-1} \beta_{p+1}+\cdots+\partial_{1} \beta_{p+r-1}\right] \\
& =\left[D_{r} \alpha_{p}+D_{r-1} \beta_{p+1}+\cdots+D_{1} \beta_{p+r-1}\right] .
\end{aligned}
$$

\subsection{Morse homology and local systems of coefficients.}

7.2.1. Morse homology with values in a local system of coefficients. The formalism of local coefficients for singular or cellular homology was introduced by Steenrod [30]. This is our main reference for this section, together with McCleary [21]. The adaptation to Morse homology is straightforward but, to our knowledge, has not appeared previously in the literature. 
Definition 7.2. Let $R$ be a ring and $M$ be an $R$-module. A local system of coefficients with fiber $M$ on a topological space $B$ consists of the following data:

(1) one copy $M_{x}$ of $M$ for each $x \in B$, called the fiber at $x$;

(2) a family of isomorphisms $\left(\Phi_{\alpha}: M_{\alpha(0)} \stackrel{\sim}{\longrightarrow} M_{\alpha(1)}\right)_{\alpha \in \mathcal{P}(B)}$, where $\mathcal{P}(B)$ is the set of continuous paths in $B$, such that:

- if $\alpha \simeq \beta$ are homotopic with fixed endpoints, then $\Phi_{\alpha}=\Phi_{\beta}$;

- if $\alpha, \beta \in \mathcal{P}(B)$ satisfy $\alpha(1)=\beta(0)$, then $\Phi_{\alpha \cdot \beta}=\Phi_{\alpha} \circ \Phi_{\beta}$.

We call $\Phi_{\alpha}$ the parallel transport along $\alpha$.

\section{Remarks.}

1. Isomorphism classes of local systems of coefficients on a manifold are in one-to-one correspondence with isomorphism classes of locally constant sheaves.

2. If $B$ is simply-connected, then all local systems having the same fiber are isomorphic. More generally, the choice of a basepoint $x_{0} \in B$ and of a collection of paths connecting $x_{0}$ to $x \in B, x \neq x_{0}$, determines a one-to-one correspondence between local systems with fiber $M$ and representations $\pi_{1}\left(B, x_{0}\right) \longrightarrow \operatorname{Aut}\left(M_{x_{0}}\right)$. We call the representation associated to a local system $\mathcal{S}$ the monodromy representation of $\mathcal{S}$. It is well defined up to conjugation by an element of $\operatorname{Aut}\left(M_{x_{0}}\right)$.

We define the cohomology groups of $B$ with values in the local system $\mathcal{S}$, denoted by $H^{*}(B ; \mathcal{S})$, as the cohomology groups of $B$ with values in the associated locally constant sheaf $\mathcal{S}$, and we refer to [30] for a description in terms of singular cochains with coefficients.

Example 7.3 (Second term in the Leray-Serre spectral sequence). Let $F \hookrightarrow E \stackrel{\pi}{\longrightarrow} B$ be a locally trivial fibration. For any $q \geq 0$, we define a local system $\mathcal{H}^{q}(F, \partial F)$ with fiber $H^{q}(F, \partial F)$ as follows. The fiber at $x \in B$ is $H^{q}\left(F_{x}, \partial F_{x}\right)$. For a path $\alpha$ contained in a contractible open set $U \subset B$, we define $\Phi_{\alpha}=i_{\alpha(1)}^{*} i_{\alpha(0)}^{*}$, where $F_{\alpha(0)} \stackrel{i_{\alpha(0)}}{\hookrightarrow} \pi^{-1}(U) \stackrel{i_{\alpha(1)}}{\longleftrightarrow} F_{\alpha(1)}$ are the inclusions, inducing isomorphisms in cohomology. This isomorphism is independent of $U$ as long as the latter is contractible. For a path $\alpha \in$ $\mathcal{P}(B)$, we consider a subdivision $0=t_{0}<t_{1}<\cdots<t_{N}<t_{N+1}=1$ such that $\alpha_{i}=\left.\alpha\right|_{\left[t_{i}, t_{i+1}\right]}$ is contained in a contractible open set and define $\Phi_{\alpha}=\Phi_{\alpha_{N}} \circ \Phi_{\alpha_{N-1}} \circ \cdots \circ \Phi_{\alpha_{0}}$. If $B$ is closed, the second term of the LeraySerre spectral sequence ${ }_{\mathrm{LS}} E_{r}^{p, q} \Rightarrow H^{p+q}(E, \partial E)$ is

$$
\mathrm{LS}_{2} E_{2}^{p, q} \simeq H^{p}\left(B ; \mathcal{H}^{q}(F, \partial F)\right) .
$$

We define now Morse cohomology of a closed manifold B with coefficients in a local system $\mathcal{S}$. Let $f: B \longrightarrow \mathbb{R}$ be a Morse function and $Y$ be a Morse-Smale negative pseudo-gradient vector field. Pick an orientation of 
the unstable manifolds of $Y$ and define the cohomological Morse complex with values in $\mathcal{S}$ as

$$
C^{k}(B ; Y, \mathcal{S})=\bigoplus_{\substack{x \in \operatorname{Crit}(f) \\ \operatorname{ind}_{\text {Morse }}(x)=k}} M_{x},
$$

with differential $\partial: C^{k} \longrightarrow C^{k+1}$ given by

$$
\partial(m\langle x\rangle)=\sum_{\operatorname{ind}_{\text {Morse }}(y)=k+1}\left(\sum_{\gamma \in \mathcal{M}(y, x)} n_{\gamma} \Phi_{\gamma}^{-1}(m)\right)\langle y\rangle .
$$

Here $x \in \operatorname{Crit}(f), m \in M_{x}$, $\operatorname{ind}_{\text {Morse }}(x)=k$ and $n_{\gamma}$ is the sign which is associated to the trajectory $\gamma$. The fundamental identity $\partial^{2}=0$ is proved by the usual gluing argument, taking into account that the cancelling pairs of trajectories form the boundary of a two-disc and therefore parallel transport is the same along the two "half-circles" forming its boundary. Similarly, one shows by a continuation argument that the resulting cohomology groups, denoted by $H^{*}(B ; Y, \mathcal{S})$, do not depend on the choice of Morse function, nor on the choice of pseudo-gradient vector field.

Any proof showing that Morse cohomology with constant coefficients is isomorphic to singular cohomology carries over to the case of locally constant coefficients. The approach that is most convenient for us is also the most geometric and uses cellular cohomology $H_{\text {Cell }}^{*}(B ; \mathcal{S})$ as an intermediate device. Given a CW-decomposition of $B$, let $B^{k}$ be the $k$-skeleton and define the cellular complex by $\operatorname{Cell}^{k}(B ; \mathcal{S})=H^{k}\left(B^{k}, B^{k-1} ; \mathcal{S}\right)$ with differential

$$
\partial_{\text {Cell }}: H^{k}\left(B^{k}, B^{k-1} ; \mathcal{S}\right) \rightarrow H^{k+1}\left(B^{k+1}, B^{k} ; \mathcal{S}\right)
$$

given by the connecting homomorphism in the long exact sequence of the triple $\left(B^{k+1}, B^{k}, B^{k-1}\right)$. It is shown in $[\mathbf{2 4}$, Appendix A.4] that we have a canonical isomorphism $H^{*}\left(\operatorname{Cell}^{*}(B ; \mathcal{S}), \partial_{\text {Cell }}\right) \simeq H^{*}(B ; \mathcal{S})$ if $\mathcal{S}$ is constant, but the proof carries over verbatim to an arbitrary local system.

The connection with Morse homology is realized by expressing the cellular differential in an alternative way, using the incidence numbers of the cells $e_{i}^{k}$ of the CW-decomposition. Let $x_{i}^{k}$ be the center of the cell $e_{i}^{k}$. Each choice of orientation of the cells determines an isomorphism $\operatorname{Cell}^{k}(B ; \mathcal{S}) \simeq \bigoplus_{i} M_{x_{i}^{k}}$ and incidence numbers $\left[e_{i}^{k}: e_{j}^{k+1}\right]$. The differential $\partial_{\text {Cell }}$ is then equal to

$$
\partial_{\mathrm{Cell}}\left(m_{i}\right)=\sum_{j}\left[e_{i}^{k}: e_{j}^{k+1}\right] \Phi_{i j}\left(m_{i}\right), \quad m_{i} \in M_{x_{i}^{k}},
$$

where $\Phi_{i j}$ is parallel transport along a path from $x_{i}^{k}$ to $x_{j}^{k+1}$ contained in the closure of $e_{j}^{k+1}$. In order for parallel transport to be independent of the path, it is enough that the closure of $e_{j}^{k+1}$ be simply-connected. This can fail only if $k=0$ and the endpoints of $e_{j}^{1}$ coincide with some $e_{i}^{0}$, in which 
case the term $\left[e_{i}^{0}: e_{j}^{1}\right] \Phi_{i j}\left(m_{i}\right)$ has to be replaced by $\left\langle e_{i}^{0}\right\rangle\left(\Phi_{i j}^{+}\left(m_{i}\right)-\Phi_{i j}^{-}\left(m_{i}\right)\right)$. Here $\Phi_{i j}^{ \pm}$is the path running from $x_{i}^{0}$ to $x_{j}^{1}$ and having the same/the opposite orientation as $e_{j}^{1}$, and $\left\langle e_{i}^{0}\right\rangle$ is the sign (orientation) of $e_{i}^{0}$. We refer to $[\mathbf{3}$, IV.10] for a proof of (7.2) in the case where $\mathcal{S}$ is constant, which carries over verbatim to the case of an arbitrary local system.

We now use the fact proved by Laudenbach [20] that if the vector field $Y$ is equal near its zeroes to the negative gradient of a quadratic form with respect to the Euclidean metric, then its unstable manifolds provide a CWdecomposition of $B$. It then follows directly from the definitions that

$$
\left[W^{u}(x): W^{u}(y)\right]=\sum_{\gamma \in \mathcal{M}(y, x)} n_{\gamma} .
$$

In particular, once an orientation of the unstable manifolds has been chosen, the Morse complex $\left(C^{*}(B ; Y, \mathcal{S}), \partial\right)$ is canonically identified with the cellular complex $\left(\operatorname{Cell}^{*}(B ; \mathcal{S}), \partial_{\text {Cell }}\right)$, and we have canonical isomorphisms

$$
H^{*}(B ; Y, \mathcal{S}) \simeq H_{\text {Cell }}^{*}(B ; \mathcal{S}) \simeq H^{*}(B ; \mathcal{S}) .
$$

7.2.2. Local subsystems and extensions. The motivation for introducing local subsystems is that parallel transport is defined in the Floer setting only along certain paths in $B$. The question arises whether such a system of isomorphisms can be extended to a local system, and if yes, in how many non-isomorphic ways. The notion of a local subsystem is a convenient way to organize the available data.

Definition 7.4. Let $R$ be a ring and $M$ an $R$-module. A local subsystem with fiber $M$ on the topological space $B$ consists of the following data.

1) a subset $C \subset B$ and one copy $M_{x}$ of $M$ for each $x \in C$;

2) a subset $\mathcal{P} \subset \mathcal{P}(B)$ such that

- if $\alpha \in \mathcal{P}$, then $\alpha(0), \alpha(1) \in C$;

- for any $x \in C$, the constant path $\underline{x}(\cdot) \equiv x$ belongs to $\mathcal{P}$;

- $\alpha \in \mathcal{P}$ if and only if $\alpha^{-1} \in \mathcal{P}$;

- if $\alpha, \beta \in \mathcal{P}$ and $\alpha(1)=\beta(0)$, then $\alpha \cdot \beta \in \mathcal{P}$;

3) a family of isomorphisms $\Phi=\left(\Phi_{\alpha}: M_{\alpha(0)} \stackrel{\sim}{\longrightarrow} M_{\alpha(1)}\right)_{\alpha \in \mathcal{P}}$ such that

- if $\alpha \simeq \beta$ are homotopic in $B$ with fixed endpoints, then $\Phi_{\alpha}=\Phi_{\beta}$;

- if $\alpha, \beta \in \mathcal{P}$ and $\alpha(1)=\beta(0)$, then $\Phi_{\alpha \cdot \beta}=\Phi_{\beta} \circ \Phi_{\alpha}$.

Definition 7.5. We call the pair $\operatorname{Supp}(\mathcal{S})=(C, \mathcal{P})$ the support of the local subsystem $\mathcal{S}=(C, \mathcal{P}, \Phi)$.

The connected component $\mathcal{S}\left(x_{0}\right)=\left(C\left(x_{0}\right), \mathcal{P}\left(x_{0}\right), \Phi\left(x_{0}\right)\right)$ of $x_{0}$ in $\mathcal{S}$ is defined as

$$
\begin{aligned}
& C\left(x_{0}\right)=\left\{x \in C: \exists \alpha \in \mathcal{P}, \alpha(0)=x_{0}, \alpha(1)=x\right\}, \\
& \mathcal{P}\left(x_{0}\right)=\left\{\alpha \in \mathcal{P}: \alpha(0), \alpha(1) \in C\left(x_{0}\right)\right\}, \\
& \Phi\left(x_{0}\right)=\left\{\Phi_{\alpha}: \alpha \in \mathcal{P}\left(x_{0}\right)\right\} .
\end{aligned}
$$


We say that $\mathcal{S}$ has connected support if $C\left(x_{0}\right)=C$ for some (and hence for any) $x_{0} \in C$.

The fundamental group $\pi_{1}\left(\mathcal{S}, x_{0}\right)$ of $\mathcal{S}$ at $x_{0}$ is defined as the set of homotopy classes in $B$ relative to $x_{0}$ of based loops in $\mathcal{P}\left(x_{0}\right)$. Multiplication is given by the catenation of loops.

Given two local subsystems $\mathcal{S}=(C, \mathcal{P}, \Phi)$ and $\mathcal{S}^{\prime}=\left(C^{\prime}, \mathcal{P}^{\prime}, \Phi^{\prime}\right)$, we say that $\mathcal{S}^{\prime}$ is an extension of $\mathcal{S}$, and write $\mathcal{S} \prec \mathcal{S}^{\prime}$ if $C \subset C^{\prime}, \mathcal{P} \subset \mathcal{P}^{\prime}$ and $\Phi \subset \Phi^{\prime}$.

Every local subsystem gives rise to a representation

$$
\chi_{\mathcal{S}, x_{0}}: \pi_{1}\left(\mathcal{S}, x_{0}\right) \longrightarrow \operatorname{Aut}\left(M_{x_{0}}\right) .
$$

Given $\mathcal{S} \prec \mathcal{S}^{\prime}$, we have an obvious inclusion $\pi_{1}\left(\mathcal{S}, x_{0}\right) \hookrightarrow \pi_{1}\left(\mathcal{S}^{\prime}, x_{0}\right)$ which fits into the commutative diagram

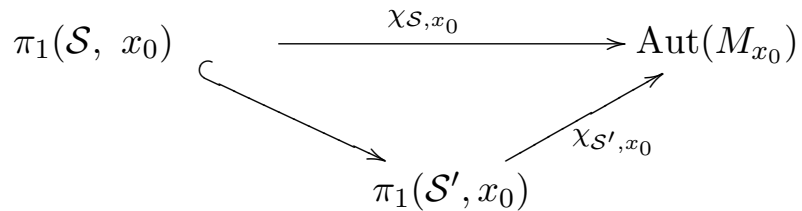

In view of the fact that local systems are in one-to-one correspondence with representations $\pi_{1}\left(B, x_{0}\right) \longrightarrow \operatorname{Aut}\left(M_{x_{0}}\right)$, the following statement is tautological.

Proposition 7.6. Let $\mathcal{S}$ be a local subsystem having connected support. Each extension of $\mathcal{S}$ to a local system corresponds to one and only one factorization

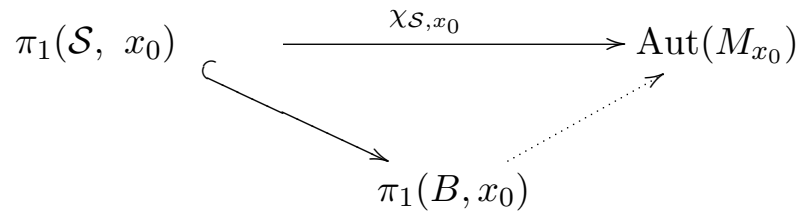

We see in particular that if a local subsystem $\mathcal{S}$ with connected support is such that the inclusion $\pi_{1}\left(\mathcal{S}, x_{0}\right) \hookrightarrow \pi_{1}\left(B, x_{0}\right)$ is an isomorphism, then $\mathcal{S}$ admits a unique extension to a local system.

7.3. Filtered Floer complexes. We construct in this section filtered Floer complexes using appropriate Hamiltonians, pseudo-gradient vector fields and almost complex structures.

Let $f: B \longrightarrow \mathbb{R}$ be a $C^{2}$-small Morse function, let $J_{B}$ be a timeindependent almost complex structure on $B$ and assume that the gradient $\nabla^{J_{B}} f$ is Morse-Smale and is equal to the gradient of a quadratic form with respect to the Euclidean metric near $\operatorname{Crit}(f)$. This last condition ensures by $[\mathbf{2 0}]$ that the unstable manifolds of $-\nabla^{J_{B}} f$ define a CW-decomposition 
of $B$. By Theorem 6.1, there exists $\tau>0$ such that all solutions of Floer's equation

$$
u_{s}+J_{B} u_{t}=J_{B} X_{f}
$$

with period less than $\tau$ are time-independent and cut the defining equation transversally. We note the fact that upon multiplying the function $f$ by a constant $c>0$, the corresponding $\tau$ transforms as $\tau \mapsto \tau / c$. As the function $f$ gets multiplied in the sequel by constants $0<c<1$, the bound $\tau>0$ can be taken uniform with respect to $c$.

The constructions in Sections 5 and 6 provide a bound $\epsilon_{0}>0$, sequences $0<\epsilon_{\nu} \leq \epsilon_{0}$ and $c_{\nu}>0, \nu \in \mathbb{N}$, as well as sequences of $\tau$-periodic Hamiltonians $K_{\nu}$, split almost complex structures $J_{\nu}$ and vector fields $Y_{\nu}^{\epsilon}, 0<\epsilon \leq \epsilon_{0}$, such that

(A) The $\tau$-periodic orbits of $\epsilon K_{\nu}, 0<\epsilon \leq \epsilon_{0}$ are nondegenerate and located in the fibers lying over the critical points of $f$;

(B) The constant $\tau$-periodic orbits of $\epsilon K_{\nu}$ are critical points of $K_{\nu}$, the vector field $Y_{\nu}^{\epsilon}, 0<\epsilon \leq \epsilon_{0}$, is a negative pseudo-gradient for $K_{\nu}$ on $E$ and $Y_{\nu}^{\epsilon}$ is equal to the gradient of a quadratic form with respect to the Euclidean metric near $\operatorname{Crit}\left(K_{\nu}\right)$;

(C) The vector field

$$
\mathcal{Y}_{\nu}^{\epsilon}(x)=J_{\nu} \dot{x}-Y_{\nu}^{\epsilon} \circ x
$$

defined on contractible $\tau$-periodic loops in $\widehat{E}$ is a strong pseudogradient for the action functional $A_{\epsilon K_{\nu}}^{\epsilon}$ for $0<\epsilon \leq \epsilon_{0}$;

(D) For $0<\epsilon \leq \epsilon_{\nu}$, the $\tau$-periodic solutions of Floer's equation

$$
u_{s}+J_{\nu} u_{t}=Y_{\nu}^{\epsilon}(t, u(s, t))
$$

cut the equation transversally. The resulting Floer complexes are denoted

$$
F C^{*}(\epsilon, \nu)=F C^{*}\left(Y_{\nu}^{\epsilon}, J_{\nu}\right)
$$

(E) Solutions of (7.3) project on gradient trajectories of $\epsilon c_{\nu} f$. We denote

$$
C^{*}(\epsilon, \nu)=C^{*}\left(B ;-\epsilon c_{\nu} \nabla^{J_{B}} f\right) ;
$$

(F) The Hamiltonians $K_{\nu}$ form a cofinal sequence.

Statements (A) and (C) follow from Proposition 5.8. Property (B) is realized by choosing the perturbations $f_{i}$ in Section 5.2 quadratic near their critical points. Statement (D) follows from Theorem 6.5 and by noticing that the perturbations of almost complex structures can be taken trivial near the critical points of $K_{\nu}$, so that property (B) is preserved. Statement (E) follows from the special form of the vector fields $Y_{\nu}^{\epsilon}$. Property (F) is realized by choosing a cofinal "stem sequence" $h_{\nu}$ in order to construct the Hamiltonians $K_{\nu}$. 
Remark 7.7 (Rescaling). Cofinality does not depend on the rescaling $\Omega \rightsquigarrow \epsilon \Omega$, because the coordinate $S$ depends only on the vertical vector field $Z$, which remains unchanged. On the other hand, the action of the orbits corresponding to closed characteristics gets multiplied by a factor $\epsilon$ under the rescaling $\Omega \rightsquigarrow \epsilon \Omega$.

Remark 7.8 (Parameters $\epsilon_{\nu} \rightarrow 0$ are unavoidable). The use of the constants $\epsilon_{\nu}$ cannot be avoided because of transversality issues, and more precisely because of Lemma 6.7. The constants $c_{\nu}$ have more of a formal role, mainly in order to ensure $K_{\nu} \leq K_{\nu^{\prime}}$ for $\nu \leq \nu^{\prime}$ (cf. Section 5.2).

Let $R$ be a ring and $-\infty \leq a<b \leq \infty$ such that $\tau a, \tau b \notin \operatorname{Spec}(\partial E)$. We define

$$
F_{p} C_{[\epsilon a, \epsilon b]}^{*}(\epsilon, \nu)=\bigoplus_{\substack{\alpha \in \mathcal{P}\left(K_{\nu}\right) \\ A_{\epsilon K_{\nu}}^{\epsilon}(\alpha) \in[\epsilon a, \epsilon b] \\-i_{\mathrm{CZ}}(\pi(\alpha)) \geq p}} R\langle\alpha\rangle \subseteq F C_{[\epsilon a, \epsilon b]}^{*}(\epsilon, \nu) .
$$

Here $\mathcal{P}\left(K_{\nu}\right)$ denotes the set of $\tau$-periodic orbits of $K_{\nu}$.

Proposition 7.9. Formula (7.4) defines a filtration, i.e.,

$$
\partial\left(F_{p} C_{[\epsilon a, \epsilon b]}^{k}(\epsilon, \nu)\right) \subset F_{p} C_{[\epsilon a, \epsilon b]}^{k+1}(\epsilon, \nu) .
$$

Proof. For $\alpha \in \mathcal{P}\left(K_{\nu}\right)$ such that $A_{\epsilon K_{\nu}}^{\epsilon}(\alpha) \in[\epsilon a, \epsilon b]$ and $-i_{\mathrm{CZ}}(\pi(\alpha)) \geq p$, we have

$$
\partial\langle\alpha\rangle=\sum_{\substack{\beta:-i_{\mathrm{CZ}}(\beta)+i_{\mathrm{CZ}}(\alpha)=1 \\ A_{\epsilon K_{\nu}}^{\epsilon}(\beta) \in[\epsilon a, \epsilon b]}} \#(\mathcal{M}(\beta, \alpha) / \mathbb{R})\langle\beta\rangle .
$$

For each orbit $\beta$ appearing in the above sum, there exists a solution of Floer's equation $u_{s}+J_{\nu} u_{t}=Y_{\nu}^{\epsilon}(t, u(s, t))$ running from $\beta$ to $\alpha$. It follows that $v=\pi \circ u$ is a solution of the equation $v_{s}+J_{B} v_{t}=\epsilon c_{\nu} J_{B} X_{f}(v)$ running from $\pi(\beta)$ to $\pi(\alpha)$. By transversality, we must have $\operatorname{ind}_{\text {Morse }}(\pi(\beta) ; f) \geq$ $\operatorname{ind}_{\text {Morse }}(\pi(\alpha) ; f)$, with equality if and only if $v$ is constant i.e., $u$ is entirely contained in the critical fiber lying over the point $\pi(\beta)=\pi(\alpha)$. Equivalently, we get $-i_{\mathrm{CZ}}(\pi(\beta)) \geq-i_{\mathrm{CZ}}(\pi(\alpha)) \geq p$.

The filtration (7.4) defines a spectral sequence

$$
E_{r}^{p, q}(\epsilon, \nu, a, b)
$$

such that

$$
E_{r}^{p, q}(\epsilon, \nu, a, b) \Longrightarrow F H_{[\epsilon a, \epsilon b]}^{p+q}(\epsilon, \nu) .
$$


7.4. The terms $E_{1}$ and $E_{2}$. The filtered complex $F C_{[\epsilon a, \epsilon b]}^{*}(\epsilon, \nu)$ falls under the formalism of Example 7.1. The proof of Proposition 7.9 shows that the component $\partial_{0}$ of the differential is given by the Floer differential in the fibers, hence

$$
E_{1} \simeq \bigoplus_{p \in \mathbb{Z}} \bigoplus_{\substack{p_{i} \in \operatorname{Crit}(f) \\-i_{\mathrm{CZ}}\left(p_{i}\right)=p}} F H_{[\epsilon a, \epsilon b]}^{*}\left(\widehat{E}_{p_{i}},\left.J_{\nu}\right|_{\widehat{E}_{p_{i}}},\left.\epsilon K_{\nu}\right|_{\widehat{E}_{p_{i}}}, \epsilon \Omega\right),
$$

and $d_{1}: E_{1} \longrightarrow E_{1}$ acts by

$$
\begin{aligned}
& \underbrace{[\alpha]} \longmapsto[\quad \#(\mathcal{M}(\beta, \alpha) / \mathbb{R})\langle\beta\rangle] . \\
& -i_{\mathrm{CZ}}(\alpha)=k \quad-i_{\mathrm{CZ}}(\beta)=k+1 \\
& -i_{\mathrm{CZ}}(\pi(\alpha))=p \quad-i_{\mathrm{CZ}}(\pi(\beta))=p+1
\end{aligned}
$$

Here the representative $\alpha$ of the cohomology class is an $R$-linear combination of periodic orbits $\alpha_{i}$ with $-i_{\mathrm{CZ}}\left(\alpha_{i}\right)=k$ and lying in the same fiber over a critical point of index $p$, while $\#(\mathcal{M}(\beta, \alpha) / \mathbb{R})$ represents, for some $\beta$ with $-i_{\mathrm{CZ}}(\beta)=k+1$, the extension by linearity in the second argument of the quantity $\#\left(\mathcal{M}\left(\beta, \alpha_{i}\right) / \mathbb{R}\right)$.

The formula for $d_{1}$ becomes transparent if we define the parallel transport map

$$
\begin{gathered}
\Phi_{\gamma}^{p_{j}, p_{i}}(\epsilon, \nu): F C^{q}\left(\widehat{E}_{p_{i}}, \epsilon, \nu\right) \longrightarrow F C^{q}\left(\widehat{E}_{p_{j}}, \epsilon, \nu\right), \\
\langle\alpha\rangle \longmapsto \sum_{-i_{\mathrm{CZ}}^{\mathrm{fiber}}(\beta)=q} \# \mathcal{M}_{\gamma}^{\epsilon, \nu}\left(p_{j} \otimes \beta, p_{i} \otimes \alpha\right)\langle\beta\rangle,
\end{gathered}
$$

where $p_{i}, p_{j} \in \operatorname{Crit}(f)$ with $-i_{\mathrm{CZ}}\left(p_{j}\right)>-i_{\mathrm{CZ}}\left(p_{i}\right), \gamma \in \mathcal{M}\left(p_{j}, p_{i}\right)$, $F C^{q}\left(\widehat{E}_{p_{i}}, \epsilon, \nu\right)$ and $F C^{q}\left(\widehat{E}_{p_{j}}, \epsilon, \nu\right)$ are the Floer complexes in the fibers $\widehat{E}_{p_{i}}$, $\widehat{E}_{p_{j}}$ for the restrictions of $J_{\nu}, \epsilon K_{\nu}$ and $\epsilon \Omega$. We have denoted

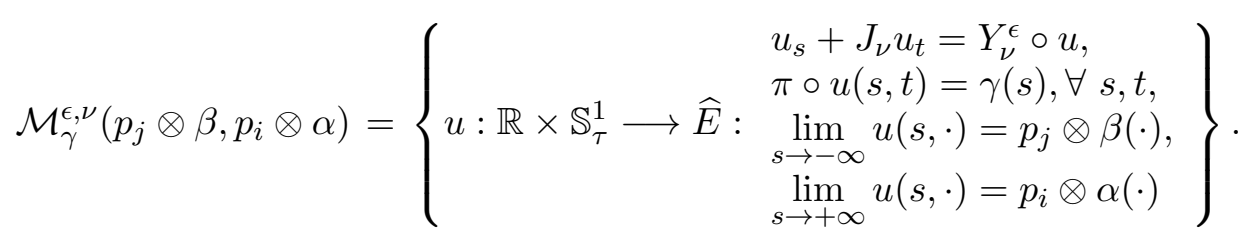

The notation $p_{i} \otimes \alpha$ stands for the orbit $\alpha$ in the fiber $\widehat{E}_{p_{i}}$ viewed as an orbit in $\widehat{E}$, and $p_{j} \otimes \beta$ has a similar meaning. It is easy to see that we have

$$
\operatorname{dim} \mathcal{M}_{\gamma}^{\epsilon, \nu}\left(p_{j} \otimes \beta, p_{i} \otimes \alpha\right)=-i_{\mathrm{CZ}}^{\mathrm{fiber}}(\beta)+i_{\mathrm{CZ}}^{\mathrm{fiber}}(\alpha)
$$

regardless of the difference of indices between $p_{j}$ and $p_{i}$.

We prove in Lemma 7.10 below that $\Phi_{\gamma}^{p_{j}, p_{i}}(\epsilon, \nu)$ is a morphism of differential complexes which induces an isomorphism in homology and preserves 
the action filtration. As a consequence, the differential $d_{1}$ can be rewritten

$$
\begin{aligned}
& d_{1}(\epsilon, \nu): \bigoplus_{p_{i} \in \operatorname{Crit}(f)} F H_{[\epsilon a, \epsilon b]}^{*}\left(\widehat{E}_{p_{i}}, \epsilon, \nu\right) \longrightarrow \bigoplus_{p_{i} \in \operatorname{Crit}(f)} F H_{[\epsilon a, \epsilon b]}^{*}\left(\widehat{E}_{p_{i}}, \epsilon, \nu\right), \\
& p_{i} \otimes[\alpha] \longmapsto p_{-i_{\mathrm{CZ}}\left(p_{j}\right)=-i_{\mathrm{CZ}}\left(p_{i}\right)+1} \otimes \sum_{[\gamma] \in \mathcal{M}\left(p_{j}, p_{i}\right) / \mathbb{R}} n_{\gamma} \Phi_{\gamma}^{p_{j}, p_{i}}(\epsilon, \nu) \cdot[\alpha] .
\end{aligned}
$$

Here $\alpha \in F C_{[\epsilon a, \epsilon b]}^{*}\left(\widehat{E}_{p_{i}}, \epsilon, \nu\right)$ is such that $\partial_{\text {fiber }}(\alpha)=0$. We shall moreover prove in Proposition 7.12 below that there is a unique local system $\mathcal{F H}_{[\epsilon a, \epsilon b]}^{q}(\widehat{F}, \epsilon, \nu)$ on $B$ with fiber $F H_{[\epsilon a, \epsilon b]}^{q}(\widehat{F}, \epsilon, \nu)$ so that the maps $\Phi_{\gamma}^{p_{j}, p_{i}}$ described above are the parallel transport maps with respect to this local system. It then follows from the definition (7.1) of Morse cohomology with values in a local system that

$$
E_{2}^{p, q}(\epsilon, \nu, a, b) \simeq H\left(E_{1}, d_{1}\right)=H^{p}\left(B ; \mathcal{F} \mathcal{H}_{[\epsilon a, \epsilon b]}^{q}(\widehat{F}, \epsilon, \nu)\right) .
$$

7.5. The Floer local system. We prove in this section that the parallel transport maps $\Phi_{\gamma}^{p_{j}, p_{i}}(\epsilon, \nu)$ are chain morphisms which induce isomorphisms in homology, and moreover they can be incorporated into a uniquely determined local system on $B$.

Lemma 7.10. The map $\Phi_{\gamma}^{p_{j}, p_{i}}(\epsilon, \nu)$ in (7.7) is a morphism of differential complexes:

$$
\Phi_{\gamma}^{p_{j}, p_{i}} \circ \partial_{p_{i}}+\partial_{p_{j}} \circ \Phi_{\gamma}^{p_{j}, p_{i}}=0,
$$

where $\partial_{p_{i}}, \partial_{p_{j}}$, are the Floer differentials in the fibers $\widehat{E}_{p_{i}}, \widehat{E}_{p_{j}}$, respectively. Moreover, it induces an isomorphism in Floer cohomology and preserves the action filtration on Floer complexes.

Proof. The idea is to identify the moduli spaces $\mathcal{M}_{\gamma}^{\epsilon, \nu}\left(p_{j} \otimes \beta, p_{i} \otimes \alpha\right)$ with the moduli spaces $\mathcal{M}(\beta, \alpha)$ corresponding to a deformation of the Floer equations on the fibers $\widehat{E}_{p_{i}}, \widehat{E}_{p_{j}}$. More precisely, let us choose a symplectic trivialization

$$
\begin{gathered}
\Psi:\left.\widehat{E}\right|_{\operatorname{im} \gamma} \longrightarrow \mathbb{R} \times \widehat{F} \\
\widehat{E}_{\gamma(s)} \longrightarrow\{s\} \times \widehat{F}
\end{gathered}
$$

which, under the projection $\gamma(s) \longmapsto s$ and after having chosen isomorphisms $\widehat{F} \simeq \widehat{E}_{p_{i}} \simeq \widehat{E}_{p_{j}}$, coincides with the trivializations $\Psi_{i}, \Psi_{j}$ of Section 5.2 on $\operatorname{im} \gamma \cap U_{i}$, respectively, on $\operatorname{im} \gamma \cap U_{j}$. In this trivialization, we can interpret

$$
\widehat{K}=K_{\nu} \circ \Psi^{-1}
$$

as an $s$-dependent deformation from $K_{\nu} \circ \Psi_{p_{i}}^{-1}=\varphi+\chi_{\nu}^{\delta}+c_{-}$to $K_{\nu} \circ$ $\Psi_{p_{j}}^{-1}=\varphi+\chi_{\nu}^{\delta}+c_{+}$, where $\chi_{\nu}^{\delta}$ is the perturbation described in Section 5.2 and $c_{ \pm}$are arbitrarily small constants. Writing $\widehat{K}=\left(\widehat{K}_{s}\right), s \in \mathbb{R}$, with 
$\widehat{K}_{s}: \mathbb{S}_{\tau}^{1} \times \widehat{F} \longrightarrow \mathbb{R}$ and considering on $\{s\} \times \widehat{F}$ the almost complex structure $J_{s}^{\mathrm{vert}}$ induced from $\widehat{E}_{\gamma(s)}$, the vector field $Y_{\nu}^{\epsilon}$ takes the form

$$
\widehat{Y}_{\nu}^{\epsilon}(s, p)=\nabla_{s}^{J_{s}^{\mathrm{vert}}} \epsilon \widehat{K}_{s}+\frac{\partial}{\partial s},
$$

with $\nabla_{s}^{J_{s}^{\text {ert }}}$ the Levi-Civita connection for $\epsilon \Omega$. We infer that, if $\alpha$ and $\beta$ are two periodic orbits of $\varphi+\chi_{\nu}^{\delta}$, the moduli space $\mathcal{M}_{\gamma}^{\epsilon, \nu}\left(p_{j} \otimes \beta, p_{i} \otimes \alpha\right)$ is isomorphic to the moduli space

$$
\mathcal{M}(\beta, \alpha ; \widehat{K})=\left\{u: \mathbb{R} \times \mathbb{S}_{\tau}^{1} \longrightarrow \widehat{F}: \begin{array}{l}
u_{s}+J_{s}^{\text {vert }} u_{t}=\nabla^{J_{s}^{\text {vert }}} \epsilon \widehat{K}_{s} \\
u(-\infty)=\beta, u(+\infty)=\alpha
\end{array}\right\}
$$

It is a standard fact in the Floer theory that the count of the elements of these moduli spaces gives rise to chain morphisms which induce isomorphisms in homology. Moreover, parallel transport preserves the filtration by the action if the constants $c_{ \pm}$are chosen small enough.

In the next lemma, we omit the indices $\epsilon$ and $\nu$ to improve readability.

Lemma 7.11. Let $x, y, z \in \operatorname{Crit}(f)$ and $u \in \mathcal{M}(x, y), v \in \mathcal{M}(y, z)$. Let $\gamma \in \mathcal{M}(x, z)$ be such that $[\gamma] \in \mathcal{M}(x, z) / \mathbb{R}$ belongs to a component whose boundary contains $([u],[v]) \in(\mathcal{M}(x, y) / \mathbb{R}) \times(\mathcal{M}(y, z) / \mathbb{R})$. The following equality holds:

$$
\Phi_{\gamma}^{x, z}=\Phi_{u}^{x, y} \circ \Phi_{v}^{y, z}
$$

Proof. We first remark that $\Phi_{\gamma}^{x, z}$ is independent of $\gamma$ as long as $[\gamma]$ varies in one component of $\mathcal{M}(x, z) / \mathbb{R}$. Indeed, components are path connected and we can choose an embedded path $\left[\gamma_{\tau}\right], \tau \in[0,1]$ between any two given points $\left[\gamma_{0}\right]$ and $\left[\gamma_{1}\right]$. A choice of symplectic trivialization of $\widehat{E}$ over $\bigcup_{\tau} \operatorname{im} \gamma_{\tau}$ induces a homotopy between the homotopies $\left(\widehat{K}_{0}, \widehat{Y}_{0}\right)$ and $\left(\widehat{K}_{1}, \widehat{Y}_{1}\right)$ corresponding to $\gamma_{0}$ and $\gamma_{1}$ through Lemma 7.10. The corresponding homomorphisms are then chain homotopic and coincide at the level of homology.

It is therefore enough to prove the claim if $[\gamma]$ lies in the image of the gluing map

$$
\#: K \times\left[R_{0}, \infty[\longrightarrow \mathcal{M}(x, z) / \mathbb{R},\right.
$$

where $K$ is a relatively compact open neighborhood of the point $([u],[v])$ in $(\mathcal{M}(x, y) / \mathbb{R}) \times(\mathcal{M}(y, z) / \mathbb{R})$ and $R_{0}>0$ is large enough. The key point, borrowed from the proof of the invariance of Floer homology [26, Lemma 3.11], is that the morphism $\Phi_{u}^{x, y} \circ \Phi_{v}^{y, z}$ is induced by the gluing of the two homotopies $\left(\widehat{K}_{0}, \widehat{Y}_{0}\right),\left(\widehat{K}_{1}, \widehat{Y}_{1}\right)$ into

$$
\left(\widehat{K}_{01}, \widehat{Y}_{01}\right):= \begin{cases}\left(\widehat{K}_{0}, \widehat{Y}_{0}\right)(s+R), & s \leq 1 \\ \left(\widehat{K}_{1}, \widehat{Y}_{1}\right)(s-R), & s \geq-1\end{cases}
$$


Here $R$ is chosen large enough so that the resulting homotopy is regular. Ignoring the additive constants as in Lemma 7.10, this homotopy is a deformation of the constant Hamiltonian $\varphi+\chi_{\nu}^{\delta}$.

For $R$ large enough, the image of $[\gamma]=\#(([u],[v]), R)$ lies in a contractible neighborhood $\mathcal{U}$ of im $u \cup \operatorname{im} v$ and we choose a symplectic trivialization of $\widehat{E}$ over $\mathcal{U}$ which extends the trivialization over $\operatorname{im} u \cup \operatorname{im} v$ which was implicit in the construction of $\left(\widehat{K}_{01}, \widehat{Y}_{01}\right)$. The morphism $\Phi_{\gamma}^{x, z}$ now arises from a homotopy $\left(\widehat{K}_{\gamma}, \widehat{Y}_{\gamma}\right)$ which, in the given trivialization, is also a deformation of the constant Hamiltonian $\varphi+\chi_{\nu}^{\delta}$.

As a conclusion, both homotopies induce the identity in the given trivialization, and in particular $\Phi_{\gamma}^{x, z}=\Phi_{u}^{x, y} \circ \Phi_{v}^{y, z}$.

In order to incorporate the maps $\Phi_{\gamma}^{p_{j}, p_{i}}(\epsilon, \nu)$ in local systems for the various values of $q \in \mathbb{Z}$, we exhibit local subsystems $\left(C, \mathcal{P}, \Phi^{q}\right)$ (cf. Section 7.2.2) determined by these maps. We recall that we denote by $\mathcal{P}(B)$ the space of continuous paths in $B$. We define

$$
C=\operatorname{Crit}(f), \quad \mathcal{P}=\langle\text { negative gradient trajectories of } f\rangle_{\operatorname{Crit}(f)},
$$

where, for $\mathcal{R} \subset \mathcal{P}(B)$ and $C \subset B$, the notation $\langle\mathcal{R}\rangle_{C}$ stands for the minimal subset of $\mathcal{P}(B)$ which contains $\mathcal{P}$ and which satisfies condition (2) of Definition 7.4. In our case, $\mathcal{P}$ consists of chains of negative gradient trajectories of $f$ and their inverses. We define

$$
\Phi^{q}=\left(\Phi_{\alpha}: F H_{[\epsilon a, \epsilon b]}^{q}\left(\widehat{E}_{\alpha(0)}, \epsilon, \nu\right) \longrightarrow F H_{[\epsilon a, \epsilon b]}^{q}\left(\widehat{E}_{\alpha(1)}, \epsilon, \nu\right)\right)_{\alpha \in \mathcal{P}}
$$

on the generators of $\mathcal{P}$ by

$$
\Phi_{\gamma}=\Phi_{\gamma}^{\gamma(-\infty), \gamma(+\infty)}(\epsilon, \nu), \quad \gamma \text { negative gradient trajectory of } f .
$$

The next result is of a topological nature, although its statement involves Floer homology groups. The transition to Floer homology is realized through a repeated use of Lemma 7.11.

Proposition 7.12. a) For any $q \in \mathbb{Z}$ and $-\infty \leq a<b \leq \infty$, the triple $\left(C, \mathcal{P}, \Phi^{q}\right)$ defines a local subsystem with fiber $F H_{[\epsilon a, \epsilon b]}^{q}(\widehat{F}, \epsilon, \nu)$, denoted by

$$
\underline{\mathcal{F H}}_{[\epsilon a, \epsilon b]}^{q}(\widehat{F}, \epsilon, \nu)
$$

b) If $B$ is connected, the support of the above local system is connected;

c) For any $p_{0} \in \operatorname{Crit}(f)$, the canonical inclusion

$$
\pi_{1}\left(\underline{\mathcal{F H}}_{[\epsilon a, \epsilon b]}^{q}(\widehat{F}, \epsilon, \nu), p_{0}\right) \hookrightarrow \pi_{1}\left(B, p_{0}\right)
$$

is an isomorphism.

Remark 7.13 (Truncated Floer local system). Assertion (c) implies together with Proposition 7.6 that, for each $q \in \mathbb{Z}$, there is a unique local 
system on $B$ extending $\mathcal{F \mathcal { H }}_{[\epsilon a, \epsilon b]}^{q}(\widehat{F}, \epsilon, \nu)$. We call it the truncated Floer local system and denote it by

$$
\mathcal{F} \mathcal{H}_{[\epsilon a, \epsilon b]}^{q}(\widehat{F}, \epsilon, \nu) \text {. }
$$

Proof of Proposition 7.12. Our proof crucially uses Lemma 7.11, as well as the assumption that the unstable manifolds of $-\nabla^{J_{B}} f$ provide a $\mathrm{CW}$ decomposition of $B$ (cf. Section 7.3). We denote the $k$-skeleton by

$$
B^{k}=\bigcup_{\operatorname{ind}_{\text {Morse }}(x) \leq k} W^{u}(x) .
$$

(a) We have to prove that parallel transport along a loop $\alpha \in \mathcal{P}$ which is null-homotopic in $B$ is trivial. Let $\alpha=\gamma_{0} \gamma_{1} \cdots \gamma_{N}$, where $\gamma_{i}$ is a trajectory of $\pm \nabla f$ running from $p_{i}$ to $p_{i+1}$ and $p_{0}=p_{N+1}$. By adding a chain $\eta_{0} \cdots \eta_{\ell} \eta_{\ell}^{-1} \cdots \eta_{0}$ running from $p_{0}$ to a local minimum of $f$, we can assume that ind Morse $\left(p_{0}\right)=0$. We are interested in the monodromy along $\alpha$ as an automorphism of $F H^{*}\left(\widehat{E}_{p_{0}}\right)$. By successively applying Lemma 7.11 and deforming the $\gamma_{i}$ 's to the boundary of $\mathcal{M}\left(p_{i}, p_{j}\right)$, we can assume that all trajectories $\gamma_{i}$ are of index 1 . We can moreover cancel pairs $\gamma_{i} \gamma_{i+1}, i \neq 0, N$, which are of the form $\eta \eta^{-1}$.

Claim. There exists a null-homotopic chain $\alpha_{1}$ based at $p_{0}$ which consists of trajectories connecting critical points of index 0 and 1 and which satisfies $\Phi_{\alpha_{1}}=\Phi_{\alpha}$.

The proof goes by induction over $m(\alpha)=\max _{i} \operatorname{ind}_{\text {Morse }}\left(p_{i}\right)$. Assume $m(\alpha) \geq 2$. We prove the existence of a null-homotopic chain $\alpha^{\prime}$ based at $p_{0}$ such that $m\left(\alpha^{\prime}\right)=m(\alpha)-1$ and $\Phi_{\alpha^{\prime}}=\Phi_{\alpha}$. Let us choose $p_{i}$ such that ind $\mathrm{Morse}_{i}\left(p_{i}\right)=m(\alpha)$. We claim that the pair $\gamma_{i-1} \gamma_{i}$ connecting $p_{i-1}$, $p_{i}$ and $p_{i+1}$ can be replaced without affecting parallel transport by a chain connecting $p_{i-1}$ and $p_{i+1}$ with intermediate critical points of index at most $m(\alpha)-1$. Moreover, this chain is homotopic to $\gamma_{i-1} \gamma_{i}$ with fixed endpoints, hence the resulting loop is still null-homotopic. Let us choose a path $\gamma$ : $[0,1] \longrightarrow W^{u}\left(p_{i}\right)$ with $\gamma(0) \in \operatorname{im} \gamma_{i-1}, \gamma(1) \in \operatorname{im} \gamma_{i}$ and which is transverse in $W^{u}\left(p_{i}\right)$ to the manifolds $W^{u}\left(p_{i}\right) \cap W^{s}(q), q \in \operatorname{Crit}(f)$. In particular, there exist points $0=t_{0}<t_{1}<\cdots<t_{\ell-1}<t_{\ell}=1$ such that the trajectory $\left[p_{i}, \gamma\left(t_{j}\right)\left[, 1 \leq j \leq \ell-1\right.\right.$, lands on a critical point $q_{j}$ of index 1 and, for every $t \in] t_{j-1}, t_{j}\left[, 1 \leq j \leq \ell\right.$, the trajectory $\left[p_{i}, \gamma(t)\right.$ [ lands on a critical point $m_{j}$ of index 0 (see Figure 1(A)). The boundaries of the moduli spaces $\mathcal{M}(x, y) / \mathbb{R}$ consist of broken trajectories and, together with the existence of the curve $\gamma$, this ensures that the trajectories $\gamma_{i-1},\left[p_{i}, q_{j}\right], 1 \leq j \leq \ell-1$, and $\gamma_{i}$ can be completed to broken chains which lie pairwise in the boundary of connected components of spaces $\mathcal{M}\left(p_{i}, m\right) / \mathbb{R}$, with $m$ a critical point of index 0 . More precisely, there exist index decreasing chains of index 1 trajectories from $p_{i-1}$ to $m_{1}$ and from $p_{i+1}$ to $m_{\ell}$, denoted $\beta_{i-1}$ and $\beta_{i}$, as well as trajectories $\left[m_{j}, q_{j}\right],\left[q_{j}, m_{j+1}\right], 1 \leq j \leq \ell-1$, such that the 
pairs $\left(q_{i-1}^{-1} \beta_{i-1},\left[p_{i}, q_{1}\right] \cdot\left[q_{1}, m_{1}\right]\right),\left(\left[p_{i}, q_{j}\right] \cdot\left[q_{j}, m_{j+1}\right],\left[p_{i}, q_{j+1}\right] \cdot\left[q_{j+1}, m_{j+1}\right]\right)$, $1 \leq j \leq \ell-2$, and $\left(\left[p_{i}, q_{\ell-1}\right] \cdot\left[q_{\ell-1}, m_{\ell}\right], \gamma_{i} \cdot \beta_{i}\right)$ lie in the boundary of the same components of $\mathcal{M}\left(p_{i}, m_{j}\right) / \mathbb{R}, 1 \leq j \leq \ell$, respectively. The chain $\gamma_{i-1} \gamma_{i}$ is now to be replaced by

$$
\beta_{i-1} \cdot \prod_{1 \leq j \leq \ell-1}\left[m_{j}, q_{j}\right] \cdot\left[q_{j}, m_{j+1}\right] \cdot \beta_{i}^{-1} .
$$

The construction can be repeated until all points of index $m(\alpha)$ are eliminated.

Claim. We have $\Phi_{\alpha_{1}}=\mathrm{Id}$.

For any CW-decomposition $B=\bigcup_{k>0} B^{k}$, the group $\pi_{1}\left(B^{1}\right)$ is free, the group $\pi_{1}\left(B^{2}\right)$ is the quotient of $\pi_{1}\left(B^{1}\right)$ by the normal subgroup generated by the boundary cycles of the 2-cells and the map $\pi_{1}\left(B^{2}\right) \longrightarrow \pi_{1}(B)$ induced by the inclusion $B^{2} \hookrightarrow B$ is an isomorphism. In order to prove that parallel transport is trivial along the null-homotopic loop $\alpha_{1}$, we can therefore assume without loss of generality that $\alpha_{1}$ is the boundary cycle of a 2-cell $W^{u}(p), \operatorname{ind}_{\text {Morse }}(p)=2$, i.e., we can write

$$
\alpha_{1}=\prod_{j=0}^{N}\left[p_{j}, q_{j}\right] \cdot\left[q_{j}, p_{j+1}\right]
$$

with $\operatorname{ind}_{\text {Morse }}\left(p_{j}\right)=0, \operatorname{ind}_{\text {Morse }}\left(q_{j}\right)=1, p_{N+1}=p_{0}$. Each $q_{j}$ is understood to be equipped with a trajectory $\beta_{j}=\left[p, q_{j}\right]$ such that

$$
\bigcup_{j} \beta_{j}=\bigcup_{\operatorname{ind}_{\text {Morse }}(q)=1} W^{u}(p) \cap W^{s}(q) .
$$

The ordering of the $q_{j}$ 's is such that $\beta_{j}$ and $\beta_{j+1}, 0 \leq j \leq N$, lie in the boundary of one same component of $\mathcal{M}(p, m)$, $\operatorname{ind}_{\text {Morse }}(m)=0$ (see Figure $1(\mathrm{~B}))$.

A deformation argument based on Lemma 7.11 and entirely similar to that of Case 1 shows that parallel transport along $\alpha_{1}$ is the same as parallel transport along the loop

$$
\left[p_{0}, q_{0}\right] \cdot \prod_{j=0}^{N-1} \beta_{j}^{-1} \beta_{j+1} \cdot\left[q_{N}, p_{0}\right]=\left[p_{0}, q_{0}\right] \cdot \beta_{0}^{-1} \beta_{N} \cdot\left[q_{N}, p_{0}\right] .
$$

The last loop is the boundary of a component of $\mathcal{M}\left(p, p_{0}\right)$ and, again by Lemma 7.11, the induced parallel transport is trivial.

(b) Every critical point is connected by a trajectory to a point of index 0 . On the other hand, because $B$ is connected and the unstable manifolds form a CW-decomposition, the 1-skeleton has to be connected and any two index 0 points are therefore connected by a chain of index 1 trajectories. This shows that the support of the local system is connected. 




(A)

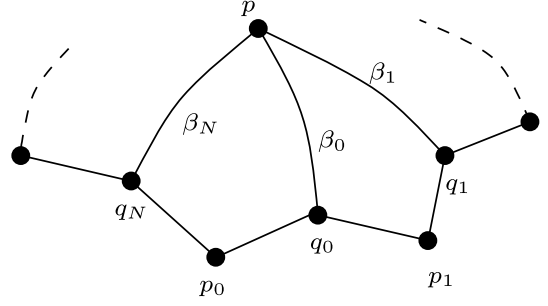

(B)

Figure 1. Parallel transport is trivial along null-homotopic loops.

(c) We have to prove surjectivity of the map under study. We use the fact that the map $\pi_{1}\left(B^{1}\right) \longrightarrow \pi_{1}(B)$ is surjective, which means that every homotopy class in $B$ has a representative which is supported in the 1-skeleton $B^{1}=\bigcup_{\text {ind }_{\text {Morse }}(p)=1} W^{u}(p)$. From this, it is easy to find a representative given by a chain of trajectories connecting points of index 0 and 1 .

7.6. Proof of the main theorems. We prove in this section Theorems $\mathrm{A}$ and $\mathrm{B}$ stated in the Introduction. We need four Lemmas which describe the behavior of the homology groups $F H_{[\epsilon a, \epsilon b]}^{*}\left(\epsilon K_{\nu}, \omega_{\epsilon}\right)$, of the complexes $F C_{[\epsilon a, \epsilon b]}^{*}\left(Y_{\nu}^{\epsilon}, J_{\nu}\right)$ and of the local systems $\mathcal{F H}_{[\epsilon a, \epsilon b]}^{q}(\widehat{F}, \epsilon \nu)$ as one of the parameters $\epsilon$ and $\nu$ varies and the other one is fixed. We choose $-\infty \leq a<b \leq \infty$ such that $\tau a, \tau b \notin \operatorname{Spec}(\partial E)$.

Lemma 7.14. Let $\nu \in \mathbb{N}$ be fixed. For any $0<\epsilon<\epsilon^{\prime} \leq \epsilon_{0}$, we have natural isomorphisms

$$
F H_{[\epsilon a, \epsilon b]}^{*}\left(\epsilon K_{\nu}, \omega_{\epsilon}\right) \stackrel{\psi_{\nu}^{\epsilon^{\prime}, \epsilon}}{\longrightarrow} F H_{\left[\epsilon^{\prime} a, \epsilon^{\prime} b\right]}^{*}\left(\epsilon^{\prime} K_{\nu}, \omega_{\epsilon^{\prime}}\right)
$$

which satisfy $\psi_{\nu}^{\epsilon^{\prime \prime}, \epsilon^{\prime}} \circ \psi_{\nu}^{\epsilon^{\prime}, \epsilon}=\psi_{\nu}^{\epsilon^{\epsilon^{\prime \prime}}, \epsilon}, \epsilon<\epsilon^{\prime}<\epsilon^{\prime \prime}$. The Floer complexes are based on $\tau$-periodic orbits and are understood to involve transverse almost complex structures.

Proof. The periodic orbits involved in the two complexes are the same. Let us consider a homotopy of Hamiltonians and symplectic forms $\left(\epsilon(s) K_{\nu}, \omega_{\epsilon(s)}\right)$, $s \in \mathbb{R}$, from $\left(\epsilon K_{\nu}, \omega_{\epsilon}\right)$ to $\left(\epsilon^{\prime} K_{\nu}, \omega_{\epsilon^{\prime}}\right)$. The periodic orbits involved in the Floer complexes remain geometrically the same during the homotopy, but their action gets multiplied by $\epsilon(s)$. As a consequence, the extremities of the action interval $[\epsilon(s) a, \epsilon(s) b]$ which interpolates between $[\epsilon a, \epsilon b]$ and $\left[\epsilon^{\prime} a, \epsilon^{\prime} b\right]$ are not crossed by any periodic orbit. This ensures that the continuation morphism on Floer homology is bijective [31, Prop. 1.1]. 
Lemma 7.15. Let $\nu \in \mathbb{N}$ be fixed. For any $0<\epsilon<\epsilon^{\prime} \leq \epsilon_{\nu}$, there are chain equivalences of filtered chain complexes

$$
F C_{[\epsilon a, \epsilon b]}^{*}\left(Y_{\nu}^{\epsilon}, J_{\nu}\right) \stackrel{\psi_{\nu}^{\epsilon^{\prime}, \epsilon}}{\longrightarrow} F C_{\left[\epsilon^{\prime} a, \epsilon^{\prime} b\right]}^{*}\left(Y_{\nu}^{\epsilon^{\prime}}, J_{\nu}\right),
$$

which induce in homology the isomorphisms $\psi_{\nu}^{\epsilon^{\prime}, \epsilon}$ of Lemma 7.14.

Proof. Let us consider a homotopy of pseudo-gradient vector fields and symplectic forms $\left(Y_{\nu}^{\epsilon(s)}, \omega_{\epsilon(s)}\right), s \in \mathbb{R}$, between $\left(Y_{\nu}^{\epsilon}, \omega_{\epsilon}\right)$ and $\left(Y_{\nu}^{\epsilon^{\prime}}, \omega_{\epsilon^{\prime}}\right)$. The induced chain map is given by a count of solutions of the parametrized Floer equation $u_{s}+J_{s} u_{t}=Y_{\nu}^{\epsilon(s)}(t, u(s, t))$ for some split almost complex structure $J_{s}=\widetilde{J}_{B} \oplus J_{s}^{\mathrm{vert}}$. The projection $v=\pi \circ u$ of such a solution solves an equation of the form

$$
v_{s}+J_{B} v_{t}=\epsilon(s) J_{B} X_{f} .
$$

Since the almost complex structure $J_{B}$ can be chosen to be regular for (7.10), the induced chain map preserves the filtration. Note that solutions of (7.10) are actually reparametrized negative gradient trajectories of $f$. That the induced morphism in homology coincides with the map $\psi_{\nu}^{\epsilon^{\prime}, \epsilon}$ of Lemma 7.14 is the usual directed simple system property of Floer homology.

The chain morphism $\psi_{\nu}^{\epsilon^{\prime}, \epsilon}$ admits an inverse up to chain homotopy obtained by considering the reversed homotopy from $\left(Y_{\nu}^{\epsilon^{\prime}}, \omega_{\epsilon^{\prime}}\right)$ to $\left(Y_{\nu}^{\epsilon}, \omega_{\epsilon}\right)$, and is therefore a chain equivalence.

Lemma 7.16. Let $\nu \leq \nu^{\prime}$, so that $\epsilon_{\nu^{\prime}}<\epsilon_{\nu}$. For any $0<\epsilon \leq \epsilon_{\nu^{\prime}}$, we have monotonicity morphisms

$$
F C_{[\epsilon a, \epsilon b]}^{*}\left(Y_{\nu^{\prime}}^{\epsilon}, J_{\nu^{\prime}}\right) \stackrel{\sigma_{\nu, \nu^{\prime}}^{\epsilon}}{\longrightarrow} F C_{[\epsilon a, \epsilon b]}^{*}\left(Y_{\nu}^{\epsilon}, J_{\nu}\right)
$$

which preserve the filtrations.

Proof. We recall that

$$
Y_{\nu}^{\epsilon}=J_{\nu}\left(X_{\epsilon h_{\nu}}^{\epsilon}+\widetilde{X_{\epsilon c_{\nu} f}}+\left(X_{\epsilon G_{\nu}}^{\epsilon}\right)^{\mathrm{v}}\right), \quad Y_{\nu^{\prime}}^{\epsilon}=J_{\nu^{\prime}}\left(X_{\epsilon h_{\nu^{\prime}}}^{\epsilon}+\widetilde{X_{\epsilon c_{\nu^{\prime}} f}}+\left(X_{\epsilon G_{\nu^{\prime}}}^{\epsilon}\right)^{\mathrm{v}}\right) \text {. }
$$

The key point is to deform $Y_{\nu}^{\epsilon}$ to $Y_{\nu^{\prime}}^{\epsilon}$ through a deformation which is "good" in the sense of Definition 4.20. This will be achieved by catenating three good deformations obtained from the following data:

- a linear interpolation between $Y_{\nu}^{\epsilon}$ and $J_{\nu} X_{\epsilon h_{\nu}}^{\epsilon}$;

- an increasing homotopy $h(s, S)$ between $h_{\nu}$ and $h_{\nu^{\prime}}$ through convex Hamiltonians which are linear of slope $\lambda_{\max }(s)$ for $S \geq 1$;

- a linear interpolation between $J_{\nu^{\prime}} X_{\epsilon h_{\nu^{\prime}}}^{\epsilon}$ and $Y_{\nu^{\prime}}^{\epsilon}$.

First, each of the two linear interpolations defines a strong pseudogradient for each value of the deforming parameter $s \in \mathbb{R}$ and, moreover, the strong pseudo-gradient inequality is satisfied with a uniform constant 
by Proposition 5.8. As a consequence, the two interpolations define good deformations in the sense of Definition 4.20. Secondly, any choice of interpolation between $J_{\nu}$ and $J_{\nu^{\prime}}$ defines a genuine gradient deformation from $\nabla^{J_{\nu}} h_{\nu}$ to $\nabla^{J_{\nu^{\prime}}} h_{\nu^{\prime}}$, and in particular a good deformation in the sense of Definition 4.20, with uniform constant equal to 1 in the pseudo-gradient inequality. By catenation, we obtain a good deformation from $Y_{\nu}^{\epsilon}$ to $Y_{\nu^{\prime}}^{\epsilon}$, which moreover satisfies the strong pseudo-gradient inequality with a uniform constant.

Arguing as in Section 6, one can show that transversality can be achieved by a generic choice of homotopies of vertical almost complex structures and time-dependent horizontal distributions. The trajectories of the Floer equation on $\widehat{E}$ project on solutions of the equation

$$
v_{s}+J_{B} v_{t}=\epsilon c(s) J_{B} X_{f}
$$

on the base, for some smooth function $c: \mathbb{R} \rightarrow[0, \infty[$ which is nonzero near $\pm \infty$. Since the almost complex structure $J_{B}$ can be chosen to be regular for (7.12), the corresponding monotonicity morphisms preserve the filtrations. Note that solutions of (7.12) are actually reparametrized negative gradient lines of $f$.

Lemma 7.17. (a) Let $\nu \in \mathbb{N}$ and $q \in \mathbb{Z}$ be fixed. For any $0<\epsilon<\epsilon^{\prime} \leq \epsilon_{\nu}$, the filtered chain equivalences $\psi_{\nu}^{\epsilon^{\prime}, \epsilon}$ induce natural isomorphisms of local systems

$$
\mathcal{F} \mathcal{H}_{[\epsilon a, \epsilon b]}^{q}(\epsilon, \nu) \stackrel{\psi_{\nu}^{\epsilon^{\prime}, \epsilon}}{\longrightarrow} \mathcal{F} \mathcal{H}_{\left[\epsilon^{\prime} a, \epsilon^{\prime} b\right]}^{q}\left(\epsilon^{\prime}, \nu\right) .
$$

(b) Let $q \in \mathbb{Z}$ and $\nu \leq \nu^{\prime}$, so that $\epsilon_{\nu^{\prime}}<\epsilon_{\nu}$. For any $0<\epsilon \leq \epsilon_{\nu^{\prime}}$, the monotonicity morphisms $\sigma_{\nu, \nu^{\prime}}^{\epsilon}$ induce morphisms of local systems

$$
\mathcal{F} \mathcal{H}_{[\epsilon a, \epsilon b]}^{q}\left(\epsilon, \nu^{\prime}\right) \stackrel{\sigma_{\nu, \nu^{\prime}}^{\epsilon}}{\longrightarrow} \mathcal{F} \mathcal{H}_{[\epsilon a, \epsilon b]}^{q}(\epsilon, \nu) .
$$

Proof. (a) The filtered chain equivalence $\psi_{\nu}^{\epsilon^{\prime}, \epsilon}$ of Lemma 7.15 induces an isomorphism of spectral sequences

$$
\psi_{r}: E_{r}^{p, q}(\epsilon, \nu, a, b) \rightarrow E_{r}^{p, q}\left(\epsilon^{\prime}, \nu, a, b\right), \quad r \geq 1 .
$$

The terms $E_{1}$ are direct sums of stalks of the corresponding local systems, and we need to show that parallel transport commutes with $\psi_{1}$. We recall from Lemma 7.10 that, in a suitable trivialization, the parallel transport map is induced by a deformation of the Floer equation. On the other hand, it follows from the proof of Lemma 7.15 that the isomorphism $\psi_{1}$ is induced by deforming the Floer equation in each fiber $\widehat{E}_{p}, p \in \operatorname{Crit}(f)$. The compositions $\Phi\left(\epsilon^{\prime}, \nu\right) \circ \psi_{1}$ and $\psi_{1} \circ \Phi(\epsilon, \nu)$ are therefore induced by two deformations 
of the Floer equation having the same endpoints, and as such coincide at the level of homology.

(b) Let us denote by

$$
\sigma_{r}: E_{r}^{p, q}\left(\epsilon, \nu^{\prime}, a, b\right) \rightarrow E_{r}^{p, q}(\epsilon, \nu, a, b), \quad r \geq 1
$$

the map of spectral sequences induced by $\sigma_{\nu, \nu^{\prime}}^{\epsilon}$. We need to show that

$$
\Phi(\epsilon, \nu) \circ \sigma_{1}=\sigma_{1} \circ \Phi\left(\epsilon^{\prime}, \nu\right),
$$

and the argument is entirely similar to the one given at (a). The only difference is that $\sigma_{1}$ is not an isomorphism anymore, and this is reflected in the weaker statement we need to prove, namely the existence of a morphism of local systems, rather than the existence of an isomorphism.

Remark 7.18 (Floer local system). As a consequence of Lemma 7.17, we define the Floer local system

$$
\mathcal{F} \mathcal{H}_{[a, b]}^{q}(\widehat{F})=\lim _{\longleftarrow} \mathcal{F} \mathcal{H}_{\left[\epsilon_{\nu} a, \epsilon_{\nu} b\right]}^{q}\left(\epsilon_{\nu}, \nu\right)
$$

where the inverse limit is considered with respect to the maps

$$
\mathcal{F} \mathcal{H}_{\left[\epsilon_{\nu^{\prime}} a, \epsilon_{\nu^{\prime}} b\right]}^{q}\left(\epsilon_{\nu^{\prime}}, \nu^{\prime}\right) \stackrel{\psi_{\nu}^{\epsilon_{\nu}, \epsilon_{\nu^{\prime}}} \circ \sigma_{\nu^{\prime}, \nu}^{\epsilon_{\nu^{\prime}}} \longrightarrow}{\longrightarrow} \mathcal{F} \mathcal{H}_{\left[\epsilon_{\nu} a, \epsilon_{\nu} b\right]}^{q}\left(\epsilon_{\nu}, \nu\right), \quad \nu \leq \nu^{\prime} .
$$

Proof of Theorem A. We first show the existence of the requested spectral sequence. Let us consider the following diagram of chain complexes:

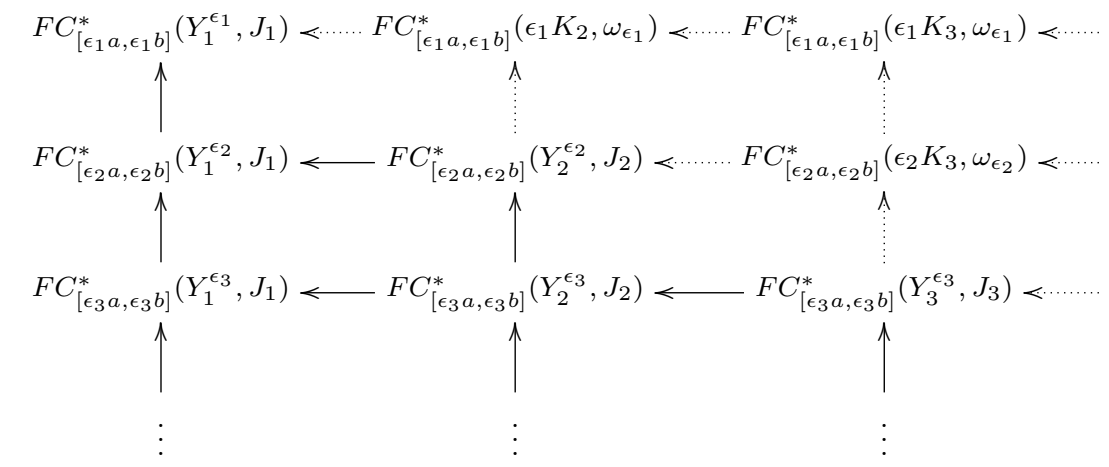

The vertical arrows induce in cohomology the isomorphisms $\psi_{\nu}^{\epsilon^{\prime}, \epsilon}, 0<\epsilon<$ $\epsilon^{\prime} \leq \epsilon_{0}$, of Lemmas 7.14 and 7.15. Moreover, the ones drawn with continuous lines preserve the filtrations given by (7.4).

The horizontal arrows are given by monotonicity morphisms, and the ones drawn with continuous lines preserve the filtrations by Lemma 7.16.

By naturality of the continuation and monotonicity morphisms in Floer homology, the above diagram commutes up to chain homotopy, and therefore commutes in homology. 
Let us denote

$$
F C^{*}(\nu, a, b)=F C_{\left[\epsilon_{\nu} a, \epsilon_{\nu} b\right]}^{*}\left(Y_{\nu}^{\epsilon_{\nu}}, J_{\nu}\right)
$$

According to the diagram there are filtered chain morphisms

$$
F C^{*}(\nu, a, b) \longleftarrow F C^{*}\left(\nu^{\prime}, a, b\right), \quad \nu \leq \nu^{\prime}
$$

which induce morphisms between the associated spectral sequences (7.5)

$$
E_{r}^{p, q}(\nu, a, b) \longleftarrow E_{r}^{p, q}\left(\nu^{\prime}, a, b\right) .
$$

We claim that

$$
E_{r}^{p, q}(a, b)=\lim _{\longleftarrow} E_{r}^{p, q}(\nu, a, b)
$$

is a spectral sequence which converges to $F H_{\left[\epsilon_{0} a, \epsilon_{0} b\right]}^{*}\left(\omega_{\epsilon_{0}}\right)$.

In order to show that $E_{r}^{p, q}(a, b)$ is a spectral sequence, we need to show that every exact sequence

$$
0 \longrightarrow \operatorname{im} d_{r}^{p-r, q+r-1}(\nu) \longrightarrow \operatorname{ker} d_{r}^{p, q}(\nu) \longrightarrow E_{r+1}^{p, q}(\nu) \longrightarrow 0
$$

remains exact in the inverse limit. This is the point where we need to use field coefficients. In this situation, the inverse limit is an exact functor if all terms involved in the inverse systems are finite dimensional vector spaces [8, Theorem VIII,5.7], which is the case in our setting.

The limit of the spectral sequence $E_{r}^{p, q}(a, b)$ is $\underset{\longleftarrow}{\lim _{\nu}} F H_{\left[\epsilon_{\nu} a, \epsilon_{\nu} b\right]}^{*}\left(Y_{\nu}^{\epsilon_{\nu}}, J_{\nu}\right)$ by definition. Since the vertical arrows in the diagram induce canonical isomorphisms in homology, we obtain canonical isomorphisms

$$
F H_{\left[\epsilon_{\nu} a, \epsilon_{\nu} b\right]}^{*}\left(Y_{\nu}^{\epsilon_{\nu}}, J_{\nu}\right) \simeq F H_{\left[\epsilon_{1} a, \epsilon_{1} b\right]}^{*}\left(\epsilon_{1} K_{\nu}, \omega_{\epsilon_{1}}\right)
$$

and therefore

$$
\lim _{\nu} F H_{\left[\epsilon_{\nu} a, \epsilon_{\nu} b\right]}^{*}\left(Y_{\nu}^{\epsilon_{\nu}}, J_{\nu}\right)=\lim _{\nu} F H_{\left[\epsilon_{1} a, \epsilon_{1} b\right]}^{*}\left(\epsilon_{1} K_{\nu}, \omega_{\epsilon_{1}}\right)=F H_{\left[\epsilon_{1} a, \epsilon_{1} b\right]}^{*}\left(\omega_{\epsilon_{1}}\right)
$$

The last equality holds by definition. On the other hand, we have $F H_{\left[\epsilon_{1} a, \epsilon_{1} b\right]}^{*}$ $\left(\omega_{\epsilon_{1}}\right) \simeq F H_{\left[\epsilon_{0} a, \epsilon_{0} b\right]}^{*}\left(\omega_{\epsilon_{0}}\right)$ by the deformation invariance Theorem 4.24, and the claim is proved.

The spectral sequences $E_{r}^{p, q}(a, b)$ are functorial with respect to the truncation of the action morphisms since all the intermediate complexes and maps involved in the construction have this property.

The definition of $E_{2}^{p, q}(a, b)$ and that of $\mathcal{F H}_{[a, b]}^{q}(\widehat{F})$ given in Remark 7.18 directly imply that

$$
E_{2}^{p, q}(a, b)=H^{p}\left(B ; \mathcal{F} \mathcal{H}^{q}(\widehat{F})\right) .
$$

The last assertion of the theorem states that the spectral sequence $E_{r}^{p, q}(a, b), r \geq 2$ and the local system $\mathcal{F H}_{[a, b]}^{q}(\widehat{F})$ are canonical, i.e., they do not depend on the various choices involved (the almost complex structures, 
the horizontal distribution used to lift $X_{f}$, the function $f$, the perturbations $G$, etc.) This is proved by the continuation method in Floer homology, i.e., by considering suitable $s$-dependent interpolating families. The key point is that all constructions can be performed so that the resulting morphisms preserve the filtrations, thus inducing isomorphisms between the corresponding spectral sequences. A glimpse of this phenomenon has already appeared in Lemmas 7.15 and 7.16 and we omit further details.

In the proof that follows the notation $0^{-}, 0^{+}$stands for a small enough negative, respectively, positive number.

Proof of Theorem B. Let us recall from Section 5.2 that, given $\nu \in \mathbb{N}$, the Hamiltonian $K_{\nu}$ and the vector field $Y_{\nu}^{\epsilon}$ are of the form

$$
K_{\nu}=h_{\nu}+c_{\nu} \tilde{f}+\varphi_{\nu}+G_{\nu}, \quad Y_{\nu}^{\epsilon}=J_{\nu}\left(X_{h_{\nu}}+\epsilon c_{\nu} \widetilde{X_{f}}+X_{\varphi_{\nu}}^{\mathrm{v}}+X_{G_{\nu}}^{\mathrm{v}}\right),
$$

where $\varphi_{\nu}$ is a $C^{2}$-small time-independent perturbation of $h_{\nu}+c_{\nu} \tilde{f}$ supported in $E \backslash\left\{1-\frac{\delta}{4} \leq S \leq 1\right\}$ for some $\delta>0$, and $G_{\nu}$ is a small time-dependent perturbation supported in an arbitrarily small neighborhood of $\partial E$. We use the notation $\widetilde{K}_{\nu}=h_{\nu}+c_{\nu} \widetilde{f}+\varphi_{\nu}$ and $E_{1-(\delta / 4)}=E \backslash\left\{1-\frac{\delta}{4} \leq S \leq 1\right\}$.

Let us choose $\mu>0$ smaller than $\min (\operatorname{Spec}(\partial E))$. A deformation argument shows that, as $\nu \in \mathbb{N}$ varies, the spectral sequences $E_{r}^{p, q}\left(\nu, 0^{-}, \mu\right)$ are canonically isomorphic at the page $r=1$, and therefore at all pages $r \geq 1$. It is thus enough to prove that $E_{r}^{p, q}\left(\nu, 0^{-}, \mu\right)$ is canonically isomorphic to the Leray-Serre spectral sequence under the assumption that the maximal slope of $h_{\nu}$ is smaller than $\min (\operatorname{Spec}(\partial E))$. This implies both assertions of Theorem B, namely the isomorphism of local systems $\mathcal{F H}_{\left[0^{-}, 0^{+}\right]}^{q}(\widehat{F}) \simeq \mathcal{H}^{k+q}(F, \partial F)$ and the isomorphism of spectral sequences $E_{r}^{p, q}\left(0^{-}, 0^{+}\right) \simeq{ }_{\mathrm{LS}} E_{r}^{n+p, k+q}, r \geq 2$.

We claim that, for a choice of $\nu$ as above, the complex $F C_{\left[0^{-}, \epsilon \mu\right]}^{*}(\epsilon, \nu)$ coincides with the Morse complex of $Y_{\nu}^{\epsilon}$ for $0<\epsilon \leq \epsilon_{\nu}$ if $c_{\nu},\left|\varphi_{\nu}\right|$ and $\left|G_{\nu}\right|$ are small enough. Note that $Y_{\nu}^{\epsilon}$ is a pseudo-gradient for the Morse function $\widetilde{K}_{\nu}$ on $E$. To prove the claim, we must show that Floer trajectories connecting critical points of $\widetilde{K}_{\nu}$ are independent of time. Since the Hamiltonian $\widetilde{K}_{\nu}$ is $C^{2}$-small on $E_{1-(\delta / 4)}$ and $E$ is symplectically aspherical, it is enough to show that these Floer trajectories are contained in $E_{1-(\delta / 4)}$. Let us argue by contradiction and assume that this is false. Then we find an $\epsilon>0$, sequences $c_{n} \rightarrow 0,\left|\varphi_{n}\right| \rightarrow 0,\left|G_{n}\right| \rightarrow 0$ and $t_{n} \in \mathbb{S}^{1}, n \geq 1$, as well as a sequence $u_{n}$ of maps solving the equation $\partial_{s} u_{n}+J_{\nu} \partial_{t} u_{n}=Y_{n}^{\epsilon} \circ u_{n}$ for

$$
Y_{n}^{\epsilon}=J_{\nu}\left(X_{h_{\nu}}+\epsilon c_{n} \widetilde{X_{f}}+X_{\varphi_{n}}^{\mathrm{v}}+X_{G_{n}}^{\mathrm{v}}\right) \text {, }
$$

satisfying $u_{n}(s, \cdot) \rightarrow x^{ \pm}, s \rightarrow \pm \infty$, for some distinct points $x^{ \pm} \in \operatorname{Crit}\left(\widetilde{K}_{\nu}\right)$, as well as

$$
u_{n}\left(0, t_{n}\right) \notin E_{1-(\delta / 4)}
$$


The uniform pseudo-gradient property for $\mathcal{Y}_{n}^{\epsilon}=J_{\nu} \frac{d}{d t}-Y_{n}^{\epsilon}$ provides a uniform bound on the energy $E\left(u_{n}\right)$ for all $n$, whereas a uniform $C^{0}$-bound on the sequence $u_{n}$ follows from Theorem 4.6. By Floer-Gromov compactness, we obtain in the limit a nonconstant map $u$ satisfying $u_{t}+J_{\nu} u_{s}=J_{\nu} X_{h_{\nu}}$ and $u(s, \cdot) \rightarrow y^{ \pm}$for two distinct points $y^{ \pm} \in E_{1-(\delta / 4)}$, and such that $u(0, t) \notin E_{1-(\delta / 4)}$ for some $t \in \mathbb{S}^{1}$. Since $h_{\nu}=h_{\nu}(S)$, we can apply the maximum principle as in [31, Lemma 1.8] and show that $u$ cannot have a local maximum in $\partial E \times[1-(\delta / 4), \infty[$ (see also Remark 4.17). The map $u$ must therefore be entirely contained in $E_{1-(\delta / 4)}$, a contradiction.

Using the notation $E_{r}^{p, q}(\nu, a, b)$ for the spectral sequence associated to the filtered complex $F C_{\left[\epsilon_{\nu} a, \epsilon_{\nu} b\right]}^{*}\left(Y_{\nu}^{\epsilon_{\nu}}, J_{\nu}\right)$, we must prove that we have a canonical isomorphism of spectral sequences

$$
E_{r}^{p, q}\left(\nu, 0^{-}, \mu\right) \simeq_{\mathrm{LS}} E_{r}^{n+p, k+q} .
$$

At this point, we need to recall the construction of the Leray-Serre spectral sequence using cellular homology, as defined in Section 7.2.1. Assume one has $\mathrm{CW}$-decompositions of $E$ and $B$ with the property that the projection of a cell $e_{i}^{k}$ in $E$ is a cell $\pi\left(e_{i}^{k}\right)$ in $B$. The cellular complex

$$
\operatorname{Cell}^{k}(E)=\bigoplus_{i} \mathbb{Z}\left\langle e_{i}^{k}\right\rangle
$$

is then naturally filtered by

$$
F_{p} \operatorname{Cell}^{k}(E)=\bigoplus_{\operatorname{dim} \pi\left(e_{i}^{k}\right) \geq p} \mathbb{Z}\left\langle e_{i}^{k}\right\rangle,
$$

and the spectral sequence associated to this filtration is ${ }_{\mathrm{LS}} E_{r}^{p, q}$. This is precisely the definition given in [21] tailored to the setup of cellular cohomology.

The isomorphism (7.13) follows now from the fact that the underlying filtered complexes are isomorphic. Indeed, the complex $F C_{\left[\epsilon_{\nu} a, \epsilon_{\nu} b\right]}^{*}\left(Y_{\nu}^{\epsilon_{\nu}}, J_{\nu}\right)$ was shown to coincide with the Morse complex, the latter is tautologically identified with the cellular complex on $E$ by our standing assumption on the behavior of $K_{\nu}$ near its critical points (cf. Section 7.3), and the filtration (7.4) on the Morse complex tautologically coincides with the filtration (7.14) on the cellular complex due to our standing assumption on the behavior of $f$ near its critical points. The shift in the grading comes from the fact that the Floer complex is graded by minus the Conley-Zehnder index instead of the Morse index.

\section{Appendix A. On Symplectic Forms and the Taming Property}

Proposition A.1. Let $\omega_{1}, \omega_{2}$ be symplectic forms on a manifold B. Let $\mathcal{J}\left(B, \omega_{i}\right) \subset \mathcal{J}_{\tau}\left(B, \omega_{i}\right)$ be the contractible sets of almost complex structures which are compatible with, respectively tamed by $\omega_{i}, i=1,2$. 
Assume $\mathcal{J}\left(B, \omega_{1}\right) \subset \mathcal{J}_{\tau}\left(B, \omega_{2}\right)$. Then $\omega_{1}=f \omega_{2}$ for some strictly positive function $f$ on $B$, and in particular $\mathcal{J}\left(B, \omega_{1}\right)=\mathcal{J}\left(B, \omega_{2}\right), \mathcal{J}_{\tau}\left(B, \omega_{1}\right)=$ $\mathcal{J}_{\tau}\left(B, \omega_{2}\right)$. If $\operatorname{dim} B \geq 4$, then $f$ is constant on every component of $B$.

Remark A.2. The assumption $\mathcal{J}\left(B, \omega_{1}\right) \subset \mathcal{J}_{\tau}\left(B, \omega_{2}\right)$ is satisfied for example if $\mathcal{J}\left(B, \omega_{1}\right) \subset \mathcal{J}\left(B, \omega_{2}\right)$, or $\mathcal{J}_{\tau}\left(B, \omega_{1}\right) \subset \mathcal{J}_{\tau}\left(B, \omega_{2}\right)$.

Proof. We can work fiberwise in each tangent space and, without loss of generality, assume that $\omega_{1}$ and $\omega_{2}$ are linear symplectic forms in $\mathbb{R}^{2 n}$. We denote $\mathcal{J}\left(\omega_{i}\right)=\mathcal{J}\left(\mathbb{R}^{2 n}, \omega_{i}\right), i=1,2$. The statement is obvious for $n=1$ and we assume $n \geq 2$. Fix $v, w \in \mathbb{R}^{2 n}$ which are noncollinear. We claim that $\omega_{1}(v, w)=0$ if and only if $\omega_{2}(v, w)=0$. Assume first $\omega_{1}(v, w)=0$. There exists $J \in \mathcal{J}\left(\omega_{1}\right)$ such that $V=\operatorname{Sp}\langle v, J v\rangle$ and $W=\operatorname{Sp}\langle w, J w\rangle$ are orthogonal with respect to $\omega_{1}$. Let $T=\operatorname{Sp}\langle v, J v, w, J w\rangle$. By hypothesis, the space $V$ is symplectic for $\omega_{2}$ and its orthogonal $V^{\perp_{\omega_{2}}}$ in $T$, denoted $V^{\prime}$, is a complement of $V$ and therefore generated by $w+a v+b J v$ and $J w+c v+d J v$, where $a, b, c, d \in \mathbb{R}$ are suitable constants. We show that $a=b=c=d=0$. Assume for example $d \neq 0$. We have $\omega_{1}(v, J w+c v+d J v)=d \cdot \omega_{1}(v, J v) \neq 0$. There exists therefore $J^{\prime} \in \mathcal{J}\left(\omega_{1}\right)$ such that $J w+c v+d J v=\operatorname{sign}(d) \cdot J^{\prime} v$. Then $\omega_{2}(v, J w+c v+d J v)=\operatorname{sign}(d) \cdot \omega_{2}\left(v, J^{\prime} v\right) \neq 0$, which contradicts the definition of $V^{\prime}$. One proves in the same way that $a=b=c=0$. Assume now that $\omega_{2}(v, w)=0$. If $\omega_{1}(v, w) \neq 0$, there exists $J \in \mathcal{J}\left(\omega_{1}\right)$ such that $w=$ $\operatorname{sign}\left(\omega_{1}(v, w)\right) \cdot J v$, hence $\omega_{2}(v, w)= \pm \omega_{2}(v, J v) \neq 0$, a contradiction. One can easily see now that we also have $\omega_{1}(v, w)>0$ if and only if $\omega_{2}(v, w)>0$.

Let now $e_{1}, f_{1}, \ldots, e_{n}, f_{n}$ be a symplectic basis for $\omega_{1}$, i.e., $\omega_{1}=\sum_{i=1}^{n} e_{i}^{*} \wedge$ $f_{i}^{*}$. The above implies that $\omega_{2}=\sum_{i=1}^{n} \lambda_{i} e_{i}^{*} \wedge f_{i}^{*}, \lambda_{i}>0$. We show that all the $\lambda_{i}$ are equal: for $1 \leq i, j \leq n$, we have $\omega_{1}\left(e_{i}+e_{j}, f_{i}-f_{j}\right)=0$, hence $0=\omega_{2}\left(e_{i}+e_{j}, f_{i}-f_{j}\right)=\lambda_{i}-\lambda_{j}$. Therefore, $\omega_{1}=\lambda \omega_{2}, \lambda>0$ and $\mathcal{J}\left(\omega_{1}\right)=\mathcal{J}\left(\omega_{2}\right), \mathcal{J}_{\tau}\left(\omega_{1}\right)=\mathcal{J}_{\tau}\left(\omega_{2}\right)$.

We can now write $\omega_{1}=f \omega_{2}$, with $f: B \longrightarrow \mathbb{R}^{+}$smooth. Assume $\operatorname{dim} B \geq 4$. By closedness we get $d f \wedge \omega_{2}=0$. Assume $d f$ is not identically zero, i.e., there exist $x \in B$ and $X \in T_{x} B$ such that $d f_{x}(X)>0$. The germ of $\Sigma=f^{-1}(f(x))$ at $x$ is a smooth hypersurface of dimension $\geq 3$, on which $\lambda=\iota_{X} \omega_{2}$ is nonzero. The form $\omega_{2}$ is nondegenerate on ker $\lambda$ and this implies $\left(d f \wedge \omega_{2}\right)_{x} \neq 0$, a contradiction.

\section{References}

[1] R. Bott and L.W. Tu, Differential forms in algebraic topology, Vol. 82 Graduate Texts in Mathematics, Springer-Verlag, New York, 1982.

[2] F. Bourgeois and A. Oancea, A long exact sequence for contact and symplectic homology, 2007, arXiv:0704.2169.

[3] G.E. Bredon, Topology and geometry, Vol. 139, Graduate Texts in Mathematics, Springer-Verlag, New York, 1993. 
[4] K. Cieliebak, Handle attaching in symplectic homology and the chord conjecture, J. Eur. Math. Soc. (JEMS), 4(2) (2002), 115-142.

[5] — Subcritical Stein manifolds are split, 2002, arXiv:math/0204351.

[6] K. Cieliebak, A. Floer, and H. Hofer, Symplectic homology. II. A general construction, Math. Z. 218(1) (1995), 103-122.

[7] K. Cieliebak, A.R. Gaio, I. Mundet i Riera and D. Salamon, The symplectic vortex equations and invariants of Hamiltonian group actions. J. Symplectic Geom. 1(3) (2002), 543-645.

[8] S. Eilenberg and N. Steenrod, Foundations of algebraic topology, Princeton University Press, Princeton, NJ, 1952.

[9] A. Floer, Symplectic fixed points and holomorphic spheres, Comm. Math. Phys. 120(4) (1989), 575-611.

[10] A. Floer and H. Hofer, Coherent orientations for periodic orbit problems in symplectic geometry, Math. Z. 212(1) (1993), 13-38.

[11] - Symplectic homology. I. Open sets in $\boldsymbol{C}^{n}$, Math. Z. 215(1) (1994), 37-88.

[12] A. Floer, H. Hofer, and D. Salamon, Transversality in elliptic Morse theory for the symplectic action. Duke Math. J. 80(1) (1995), 251-292.

[13] A. Floer, H. Hofer, and C. Viterbo, The Weinstein conjecture in $P \times \mathbf{C}^{\ell}$, Math. Z., 203(3) (1990), 469-482.

[14] P. Gauduchon, Connexions linéaires, classes de Chern, théorème de Riemann-Roch, Holomorphic Curves in Symplectic Geometry, Vol. 117, Progr. Math. 113-162, Birkhäuser, Basel, 1994.

[15] P.A. Griffiths, Hermitian differential geometry, Chern classes, and positive vector bundles, Global Analysis (Papers in Honor of K. Kodaira), 185-251, Univ. Tokyo Press, Tokyo, 1969.

[16] V. Guillemin, E. Lerman and S. Sternberg, Symplectic fibrations and multiplicity diagrams, Cambridge University Press, Cambridge, 1996.

[17] H. Hofer and D. Salamon, Floer homology and Novikov rings, The Floer Memorial Volume, Vol. 133, Progr. Math. 483-524, Birkhäuser, Basel, 1995.

[18] M. Hutchings, Floer homology of families I, 2003, math.SG/0308115.

[19] S. Kobayashi, Differential geometry of complex vector bundles, Vol. 15, Publications of the Mathematical Society of Japan, Princeton University Press, Princeton, NJ, 1987, Kanô Memorial Lectures, 5.

[20] F. Laudenbach, On the Thom-Smale complex, An extension of a theorem by Cheeger and Müller by J.-M. Bismuth and W. Zhang, Vol. 205, Astérisque, 205-214, Soc. Math. France, 1992.

[21] J. McCleary, A user's guide to spectral sequences, Vol. 58, Cambridge Studies in Advanced Mathematics, Cambridge University Press, Cambridge, second edition, 2001.

[22] D. McDuff and D. Salamon, Introduction to symplectic topology, Oxford Mathematical Monographs, The Clarendon Press, Oxford University Press, New York, 1995. (Oxford Science Publications).

[23] $\longrightarrow$ J-holomorphic curves and symplectic topology, Vol. 52, American Mathematical Society Colloquium Publications, American Mathematical Society, Providence, RI, 2004. 
[24] J. Milnor and J. Stasheff, Characteristic classes, Vol. 76, Annals of Mathematics Studies, Princeton University Press, Princeton, NJ, 1974.

[25] A. Oancea, The Künneth formula in Floer homology for manifolds with restricted contact type boundary, Math. Ann. 334(1) (2006), 65-89.

[26] D. Salamon, Lectures on Floer homology, Symplectic geometry and topology (Park City, UT, 1997), Vol. 7, IAS/Park City Math. Ser. 143-229, Amer. Math. Soc. Providence, RI, 1999.

[27] D. Salamon and E. Zehnder, Morse theory for periodic solutions of Hamiltonian systems and the Maslov index, Comm. Pure Appl. Math. 45(10) (1992), 1303-1360.

[28] P. Seidel, $\pi_{1}$ of symplectic automorphism groups and invertibles in quantum homology rings, Geom. Funct. Anal. 7(6) (1997), 1046-1095.

[29] P. Seidel, A biased view of symplectic cohomology, 2007, arXiv:0704.2055.

[30] N.E. Steenrod, Homology with local coefficients, Ann. Math. 44(2) (1943), 610-627.

[31] C. Viterbo, Functors and computations in Floer homology with applications. I, Geom. Funct. Anal. 9(5) (1999), 985-1033.

[32] A. Weinstein, On the hypotheses of Rabinowitz' periodic orbit theorems, J. Diff. Equations 33(3) (1979), 353-358.

Université Louis Pasteur

Institut De Recherche Mathématique Avancée (IRMA)

F-67084 Strasbourg, France

E-mail address: oancea@math.u-strasbg.fr

Received 8/13/2007, accepted 3/21/2008

I am indebted to Claude Viterbo for having suggested the topic to me, for his confidence and for his constant enthusiastic support as a Ph.D. advisor. I am equally indebted to Dietmar Salamon, from whom I learnt that finding the right degree of generality for a problem represents half the way to its solution. Work on this paper has spread over a long period, during which the author was successively supported by Université Paris Sud, École Polytechnique (Palaiseau), École Normale Supérieure de Lyon, ETH Zürich, and Université Louis Pasteur, Strasbourg. 